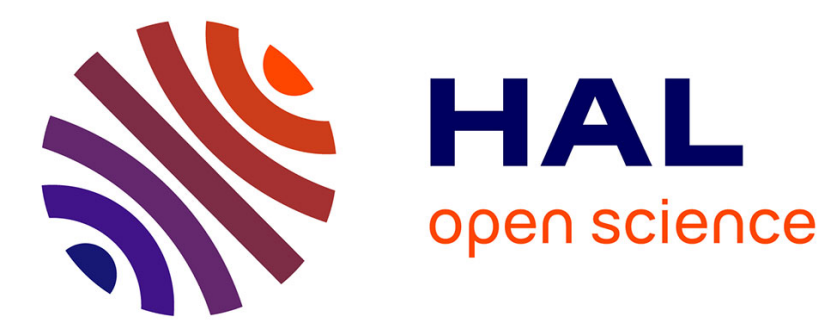

\title{
On nonlinear vibrations of systems with many degrees of freedom
}

\author{
Reinhardt Rosenberg
}

\section{To cite this version:}

Reinhardt Rosenberg. On nonlinear vibrations of systems with many degrees of freedom. Advances in Applied Mechanics, 1966, 10.1016/S0065-2156(08)70008-5 . hal-01544496

\section{HAL Id: hal-01544496 \\ https://hal.science/hal-01544496}

Submitted on 17 Jul 2017

HAL is a multi-disciplinary open access archive for the deposit and dissemination of scientific research documents, whether they are published or not. The documents may come from teaching and research institutions in France or abroad, or from public or private research centers.
L'archive ouverte pluridisciplinaire HAL, est destinée au dépôt et à la diffusion de documents scientifiques de niveau recherche, publiés ou non, émanant des établissements d'enseignement et de recherche français ou étrangers, des laboratoires publics ou privés. 


\title{
On Nonlinear Vibrations of Systems with Many Degrees of Freedom
}

\author{
By R. M. ROSENBERG
}

Department of Mechanical Engineering, Division of Applied Mechanics, University of California, Berkeley, California

\section{INTRODUCTION}

The study of the vibrations of nonlinear systems with many degrees of freedom is concerned with the search for some or all periodic solutions of systems of nonlinear differential equations, and to deduce as many properties of these solutions as the state of the applicable mathematical knowledge permits. Unfortunately, this body of knowledge is limited and not unified; in consequence, many and varied disciplines within mathematics are commonly used to deduce partial results. In a general way, these results fall into two broad categories: those which apply to systems that are "weakly nonlinear,"* and those which apply when the systems are "strongly nonlinear."

The results in the first category contain a good deal of detailed information, and they resemble in many ways those familiar from linear theory. Those in the latter category usually contain fewer details, being more concerned with general questions of existence, uniqueness, boundedness and stability of solutions.

The task of presenting a meaningful survey of the methods and results concerning the vibrations of nonlinear multi-degree-of-freedom systems is too ambitious for this relatively brief contribution. Moreover, several survey articles and books, foremost among them the recent, admirable work by Minorski $[\mathbf{1}]$ have served this purpose. Here, it is intended to display certain geometrical methods, and to summarize the results stemming from their application.

The only systems considered are those whose mechanical models can be constructed of masses and "massless" springs. One, several, or all masses may also be acted on by time-dependent, periodic "exciting forces." The nonlinearities of these systems are those arising from "nonlinear springs." Within these restrictions, the system may be linear, "weakly" or "strongly" nonlinear, or "nonlinearizable." In the geometrical methods used here, "weak" nonlinearity is of no advantage, and results for strongly nonlinear

\footnotetext{
- All terms in quotation marks are defined precisely in the text.
} 
or nonlinearizable systems are frequently as easy to deduce as those of almost linear systems.

It is plausible that general solutions (i.e. the class of all possible motions) cannot be deduced for such nonlinear systems. However, if the class of desired solutions is suitably restricted, one can frequently gain a great deal of detailed knowledge regarding them, no matter how strongly nonlinear the system. However, to be meaningful for the physical scientist, this restricted class of desired solutions must be such as to explain and/or predict the incidence of physically important phenomena. In linear systems, these physically interesting solutions are the so-called "natural free vibrations," and the steady-state forced vibrations. It is precisely these types of motion that are treated here for nonlinear systems.

The methods used to deduce these motions are not conventional (in the sense of conventional present-day research in nonlinear vibrations), but they are classical in the sense of theoretical mechanics that originated with Hamilton, Jacobi and their contemporaries and that was described and extended relatively recently in a beautiful paper by Synge [2]. These methods are especially useful in the search for "free vibrations in normal modes" when the systems are strongly nonlinear. However, the concept of "normal modes" and of eigenvalues is well defined only in linear systems. In fact, the demonstration of the existence of eigenvalues has its roots in the theory of quadratic forms, and its application to vibration problems requires that the potential energy be a quadratic form. But, it is the very essence of nonlinearity, as defined here, that the potential energy is not a quadratic form. Hence, the question of existence of normal-mode vibrations cannot be decided in the conventional way of finding eigenvalues, and of attaching to each an eigenfunction. Therefore, new definitions and methods are required when the problems are nonlinear.

The usefulness of the concept of normal-mode vibration is, of course, greatly impaired in nonlinear problems because the principle of superposition fails. Nevertheless, the normal-mode vibration retains, even in nonlinear systems, a position of central importance because, as will be shown, resonance occurs in the neighborhood of normal-mode vibrations, whether the system is linear or nonlinear. Hence, when the amplitude of the forcing function is small, the solution of the normal-mode vibration problem furnishes the "zero'th order" solution of the problem of steady-state forced vibration, as demonstrated by Rauscher [3], for instance. As in the Duffing problem [4], it is also necessary in problems with many degrees of freedom to examine the stability of the solutions; this is done in the last section.

Although examples are given, the question of application has not been treated in a general way. Suffice it to say that in our model a displacement of any one mass has "an influence in depth"; i.e., its effect is not only felt by the neighboring masses, but by all of them. Hence, it is likely that the 
systems discussed here are applicable to crystal and molecule vibrations. It is also quite possible that one can discuss, by the methods described here, the mechanism of pulsating stars [5]. At any rate, these methods have been examined in detail [6] by an astronomer having this purpose in mind.

No attempt has been made to cite in the bibliography all recent literature dealing with vibrations of nonlinear systems having many degrees of freedom; it is, in fact, restricted to the material which was used in this contribution.

\section{Admissible Systems and Motions}

\section{The General System}

The general system considered here may be modelled on a great variety of mechanical, electrical, or other devices, and its configuration may be determined by many different types of physical measurements. One such model

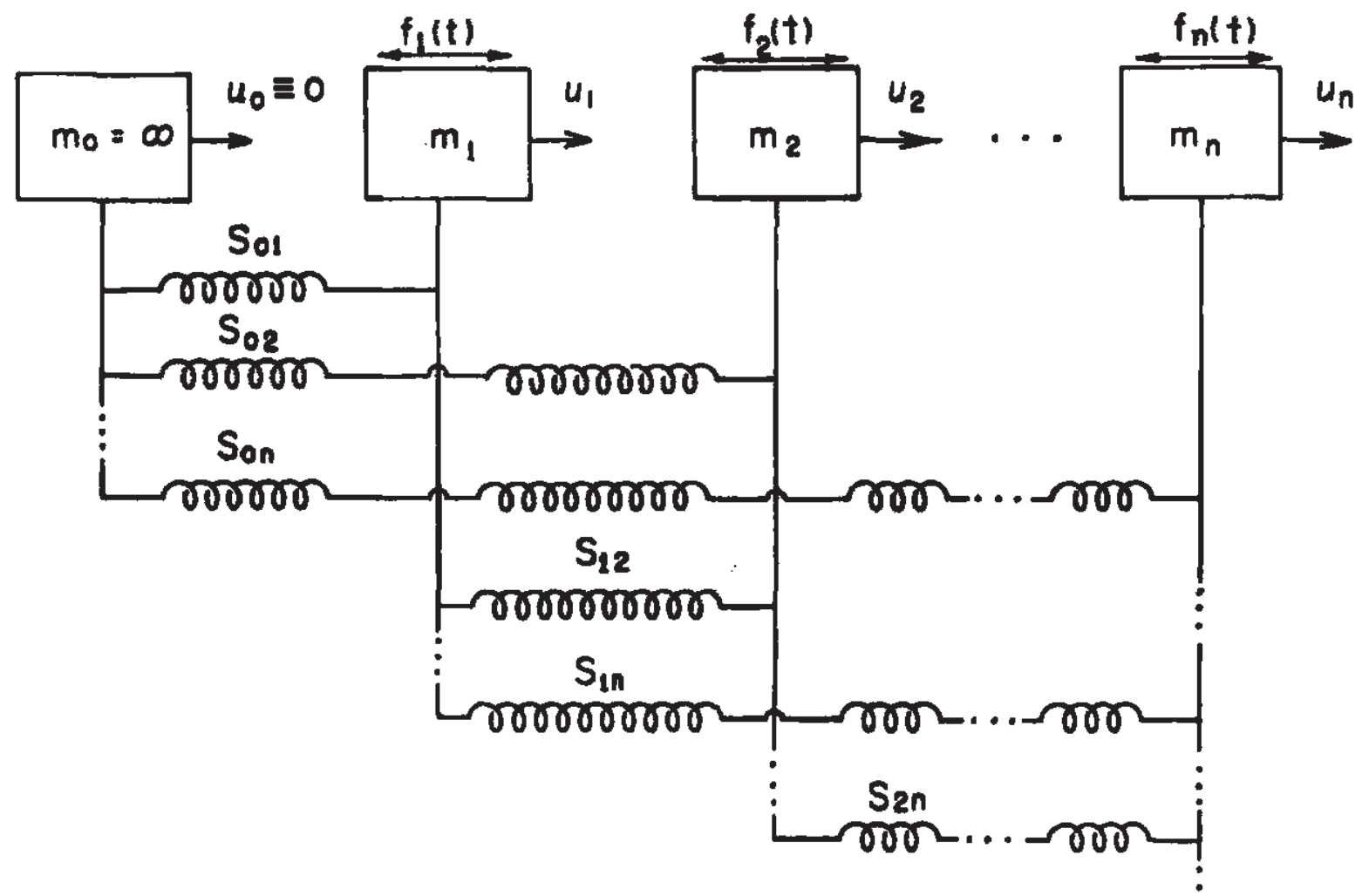

FIG. 1. Mechanical model of spring-mass system.

is Figure 1. It consists of a chain of $n+1$ mass points $P_{i},(i=0, \ldots, n)$ and of $s$ springs, $n \leqslant s \leqslant n(n+1) / 2$.

The mass of $P_{r}$ is $m_{r}\left(0<m_{r} \leqslant \infty\right)$. Any $m_{r}=\infty$ is a fixed point in inertial space. We require that at least one of the mass points have infinitely large mass, e.g. $m_{0}=\infty$.

The term "spring" is used to describe a massless one-dimensional device that changes its length under the action of a force. In physical terms, a spring is assumed to shorten under a compressive force of given magnitude 
precisely by the same amount as it lengthens under a tensile force of the same magnitude, and it is capable of storing, but not of creating or destroying energy. These conditions are fulfilled when

$$
\bar{O}_{i j}(w)=\bar{O}_{i j}(|w|)
$$

and when

$$
F_{i j}(w)=\frac{d}{d w} \hat{O}_{i j}(w)
$$

where $C_{i j}(w)$ is the negative of the potential energy $\nabla_{i j}$ stored in the spring $S_{i j}$ terminating on the $i$ th and $j$ th mass points, $w$ is the length change of $S_{i j}$, and $F_{i j}$ is the force which has produced the length change. Evidently, combining (2.1) and (2.2), one has

$$
F_{i j}(w)=-F_{i j}(-w) \text {. }
$$

Each mass point is connected to one, several, or all other mass points by a spring; hence, the maximum number of springs is $n(n+1) / 2$. If one extremity of a spring terminates on an infinitely large mass point it is called an anchor spring; all others are coupling springs. It is not essential that all $n(n+1) / 2$ springs be present in the system. However, it is required that, in the absence of additional constraints, no mass point can be displaced without giving rise to an elastic force acting on every mass point. For instance if

$$
\bar{O}_{i r} \equiv 0, \quad(i=0, \ldots, r-1),
$$

the $r$ th mass would be decoupled from all its neighbors on the left, and the system of $\dot{n}+1$ mass points would be separated into two spring-mass systems with no elastic coupling between them. Such a separation into several mutually uncoupled systems is excluded. In consequence the minimum number of springs is $n$.

Each mass point has a single degree of translational freedom $u_{i}$ in the direction of the chain. In view of $m_{0}=\infty$, one has $u_{0} \equiv 0$; hence, the system cannot accelerate in the rigid body mode.

Each mass point may be acted upon by a time-dependent force, called an exciting force and denoted by $f_{i}(t)$. Exciting forces act in the direction of the chain of mass points. It is assumed that the spring forces $F_{i j}$ and the exciting forces $\tilde{f}_{i}$ are the only forces acting on the mass points.

\section{Admissible Systems}

In order to fit into the framework of vibrations, linear or nonlinear, systems must have certain properties whose physical description is:

(i) in the absence of exciting forces, the system must possess a single equilibrium configuration, and it can execute "free vibrations" about that equilibrium configuration; 
(ii) in the presence of exciting forces, the system can execute so-called "steady-state forced vibrations."

Some of these conditions can be insured by endowing the potential function of the entire system with certain properties. This potential function is, evidently,

$$
0=\sum_{i=0}^{n-1} \sum_{i=i+1}^{n} O_{i j}\left(u_{i}-u_{i}\right)
$$

where $u_{j}-u_{i}$ is the length change in the spring $S_{i j}$.

We shall assume that, on any domain where it exists, $\bar{O}$ is smooth and its first partial derivatives with respect to the $u_{i}$ are, at least, piecewise smooth. The second partial derivatives of $\bar{O}$ are thus assumed to exist everywhere except possibly at isolated points of discontinuity of the $\partial \circlearrowright / \partial u_{i}$. These assumptions are consistent with the physical properties of springs in that they provide for the possibility of spring forces "with corners"; yet they assure the existence of unique solutions under specified initial conditions.

In addition, we require that the potential function $\bar{O}$ be negative definite, or

$$
\left\{\begin{array}{l}
O(0, \ldots, 0)=0 \\
O\left(u_{1}, \ldots, u_{n}\right)<0 \text { when the } u_{1}, \ldots, u_{n} \text { do not vanish simultaneously }
\end{array}\right.
$$

and the first partial derivatives of $\bar{U}$ vanish at the origin only, or

$$
\frac{\partial}{\partial u_{1}} O(0, \ldots, 0)=\ldots=\frac{\partial}{\partial u_{n}} \delta(0, \ldots, 0)=0
$$

Finally, as a consequence of (2.1) and (2.5), 0 is symmetric with respect to the origin of the $u_{1}, \ldots, u_{n}$-space, or

$$
O\left(u_{1}, \ldots, u_{n}\right)=O\left(-u_{1}, \ldots,-u_{n}\right) .
$$

Equations (2.6) and (2.7) insure that there exists a unique equilibrium configuration, and the origin of the $u_{i}$ has been so chosen that every $u_{i}$ vanishes in the equilibrium configuration. Equation (2.8) is a direct consequence of the symmetry properties of the individual springs, described in (2.3).

A potential function $\bar{O}$ which satisfies all of the above properties will be called admissible. Moreover, when $\bar{O}$ is admissible, the autonomous system is admissible. 
Exciting forces $f_{i}(t)$ are required to be at least piecewise continuous, and bounded for all $t$. In addition, they must satisfy

$$
f_{i}(t)=f_{i}(t+T), \quad(i=1, \ldots, n),
$$

as well as

$$
\frac{1}{T} \int_{0}^{T} f_{i}(\tau) d \tau=0,
$$

where $T$ is a constant. Equation (2.9) says that the exciting forces are periodic and, if there is more than one such force, they all have the same period $T$.

Exciting forces $f_{i}(t)$ which satisfy the above conditions are called admissible. Moreover, when both, $\bar{O}$ and the $\vec{f}_{i}(t)$ are admissible, the nonautonomous system is said to be admissible.

Among admissible systems, autonomous or not, we distinguish between those that are linearizable, nonlinearizable, and weakly, or strongly nonlinear. In our system, these properties are associated with the spring forces; hence, it may be best to define them in terms of these forces; i.e., in terms of the partial derivatives of $\bar{O}$.

Because of the admissibility conditions on $\bar{C}$, these derivatives may be written in the form

$$
\frac{\partial}{\partial u_{i}} \hat{O}=\sum_{j=1}^{n} \bar{a}_{i j} u_{j}+P_{i}\left(u_{1}, \ldots, u_{n}\right)
$$

where the functions $\tilde{P}_{i}$ vanish at the origin and are of degree higher than 1 and, because of $(2.8)$,

$$
\bar{a}_{i j}=\bar{a}_{j i} .
$$

Let the determinant of the $\bar{a}_{i j}$ be defined by

$$
\bar{a}=\left|\bar{a}_{i j}\right| .
$$

Then, a system is said to be linearizable if $\bar{a} \neq 0$, and nonlinearizable if $\bar{a}=0$. A system is said to be weakly nonlinear if

$$
\bar{P}_{i}\left(u_{1}, \ldots, u_{n}\right)=\varepsilon_{i} \bar{Q}_{i}\left(u_{1}, \ldots, u_{n}\right), \quad(i=1, \ldots, n)
$$

where every $\left|\varepsilon_{i}\right| \ll|\bar{a}|$ where $\bar{a}$ is defined in (2.13) and every $\bar{Q}_{i}$ is bounded. If (2.13) is not satisfied, the system is said to be strongly nonlinear. 


\section{Admissible Motions}

From the definition of admissible systems, it is evident that these may be strongly nonlinear, or even nonlinearizable. In consequence, it is not likely that one can find the general solutions of the equations of motion or, the class of all motions of which the system is capable. However, it is possible to deduce certain types of motion and, in particular, the motions which are the most important from the point of view of technical application. These are the motions which, in linear systems, are called the "natural free vibrations in normal modes" if the system is autonomous, and the "steadystate forced vibrations" if the system is nonautonomous. The latter include the phenomena of "resonance" and of the tuned dynamic vibration absorber.

Both, the free vibrations in normal modes, and the steady-state forced vibrations are so-called "vibrations-in-unison". An admissible system is said to execute a vibration-in-unison if its motion satisfies all of the following properties [7]:

(i) There exists a $T=$ constant such that

$$
u_{i}(t)=u_{i}(t+T), \quad(i=1, \ldots, n) .
$$

In words, the motions of all masses are equiperiodic.

(ii) If $t_{r}$ is any instant of time, there exists a single $t_{0}$ in

$$
t_{r} \leqslant t \leqslant t_{r}+T / 2
$$

such that

$$
u_{i}\left(t_{0}\right)=0, \quad(i=1, \ldots, n) .
$$

In words, during any time interval of a half period, the system passes precisely once through its equilibrium configuration.

(iii) There exists a single $t_{1} \neq t_{0}$ in

$$
t_{r} \leqslant t \leqslant t_{r}+T / 2
$$

such that

$$
\dot{u}_{i}\left(t_{1}\right)=0 .
$$

In words, during any time interval of a half period, the velocities of all masses vanish precisely once or, all masses attain their maximum displacement (in absolute value) precisely once during any interval of a half period.

(iv) Let $r$ (fixed) be any one of the $i=1, \ldots, n$. Then every $u_{i}(t)$ and $u_{r}(t)$ may be written, for all $t$, in the form

$$
u_{i}=u_{i}\left(u_{r}\right)
$$


in which $t$ is a parameter. It is now required that every $u_{i}\left(u_{r}\right)$ be singlevalued. In other words, the displacement of any one mass at any instant of time determines uniquely that of every other mass at the same instant of time.

These are well-known properties of normal-mode vibrations, and of steady-state forced vibrations, of the linear system. We shall show that these same types of motion also exist in admissible nonlinear systems. Any motion of an admissible system which satisfies (2.15) to (2.18) is said to be an admissible motion.

Among admissible motions, it is necessary to distinguish between those which are "similar" and those which are not. An admissible motion is said to be similar [8] if

$$
u_{j}(t) / u_{i}(t) \equiv c_{i j}=\text { const. }
$$

This terminology corresponds to common usage because, when $(2.19)$ is satisfied by an admissible motion, the wave-shapes of the time-displacement curves are all geometrically similar. When (2.19) is not satisfied by an admissible motion, that motion is said to be nonsimilar.

The normal-mode motions of linear systems, and their steady-state forced vibrations under simple harmonic excitation are of the form

$$
u_{i}=Q_{i} \cos \omega t, \quad(i=1, \ldots, n)
$$

where the $Q_{i}$ are constants. Hence, these motions satisfy (2.19); i.e., they are similar. We shall show that the linear system is not the only one having similar vibrations-in-unison. In fact, there exist strongly nonlinear, and nonlinearizable systems whose vibrations-in-unison are also similar.

\section{The Trajectories in Configuration Space}

\section{Transformation and Trajectories}

The equations of motion of the physical system are

$$
m_{i} \ddot{u}_{i}=\frac{\partial}{\partial u_{i}} \sigma\left(u_{1}, \ldots, u_{n}\right)+f_{i}(t), \quad(i=1, \ldots, n)
$$

where dots denote differentiation with respect to time. It is now convenient to introduce the transformations

$$
x_{i}=\sqrt{m_{i}} u_{i}, \quad(i=1, \ldots, n) .
$$

Then the system (3.1) transforms into the system

$$
H: \quad \ddot{x}_{i}=U_{x_{i}}\left(x_{1}, \ldots, x_{n}\right)+f_{i}(t), \quad(i=1, \ldots, n)
$$


where

$$
\begin{aligned}
U\left(x_{1}, \ldots, x_{n}\right) & =O\left(\frac{x_{1}}{\sqrt{m_{1}}}, \ldots, \frac{x_{n}}{\sqrt{m_{n}}}\right), \\
f_{i}(t) & =f_{i}(t) / \sqrt{m_{i}},
\end{aligned}
$$

and the subscript $x_{i}$ denotes partial differentiation with respect to $x_{i}$.

But (3.3) may be regarded as the equations of motion of a mass point having unit mass that moves in the $n$-dimensional space of cartesian coordinates $x_{i},(i=1, \ldots, n)$, called the configuration space. This unit mass point is being accelerated by two forces: One is the conservative force whose components in the $x_{i}$-directions are the $U_{x_{i}}$, and the other is a time-dependent force whose components in the $x_{i}$-directions are the $f_{i}(t)$. The unit mass point and the forces acting on it constitute what may be called the pseudosystem [9].

When moving in the configuration space, the unit mass point traces out a trajectory. Here, we derive the equations of this trajectory. The solutions of (3.3) are of the form

$$
x_{i}=x_{i}(t), \quad(i=1, \ldots, n) .
$$

If one eliminates the parameter $t$ between them, one obtains the equation of the trajectory in the form

$$
x_{j}=x_{j}\left(x_{1}\right), \quad(j=2, \ldots, n)
$$

where $x_{r}=x_{1}$ has been arbitrarily chosen as the independent variable in accordance with (2.18). One finds from (3.5) by direct differentiation

$$
\dot{x}_{j}=x_{j}{ }^{\prime} \dot{x}_{1}, \quad \ddot{x}_{i}=x_{j}{ }^{\prime} \ddot{x}_{1}+x_{j}{ }^{\prime \prime} \dot{x}_{1}{ }^{2}
$$

where primes denote differentiation with respect to $x_{1}$. Moreover, if one regards the velocities as functions of the displacement, the equations of motion $H$ become

$$
\dot{x}_{i} \frac{d \dot{x}_{i}}{d x_{i}}=U_{x_{i}}\left(x_{1}, \ldots, x_{n}\right)+t_{i}(t), \quad(i=1, \ldots, n)
$$

Now, in view of (2.7), every $x_{i}$ takes on its extreme value $X_{i}$ at $t=t_{1}$. Moreover, in view of the continuity and differentiability of strictly* Newtonian

" A system is said to be "strictly Newtonian" if the forces, and hence the accelerations, are bounded for every $t$. Thus, infinite forces of bounded impulse and discontinuities in velocities do not arise in them. 
systems, there always exists some domain $\left[x_{i}, X_{i}\right],(i=1, \ldots, n)$, of the configuration space in which every $x_{i}(t)$ has an inverse

$$
t=t_{i}\left(x_{i}\right) \text {. }
$$

Hence, summing the equations (3.7) over $n$, and integrating them once over the domain $\left[x_{i}, X_{i}\right]$ one finds

$$
\frac{1}{2} \sum_{i=1}^{n}{\dot{x_{i}}}^{2}=U\left(x_{1}, \ldots, x_{n}\right)+h+\sum_{i=1}^{n} F_{i}\left(x_{i}, X_{i}\right)
$$

where $h$ is a constant of integration, and

$$
F_{i}\left(x_{i}, X_{i}\right)=\int_{X_{i}}^{x_{i}} f_{i}\left(t_{i}\left(x_{i}\right)\right) d x_{i} .
$$

It is evident that (3.9) is an equation of the energy balance of the system, and the quantities $F_{i}\left(x_{i}, X_{i}\right),(i=1, \ldots, n)$ represent the work done on the unit mass point by the force components $f_{i}(t)$ in a displacement $\left[x_{i}, X_{i}\right]$. When these forces vanish identically, (3.9) reduces to the energy integral of the autonomous system. The idea of utilizing the inversions (3.8) is also employed in Rauscher's method [3].

The substitution of the first of (3.6) in (3.9) results in an equation in $\dot{x}_{1}{ }^{2}$ which may be explicitly solved for that quantity. That equation together with the system $H$ and the second of (3.6) is sufficient to eliminate the parameter $t$. Hence, the resulting equation is that of the trajectory of the unit mass point. It turns out to be

$$
\begin{aligned}
M: & 2\left[U\left(x_{1}, \ldots, x_{n}\right)+h+\sum_{i=1}^{n} F_{r}\left(x_{r}, X_{r}\right)\right] x_{j}^{\prime \prime} \\
& +\sum_{k=1}^{n} x_{k}{ }^{\prime 2}\left\{x_{i}{ }^{\prime}\left[U_{x_{1}}+f_{1}\left(t\left(x_{1}\right)\right)\right]-\left[U_{x_{j}}+f_{j}\left(t\left(x_{j}\right)\right)\right]\right\}=0, \quad(j=2, \ldots, n)
\end{aligned}
$$

where we have omitted the self-evident subscripts on the $t$. The corresponding equation for the autonomous system is

$M: 2\left[U\left(x_{1}, \ldots, x_{n}\right)+h\right] x_{i}{ }^{\prime \prime}+\sum_{k=1}^{n} x_{k}{ }^{\prime 2}\left[x_{i}{ }^{\prime} U_{x_{i}}-U_{x_{j}}\right]=0, \quad(j=2, \ldots, n)$.

The equation (3.12), but restricted to two degrees of freedom, was probably first given by Kauderer [10]. An equation, equivalent to (3.12) has been given earlier [11]; in it, the arc length $s$ was used as a parameter. 
The elimination of $s$ which has resulted in the compact forms (3.11) and (3.12) was first given by Kinney [12].

The systems of equations (3.11) and (3.12) are denoted as the systems $M$ because they are derivable from a principle akin to the principle of Maupertuis, given below. Similarly, the notation $H$ in (3.3) may recall Hamilton's principle.

\section{The Restricted Principle of Least Action}

It can be readily shown that the system $M$ of the autonomous system; i.e., (3.12) constitutes the Euler-Lagrange equations of the variational principle

$$
\delta A=0
$$

where the action

$$
A=\int_{s_{1}}^{s} \sqrt{U\left(x_{1}, \ldots, x_{n}\right)+h} d s
$$

and

$$
d s^{2}=\sum_{i=1}^{n} d x_{i}{ }^{2}
$$

is the square of the line element in the configuration space. Hence, equations (3.12) follow directly from the principle of Maupertuis.

Now, it is well known that the principle of Maupertuis is only applicable to scleronomous conservative systems. However, if one defines an action

$$
A=\int_{s_{1}}^{s} \sqrt{U\left(x_{1}, \ldots, x_{n}\right)+h+\sum_{i=1}^{n} F_{i}\left(x_{i}, X_{i}\right)} d s
$$

one can show that (3.11) is immediately obtained from the variational principle

$$
\delta A=\mathbf{0}
$$

and from (3.10). Thus one has the [13].

Restricted Principle of Least Action: The natural motion of any system with equations of motion (3.3), on any interval $\left[x_{i}, X_{i}\right],(i=1, \ldots, n)$ in which the inversions (3.8) exist, is such that the action $A$, defined in (3.16), is a minimum. 
While it has only been shown here that the action is stationary, the usual proof that the stationary value is minimum also succeeds here.

The above principle is restricted to the domain $\left[x_{i}, X_{i}\right]$ where the inversions (3.8) exist. In problems of dynamics in general, this domain is not known a priori. However, because of (2.16), (2.17) and (2.18), that domain is, for admissible motions, sufficiently large to contain at least the closed intervals lying between the maximum amplitudes and the equilibrium position, and the corresponding time interval is sufficiently large to cover at least one quarter period. Since the systems $M$ are used directly in the methods to be presented, the restricted principle of least action is of considerable usefulness in problems where admissible motions of admissible systems are examined.

\section{General Description of the Geometrical Method}

The problem in hand has now found a dynamical representation by means of the system $H$ of equations of motion (3.3), and a geometrical one by means of the system $M$ of equations of the trajectories (3.11) and (3.12). It was shown by Darboux [14], for instance, that the correspondence between these for corresponding initial conditions is one-to-one. The essentials of the methods to be used here consist in utilizing the system $M$ to deduce the trajectories and, subsequently, to utilize these trajectories in order to deduce the behavior of the system in the time-displacement domain. This is called "the geometrical method", a term borrowed from Synge's paper [2].

It is interesting to note that the equations $M$ of the trajectory are intrinsically nonlinear, even when the springs are linear. In fact, there exist no admissible systems, autonomous or not, for which the equations of the trajectory become linear. The assumption of linear springs implies merely that all $U_{x_{i}}$ are of the form

$$
U_{x_{i}}=\sum_{i=1}^{n} a_{i j} x_{j}, \quad(i=1, \ldots, n)
$$

where the $a_{i j}$ are either constants or functions of $t$. Under it, equations (3.3) become linear while (3.11) and (3.12) remain nonlinear. The intrinsic nonlinearity of the system $M$ will perhaps be better appreciated if on recalls that its integrals are Lissajous curves. These may have many points of self-crossing and may even be compact on some domain of the configuration space. One may conclude that admissible systems with linear springs are more easily examined by means of the system $H$ of equations of motion than by $M$. The fortunate circumstance that the superposition theorem holds in this case can, then, always be utilized to find the general motions and, if one wants to work very hard, their trajectories in the configuration space [15]. 
While linearity of the physical system has no essential simplifying effects on the system $M$, nonlinearity has no fearful, complicating effects on $M$, either. Hence, if these equations can be used to deduce solutions of the physically linear problem, they can frequently be used with equal ease to deduce those of physically nonlinear problems [16].

Now, it is quite possible that a system of very difficult, nonlinear differential equations have certain simple integrals. In fact, we shall demonstrate that the trajectories of admissible motions are such simple integrals. They are of the form

$$
x_{i}=x_{i p}\left(x_{1}\right), \quad(i=2, \ldots, n), \quad(p=1,2, \ldots)
$$

where the index $p$ denotes the mode of vibration. Say, the trajectory corresponding to the $m$ th mode of admissible motion is given by the functions

$$
x_{i}=x_{i m}\left(x_{1}\right), \quad(i=2, \ldots, n)
$$

that satisfy the system $M$, and which are supposedly known. Then, their substitution in the first equation of the system $H$, for instance, results in

$$
\ddot{x}_{1}=U_{x_{1}}\left(x_{1}, x_{2 m}\left(x_{1}\right), \ldots x_{n m}\left(x_{1}\right)\right)+f_{1}(t),
$$

an equation in $x_{1}$ only.

If the system is autonomous, $f_{1}(t) \equiv 0$, and (4.4) is of the form

$$
\ddot{x}_{1}=g\left(x_{1}\right) \text {. }
$$

Then, for initial conditions (for instance)

$$
x_{1}(0)=X_{1}, \quad \dot{x}_{1}(0)=0,
$$

and with the definition

$$
\int_{X_{1}}^{x_{1}} g(u) d u=G\left(x_{1}, X_{1}\right)
$$

the integral of $(4.4)$ becomes

$$
t\left(x_{1}\right)=\int_{X_{1}}^{x_{2}}\left\{2\left[G\left(v, X_{1}\right)-G\left(X_{1}, X_{1}\right)\right]\right\}^{-1 / 2} d v,
$$

and the period of the motion is

$$
T_{m}=4 \int_{X_{1}}^{0}\left\{2\left[G\left(v, X_{1}\right)-G\left(X_{1}, X_{1}\right)\right]\right\}^{-1 / 2} d v .
$$


If the system is nonautonomous, and the amplitude of the forcing function is small; i.e.,

$$
f_{1}(t)=\varepsilon f_{1}(t), \quad|\varepsilon| \text { small, }
$$

the steady-state forced vibration problem can be shown to become a perturbation problem on that of free vibrations in admissible motion. It is evident, then, that the central task in either problem (autonomous, or forced) consists in determining the trajectories of admissible motions of autonomous, admissible systems.

\section{Trajectories of Admissible Motion}

\section{General Properties}

We shall assume that, after formal reduction to a first-order system, the right-hand side of (3.3) satisfy a Lipschitz condition. Trajectories then exist and all are continuous. Because of $(2.16)$, every admissible trajectory passes through the origin of the configuration space. In view of (2.17), the equation (3.9) reduces at $t=t_{1}$ to

$$
U\left(x_{1}, \ldots, x_{n}\right)+h+\sum_{i=1}^{n} F_{i}\left(x_{i}, X_{i}\right)=0 .
$$

This equation defines a surface in configuration space called the $L$-surface, or the bounding surface. Then, it follows from (2.16) that every admissible trajectory intercepts the L-surface. The equation (2.18) states that the $x_{i}\left(x_{r}\right)$ are single-valued for every $i$ and any $r$. Hence, for instance, $x_{k}\left(x_{l}\right)$ is singlevalued, and the same is true for its inverse $x_{l}\left(x_{k}\right)$. But this requires that each be monotonic. We denote this property by saying that every admissible trajectory is strictly monotonic. Finally, since trajectories satisfy the EulerLagrange equations of (3.17), existence of real trajectories requires that the quantity under the square root of $(3.16)$ be non-negative. But, it is seen from (5.1), that this quantity vanishes on the $L$-surface. Then, it follows from the properties of $U$ and the $F_{i}$ that no admissible trajectory can pierce the L-surface. These properties are summarized in

Theorem V-I: Every continuous, strictly monotonic curve, defined by solutions of (3.11) or (3.12), which passes through the origin of the configuration space and which terminates on the L-surface (5.1) is that of a vibration-inunison as defined in (2.15) to (2.18).

From the preceding remarks it follows that the unit mass point of the pseudo-system oscillates back and forth along this trajectory, and the period of this oscillation is the same as that of the vibration-in-unison of the physical system. 
If the vibrations-in-unison are similar as defined in (2.19), the corresponding trajectories are defined by

$$
x_{i}\left(x_{1}\right)=c_{i 1} x_{1}, \quad(i=2, \ldots, n)
$$

where the $c_{i 1}$ are constants. In that case, (3.11) reduces to

$$
x_{j}^{\prime}\left[U_{x_{1}}+f_{1}\left(t\left(x_{1}\right)\right)\right]=U_{x_{j}}+f_{j}\left(t\left(x_{j}\right)\right), \quad(j=2, \ldots, n)
$$

because

$$
\sum_{k=1}^{n} x_{k}{ }^{2}>0
$$

But, (5.3) may be recast in the form

$$
\frac{d x_{1}}{U_{x_{1}}+f_{1}\left(t\left(x_{1}\right)\right)}=\ldots=\frac{d x_{n}}{U_{x_{n}}+f_{n}\left(t\left(x_{n}\right)\right)} .
$$

It follows that the differential equations of similar vibrations-in-unison satisfy the system (5.3) or (5.4).

All properties of admissible trajectories, given above, hold whether the system is autonomous or not. Additional properties of admissible trajectories can be deduced when the system is autonomous.

\section{Admissible Trajectories of Autonomous Systems}

The equations of autonomous systems are obtained from those above by putting

$$
f_{i}(t)=F_{i}\left(x_{i}, X_{i}\right) \equiv 0, \quad(i=1, \ldots, n) .
$$

In that case, the energy integral

$$
T\left(\dot{x}_{1}, \ldots, \dot{x}_{n}\right)-U\left(x_{1}, \ldots, x_{n}\right)=h
$$

is seen from (3.9) to exist where

$$
T\left(\dot{x}_{1}, \ldots, \dot{x}_{n}\right)=\frac{1}{2} \sum_{i=1}^{n} \dot{x}_{i}^{2}
$$

is the kinetic energy of the pseudo-system, and $h \geqslant 0$ is the energy level of a given motion. The system $M$ of the autonomous case is given in (3.12), and the corresponding action integral is (3.14).

For this case, the bounding surface is defined by

$$
U\left(x_{1}, \ldots, x_{n}\right)+h=0,
$$


and this is also the maximum equipotential surface because (5.8) coincides with (5.6) when the kinetic energy vanishes. In order to call attention to the fact that (5.8) defines the bounding surface when the system is autonomous, it will be denoted as the $L_{a}$-surface.

From (2.5), (2.6), (2.7), and (5.8) it follows that:

(i) the $L_{a}$-surface is a closed, smooth surface surrounding the origin of the configuration space, and star-shaped with respect to it [9].

From (2.8) one sees that

(ii) the $L_{a}$-surface is symmetric with respect to the origin of the configuration space.

From the admissibility condition (2.6) and (5.8) it is evident that

(iii) the quantity $U+h>0$ at the origin of the configuration space. This quantity does not change sign in the open, finite domain $D_{a}$ bounded by the $L_{a}$-surface, it vanishes on $L_{a}$, and it is negative (at least near the $L_{a}$-surface) in the infinite open domain bounded by the $L_{a}$-surface.

These properties lead immediately to

Theorem V-II: Every trajectory, admissible or not, defined by solutions $x_{i}\left(x_{1}\right),(i=2, \ldots, n)$ of (3.12), lies in the closed, finite domain $D_{a}$ of the configuration space, bounded by the $L_{a}$-surface defined in (5.8).

It is not necessary that all trajectories actually attain the $L_{a}$-surface. However, if they do, this occurs necessarily at an instant $t_{1}$ when all $\dot{x}_{i}\left(t_{1}\right)$ vanish. Trajectories which attain the $L_{a}$-surface will be called trajectories of motion with a rest point [19]. Not all motions with a rest point need be admissible because the corresponding trajectories need not pass through the origin. However, all admissible motions are motions with a rest point.

Next we state a property, first observed by Mawhin [6]. It is

Theorem V-III: If $\mathscr{E}$ is any trajectory, admissible or not, of an admissible, autonomous system, the trajectory $\mathscr{E}^{\prime}$, symmetric to $\mathscr{E}$ with respect to the origin, is also a trajectory.

The proof follows immediately from (2.8) and its consequence

$$
U_{x_{i}}\left(-x_{1}, \ldots,-x_{n}\right)=-U_{x_{i}}\left(x_{1}, \ldots, x_{n}\right) \text {. }
$$

Of importance to admissible trajectories is $[6]$ the

Corollary V-IV: Every trajectory, admissible or not, (of an admissible, autonomous system) which passes through the origin is symmetric with respect to it. 
Finally, we have

Theorem V-V: If a trajectory, admissible or not, of an admissible, autonomous system attains the $L_{a}$-surface, it intercepts that surface orthogonally. To prove it we observe that the system $M$ of autonomous systems, given in (3.12), reduces on the $L_{a}$-surface to

$$
x_{j}{ }^{\prime} U_{x_{1}}=U_{x_{j}}, \quad(j=2, \ldots, n),
$$

and this may be cast in the form

$$
\frac{d x_{1}}{U_{x_{1}}}=\ldots=\frac{d x_{n}}{U_{x_{n}}} .
$$

But equations (5.11) are precisely the conditions for orthogonal intersection.

Special additional properties hold for similar vibrations-in-unison of autonomous systems. They are given in [9].

Theorem V-VI: Every trajectory of a similar vibration-in-unison of an admissible autonomous system intersects every equipotential surface orthogonally, and [9]

Theorem V-VII: If there exists a straight line which intersects every equipotential surface of an admissible, autonomous system orthogonally, it is a trajectory.

To prove these theorems, we observe that equipotential surfaces are defined by

$$
U\left(x_{1}, \ldots, x_{n}\right)+h^{*}=0, \quad\left(0<h^{*} \leqslant h\right)
$$

and, in view of the properties of admissible potential functions, the equipotential surfaces are simple, closed, non-intersecting surfaces surrounding the origin, and compact on $D_{a}$.

It follows from (5.2) that, in the case of similar motion,

$$
x_{i}^{\prime \prime}\left(x_{1}\right) \equiv 0, \quad(i=2, \ldots, n) .
$$

Hence, the system (3.12) reduces throughout $D_{a}$ to $(5.10)$ or (5.11). Now, the total differential of every equipotential surface $d\left(U+h^{*}\right)$ is independent of $h^{*}$ which proves the first of the theorems. It is clear that the second theorem also holds because (5.11) is merely one way of writing the system $M$ when equations (5.13) are satisfied. 


\section{A Nere Definition of Normal Modes}

It was observed in connection with the definition of admissible motions that the normal mode vibrations of linear, autonomous systems are in fact vibrations-in-unison. They are usually deduced from the dynamical system by means of eigenvalue theory, and in the proof of their existence the fact is utilized that the potential function $U$ is a quadratic form [17]. Now, it is the very essence of nonlinearity (as defined here) that the potential function is not a quadratic form; hence, the application of conventional methods for deducing normal mode vibrations is difficult to generalize to nonlinear systems.

We shall show that the normal mode vibrations of linear systems may be defined in terms of admissible trajectories, and that these are deducible from the system $M$, rather than $H$. In fact, the normal mode vibrations of autonomous systems are its only vibrations-in-unison.

Furthermore, it will be demonstrated that nonlinear admissible systems also possess such vibrations-in-unison. The existence proof is given in the appendix. These observations permit a new definition of normal mode vibrations which is wide enough to include the well-known normal-mode vibrations of linear systems as a special case.

Definition I: Vibrations-in-unison of an admissible, autonomous system are the normal-mode vibrations of that system.

We shall also use

Definition II: Trajectories in the configuration space corresponding to normal-mode vibrations of admissible, autonomous systems are called modal lines.

Then, one sees from Theorem V-I that modal lines are strictly monotonic curves passing through the origin, and terminating on the $L_{a}$-surface.

\section{Straight Modal Lines}

In (2.20), it was noted that the vibrations-in-unison of the linear, autonomous system are similar or, that the modal lines of that system are straight. Since we wish to deduce these vibrations by the geometrical method, we derive first the conditions, necessary and sufficient for the existence of straight modal lines, and we demonstrate subsequently that the linear, autonomous, admissible system satisfies these conditions. The derivation of the n.a.s. conditions is conveniently done by transforming 
from cartesian to spherical coordinates in $n$-space. These transformations are of the form [11]

$$
x_{i}=r f_{i}\left(\theta_{1}, \ldots, \theta_{n-1}\right), \quad(i=1, \ldots, n)
$$

and, specifically, they are [9]

$$
x_{i}=r \sin \theta_{n+1-i} \prod_{i=1}^{n-i} \cos \theta_{j}, \quad \theta_{n}=\pi / 2, \quad(i=1, \ldots, n) .
$$

When the equation of the $L_{a}$-surface is transformed into spherical coordinates, it is of the form

$$
\hat{O}\left(r, \theta_{1}, \ldots, \theta_{n-1}\right)+h=0
$$

Then, one has [9] the

Theorem VI-I: The conditions, necessary and sufficient for the existence of at least one mode of similar normal-mode vibration of an admissible, autonomous system are that every partial derivative of $\hat{\theta}$ with respect to the $\theta_{i}$ be of the form

$$
\hat{0}_{\theta_{i}}=\Theta_{i 1}\left(r, \theta_{1}, \ldots, \theta_{n-1}\right) \cdot \Theta_{i 2}\left(\theta_{1}, \ldots, \theta_{n-1}\right), \quad(i=1, \ldots, n-1),
$$

and that the equations

$$
\Theta_{i 2}\left(\theta_{1}, \ldots, \theta_{n-1}\right)=0, \quad(i=1, \ldots, n-1)
$$

have at least one system of real roots $\theta_{i}^{*}$.

To sketch the proof, we observe that, in spherical coordinates, the equipotential surfaces are given by

$$
\hat{U}\left(r, \theta_{1}, \ldots, \theta_{n-1}\right)+h^{*}=0, \quad 0<h^{*} \leqslant h .
$$

Now, radius vectors $r$ to points on equipotential surfaces having stationary distances from the origin with respect to neighboring points on these same surfaces satisfy

$$
d r=0,
$$

and these radius vectors intersect the equipotential surfaces orthogonally. From (6.3)

$$
\hat{v}_{r} d r+\sum_{i=1}^{n-1} \hat{\theta}_{\theta_{i}} d \theta_{i}=0
$$

or in view of (6.7)

$$
\hat{0}_{\theta_{i}}=0, \quad(i=1, \ldots, n-1) .
$$


The locus of these points of stationary distance on the equipotential surfaces is a straight line if, and only if, the system (6.9) has roots independent of $r$ which requires that the $\hat{\sigma}_{\theta_{i}}$ be of the form (6.4).

Theorem VI-I is useful not only because it may be used readily to test whether straight modal linescan exist, but the direction-cosines of the straight modal lines, if they exist, are furnished by the roots of (6.5). Hence, the theorem provides also a method for computing the straight modal lines.

It can be shown that the new Definition I of normal mode vibrations yields only the normal modes, and all of the normal modes of admissible, autonomous, linear systems [6].

\section{Interpretation of Modal Lines [11]}

The modal lines have a very simple, geometrical significance which will be illustrated here by means of an admissible, autonomous system having the three degrees of freedom

$$
x_{1}=x, \quad x_{2}=y, \quad x_{3}=z .
$$

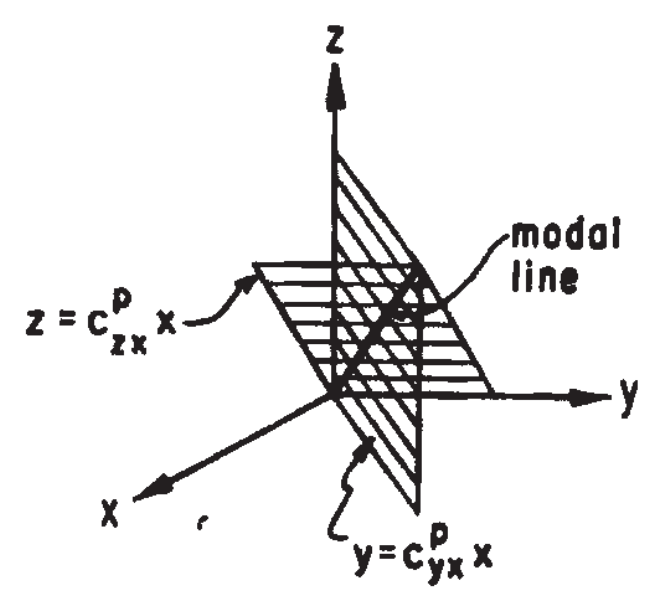

(a)

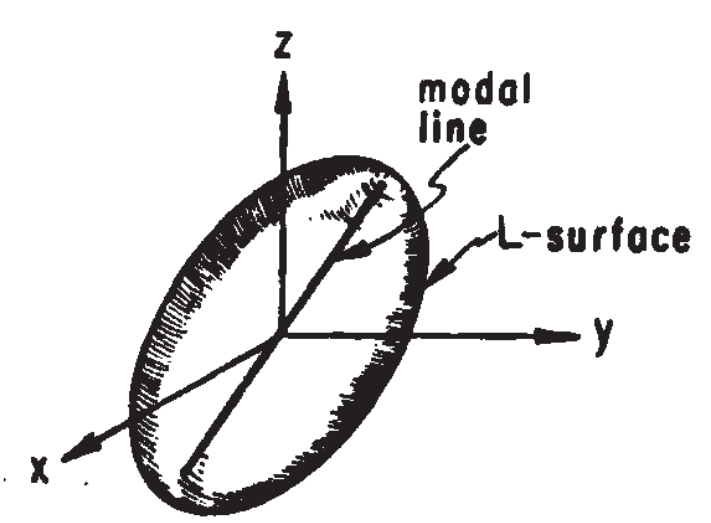

(b)

FIG. 2. Modal line of linear system with three degrees of freedom.

Suppose the system is linear. Then, the equipotential surfaces are given by the concentric three-dimensional ellipsoids (with center at the origin)

$$
\frac{1}{2}\left(x^{2}+y^{2}+z^{2}\right)=h^{*}, \quad 0<h^{*} \leqslant h,
$$

and the vibration in the pth normal mode is given by

$$
x=X_{p} \cos \omega_{p} t, \quad y=Y_{p} \cos \omega_{p} t, \quad z=Z_{p} \cos \omega_{p} t
$$

where $X_{p}, Y_{p}, Z_{p}$ are constants.

It follows from these equations that the $p$ th normal mode satisfies

$$
y / x \equiv Y_{p} \mid X_{p}=c_{y x,}^{p}, \quad z / x \equiv Z_{p} / X_{p}=c_{z s}^{p} .
$$


Then, the modal line in the $p$ th mode is the intersection of the planes

$$
y=c_{y x}^{p} x, \quad z=c_{x x}^{p} x .
$$

Evidently, this intersection is a straight line passing through the origin. From the known geometric properties of ellipsoids and from Theorem V-VII it follows that there exist three such modal lines, and they are the principal

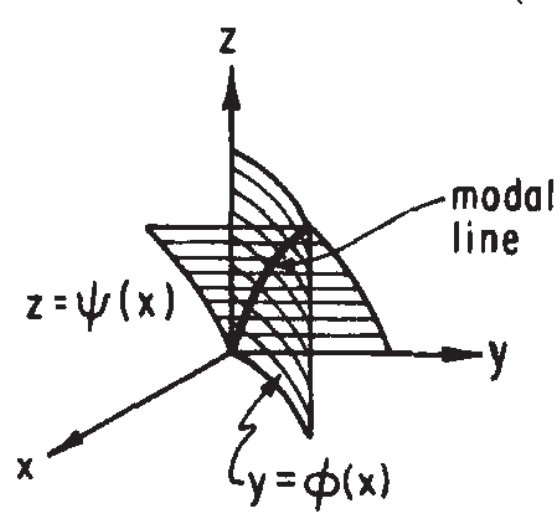

(a)

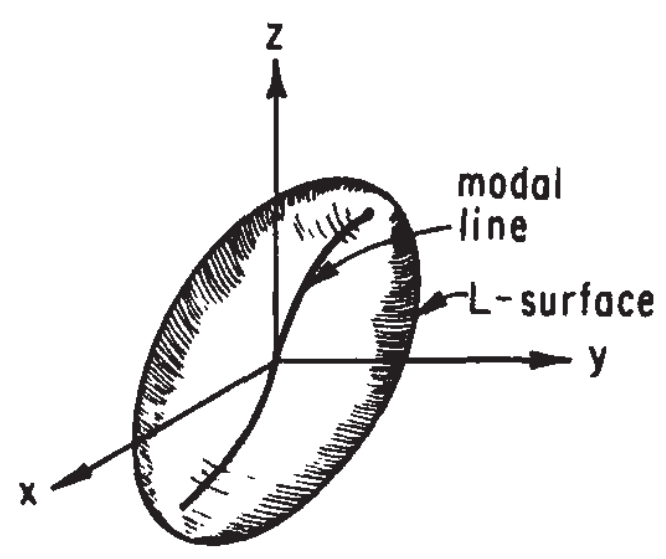

(b)

Fig. 3. Modal line of nonlinear system with three degrees of freedom.

axes of the ellipsoid. The construction of a modal line, and its location inside the bounding ellipsoid is shown in Figures 2(a) and (b).

If the problem is nonlinear, but the normal mode vibrations are similar, equations (6.14) still apply, but, in that case, the bounding ovaloid is no longer an ellipsoid.

If the problem is nonlinear, and the normal-mode vibrations are nonsimilar, equations (6.14) are replaced by equations of the form

$$
y=\varphi(x), \quad z=\psi(x)
$$

where $\varphi$ and $\psi$ are nonlinear, monotonic functions of $x$. Each is a cylinder in $x y z$-space containing the origin, as shown in Figure 3(a). Their intersection is a modal line, and this modal line inside the bounding ovaloid is shown in Figure 3(b).

\section{Properties of Motions with a Rest Point $[19,24]$}

\section{The Transversals (or P-curves)}

Motions with a rest point have been defined as those in which there exists a time $t_{1}$ at which the velocity of all mass points of the physical system vanishes. If the system is autonomous, the trajectories corresponding to motions with a rest point are those which attain the $L_{a}$-surface, and ad- 
missible motions belong to this class. The equipotential surfaces of these systems are given by (5.12), and the transversals are defined in terms of these equipotential surfaces. Transversals, or $P$-curves, are the lines orthogonal to the equipotential surfaces. From their definition, one has immediately

Theorem VII-I: At a point $\left(x_{1}, \ldots, x_{n}\right)$ of the configuration space where the slope of a transversal is well-defined, it coincides with the direction of the force which acts, at that point, on the unit mass point of any admissible, autonomous pseudo-system.

Hence, the $P$-curves have an important dynamical significance, and it is of interest to determine their properties. One observes that, when $n>1$, the transversals constitute a family of curves which pass through the origin of the $n$-space, which are compact on $D_{a}$, and which are nonintersecting, except at the origin. It would be interesting to determine their geometrical properties in the entire domain $D_{a}$. However, this is a difficult task when the number of degrees of freedom exceeds two; therefore, our attention will be restricted to the case $n=2$.

Consider an admissible, autonomous system having the degrees of freedom

$$
u_{2}=u, \quad u_{2}=v
$$

We admit linearizable and certain nonlinearizable systems by writing

$$
\frac{\partial}{\partial u} \mathcal{O}(u, v)=-\bar{a} u^{k}-\bar{b} v^{k}+\bar{P}(u, v),
$$

$$
\frac{\partial}{\partial v} \sigma(u, v)=-\bar{c} u^{k}-d v^{k}+Q(u, v)
$$

where $k$ is a positive, odd integer, and $P$ and $Q$ are of degree higher than $k$. The determinant

$$
\left|\begin{array}{ll}
\bar{a} & \bar{b} \\
\bar{c} & d
\end{array}\right| \neq \mathbf{0} .
$$

With these definitions, the system is linearizable if $k=1$, and nonlinearizable if $k>1$.

The equations of motion of the physical system are

$$
m_{1} \ddot{i}=\frac{\partial}{\partial u} \delta(u, v),
$$

$$
m_{2} \ddot{v}=\frac{\partial}{\partial v} \sigma(u, v)
$$


those of the pseudo-system are

$$
\begin{aligned}
& \ddot{x}=U_{x}(x, y), \\
& \ddot{y}=U_{y}(x, y),
\end{aligned}
$$

and the equation of the trajectories is

$$
2[U(x, y)+h] y^{\prime \prime}+\left(1+y^{\prime 2}\right)\left[y^{\prime} U_{x}(x, y)-U_{y}(x, y)\right]=0
$$

where primes denote differentiation with respect to $x$.

The system possesses the energy integral

$$
T(\dot{x}, \dot{y})-U(x, y)=h .
$$

The bounding surface (here a curve) is the locus of the points $P(X, Y)$ for which the kinetic energy vanishes when the energy level of the motion is $h$, and the equation of the $L_{a}$-curve is

$$
U(X, Y)+h=0 .
$$

The equipotential curves are the functions

$$
y=\varphi(x)
$$

which satisfy the equation

$$
U(x, \varphi)+h^{*}=0, \quad 0<h^{*} \leqslant h
$$

and they will be called $E$-curves. By differentiating (7.10), one finds that $E$-curves satisfy the differential equation

$$
\frac{d \varphi}{d x}=-\frac{U_{x}(x, \varphi)}{U_{\varphi}(x, \varphi)}
$$

It follows that the transversals, or $P$-curves, are the functions

$$
y=\theta(x)
$$

which satisfy the differential equation

$$
\frac{d \theta}{d x}=\frac{U_{\theta}(x, \theta)}{U_{x}(x, \theta)}
$$

We observe that (7.13) is singular only at the origin, and the equation of the trajectories (7.6) is singular only on the $L_{a}$-curve. Consequently, we define as regular points of the $x y$-plane all points, except the origin, which lie in the open, finite domain $D_{a}$ bounded by the $L_{a}$-curve. 
To begin with, we prove

Theorem VII-II: The origin is always a node for the P-curves of admissible, autonomous systems having two degrees of freedom.

Suppose the system is linearizable [19], or $k=1$ in (7.2). Then, in the neighborhood of the origin,

$$
U_{x}=x U_{x x}(0,0)+y U_{x y}(0,0)+\ldots,
$$

$$
U_{y}=x U_{x y}(0,0)+y U_{y y}(0,0)+\ldots
$$

The substitution of these in the equation (7.11) of the $E$-curves results in

$$
\frac{d \varphi}{d x}=\frac{-x U_{x x}-\varphi U_{x \varphi}+\ldots}{x U_{x \varphi}+\varphi U_{\varphi \varphi}+\ldots}
$$

where the partial derivatives are evaluated at the origin. But, the integrals of (7.11), and hence (7.15), are known; they are $E$-curves and they form a continuum of simple, closed, nonintersecting curves surrounding the origin. Then, it follows that the origin is a center for the $E$-curves and this requires, by the Poincaré theory of singular points [1], that

$$
U_{x x} U_{\varphi \varphi}-U_{x \varphi}^{2}>0 .
$$

The substitution of (7.14) in the equation (7.13) of the $P$-curves results in

$$
\frac{d \theta}{d x}=\frac{x U_{x \theta}+\theta U_{\theta \theta}+\ldots}{x U_{x x}+\theta U_{x \theta}+\ldots} .
$$

Application of the Poincaré theory to this equation has as immediate consequences

$$
\begin{gathered}
U_{x x}+U_{\theta \theta} \neq 0, \\
\left(U_{x x}-U_{\theta \theta}\right)^{2}+4 U_{x \theta}^{2}>0 .
\end{gathered}
$$

These are two of the three conditions which are necessary for the origin to be a node. But, in view of (7.16),

$$
U_{x x} U_{\theta \theta}-U_{x \theta}^{2}>0 .
$$

This is the third necessary condition, and (7.18) and (7.19) are also sufficient. Hence, Theorem VII-II is satisfied in the linearizable case. 
To prove the theorem for nonlinearizable systems [21], we note that, in the neighborhood of the origin, the potential function of the pseudosystem is

$$
U(x, y)=-\frac{a}{k+1}\left(\frac{x}{\sqrt{m_{1}}}\right)^{k+1}-\frac{c}{k+1}\left(\frac{y}{\sqrt{m_{2}}}\right)^{k+1}
$$

$$
-\frac{b}{k+1}\left(\frac{x}{\sqrt{m_{1}}}-\frac{y}{\sqrt{m_{2}}}\right)^{k+1}+\ldots
$$

where $a, b, c>0$ are constants.

The derivatives of this function (with $y$ replaced by $\theta$ ) are now substituted in the equation of the $P$-curves (7.13), and the transformations

$$
x / \sqrt{m_{1}}=\xi, \quad y / \sqrt{m_{2}}=\eta
$$

are introduced in the resulting equation. Then, one finds, instead of (7.17) in the neighborhood of the origin

$$
\frac{d \eta}{d \xi}=\frac{-c \eta^{k}+b(\xi-\eta)^{k}+\ldots}{-a \xi^{k}-b(\xi-\eta)^{k}+\ldots} .
$$

The singularity at the origin of this equation may be discussed by the method of Argemi and Sideriadès $[\mathbf{2 2}, \mathbf{2 3}]$. These authors consider the equation

$$
\frac{d y}{d x}=\frac{Y(x, y)}{X(x, y)}
$$

where $X$ and $Y$ are homogeneous functions in $x$ and $y$ of the same degree $k$. They introduce a transformation

$$
y=x \lambda(x)
$$

under which the functions go over into

$$
X=x^{k} f(\lambda), \quad Y=x^{k} g(\lambda)
$$

and the differential equation (7.23) becomes

$$
\frac{d \lambda}{d x}=\frac{h(\lambda)}{x f(\lambda)}
$$

where $h(\lambda)=g(\lambda)-\lambda f(\lambda)$. Now, this last equation has simple singular points on the $\lambda$-axis at the zeros $\lambda_{i}$ of $h(\lambda)$, and their discussion is accessible to the Poincare theory. Expansion of the numerator and denominator of the right-hand side of (7.26) near the singular points gives 


$$
\frac{d \lambda}{d x}=\frac{h^{\prime}\left(\lambda_{i}\right) \lambda+\ldots}{f\left(\lambda_{i}\right) x+\ldots}
$$

The authors show that only saddles and nodes can occur, and one has

$$
\begin{array}{ll}
\text { a node if } & f\left(\lambda_{i}\right) h^{\prime}\left(\lambda_{i}\right)>0, \\
\text { a saddle if } & f\left(\lambda_{i}\right) h^{\prime}\left(\lambda_{i}\right)<0 .
\end{array}
$$

The quantity $f\left(\lambda_{i}\right) h^{\prime}\left(\lambda_{i}\right)$ does not vanish, in general, because the zeros of $f(\lambda)$ do not coincide with those of $h(\lambda)$ when $X \neq Y$, and $h^{\prime}(\lambda)$ does not vanish at the zeros of $h(\lambda)$. Moreover, when the $\lambda_{i}$ are ordered, according

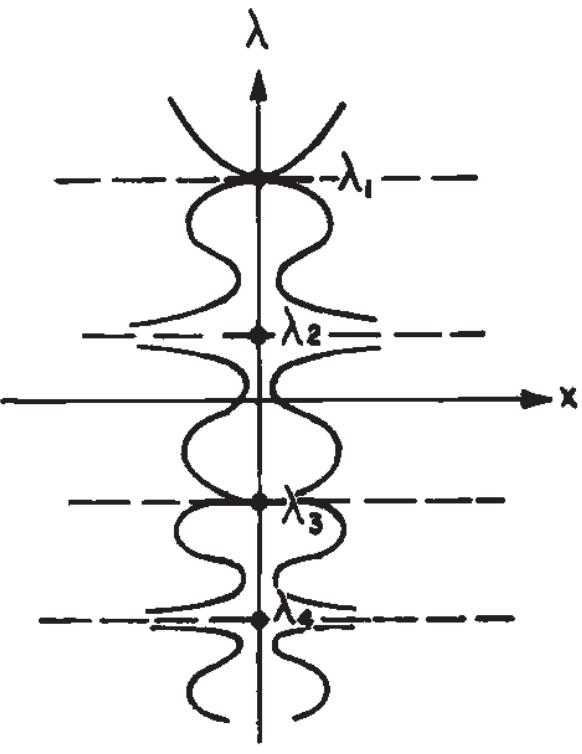

(a)

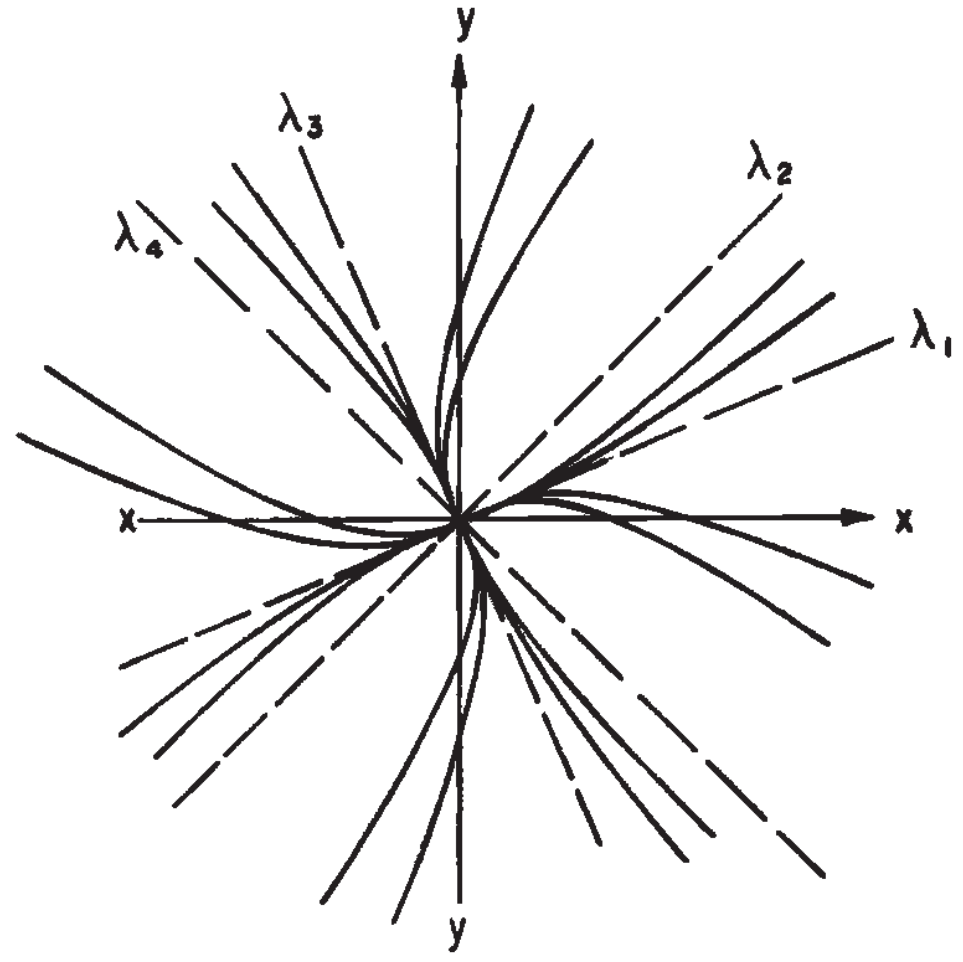

(b)

FIG. 4. Saddles and nodes in $x \lambda$-plane and mapping on configuration space.

to their magnitudes (in ascending or descending order), nodes and saddles occur alternately. Clearly, the inverse of the transformation (7.24) maps all singular points of (7.27) into the origin of the $x y$-plane, and the zeros $\lambda_{i}$ of $h(\lambda)$ are the slopes of the trajectories at the origin. This proves Theorem VII-II for the case of nonlinearizable systems.

It may be interesting to follow the construction in greater detail, as done in Figure 4. Let $\lambda_{1}, \lambda_{3}, \ldots$ give the nodes in the $x \lambda$-plane, and $\lambda_{2}, \lambda_{4}, \ldots$ the saddles. Next, construct the straight lines that pass through the origin with slopes $\lambda_{i},(i=1,2, \ldots)$ and assume that $\lambda_{1}>0$; i.e., the line having this slope lies in the first and third quadrants. Next consider the trajectories lying in the sector enclosed by the lines of slopes $\lambda_{1}$ and $\lambda_{2}$ (starting in the 
first quadrant and proceeding in a counterclockwise direction). This sector contains a continuum of trajectories that pass with slope $\lambda_{1}$ through the origin, and they tend to lines parallel to the line of slope $\lambda_{2}$. The latter is a degenerate trajectory of this class. The trajectories in the next sector between the lines of slopes $\lambda_{2}$ and $\lambda_{3}$ pass through the origin with slope $\lambda_{3}$ and tend to lines parallel to the line of slope $\lambda_{2}$, that line being also a degenerate trajectory of this class. This process is continued until the $x y$-plane is filled in the neighborhood of the origin with trajectories. In Figure 4(a), the trajectories and singular points of the $x \lambda$-plane are shown, and in Figure $4(\mathrm{~b})$, the corresponding picture in the $x y$-plane is shown.

Suppose, as a special example, that $k=3$ in (7.20) and (7.22). Then, [24] a simple computation shows that

$$
\begin{aligned}
& f(\lambda)=-b\left[(2-\beta)+(1-\lambda)^{3}\right], \\
& h(\lambda)=-b\left[\lambda^{4}-\alpha \lambda^{3}+\beta \lambda-1\right]
\end{aligned}
$$

where

(7.30)

$$
\alpha=2-\frac{c}{b},
$$

$$
\beta=2-\frac{a}{b} .
$$

It is now necessary to determine first the zeros $\lambda_{i}$ of $h(\lambda)$ and, next, the sign of the product $f h^{\prime}$ at these zeros. Writing $h(\lambda)=0$ in the form

$$
\lambda^{4}-1=\left(\alpha \lambda^{2}-\beta\right) \lambda
$$

one sees that the left-hand side is even in $\lambda$ and has two real zeros at $\lambda= \pm 1$, and the right-hand side is odd and has one or three real zeros. Hence, these two functions have always at least two real intersections, or $h(\lambda)$ has always at least two real zeros. One may assume without loss of generality* that $c \geqslant a$. Now, it follows from the second of (7.29) and from the assumptions on $a, b$, and $c$, that

$$
\begin{aligned}
h(\infty) & =h(-\infty)=-\infty, \\
h(0) & =b, \\
h(1) & =-b(-\alpha+\beta)=-(c-a) \leqslant 0, \\
h(-1) & =-h(1)=c-a \geqslant 0 .
\end{aligned}
$$

* If, on the contrary $c<a$, we simply denote the displacement of $m_{1}$ by $v$ and that of $m_{\mathrm{a}}$ by $u$ which restores the assumed relation. 
Hence, $h(\lambda)$ looks as shown in Figure 5 (where no attention is to be paid to the location of the maximum). It follows from (7.32) and Figure 5 that

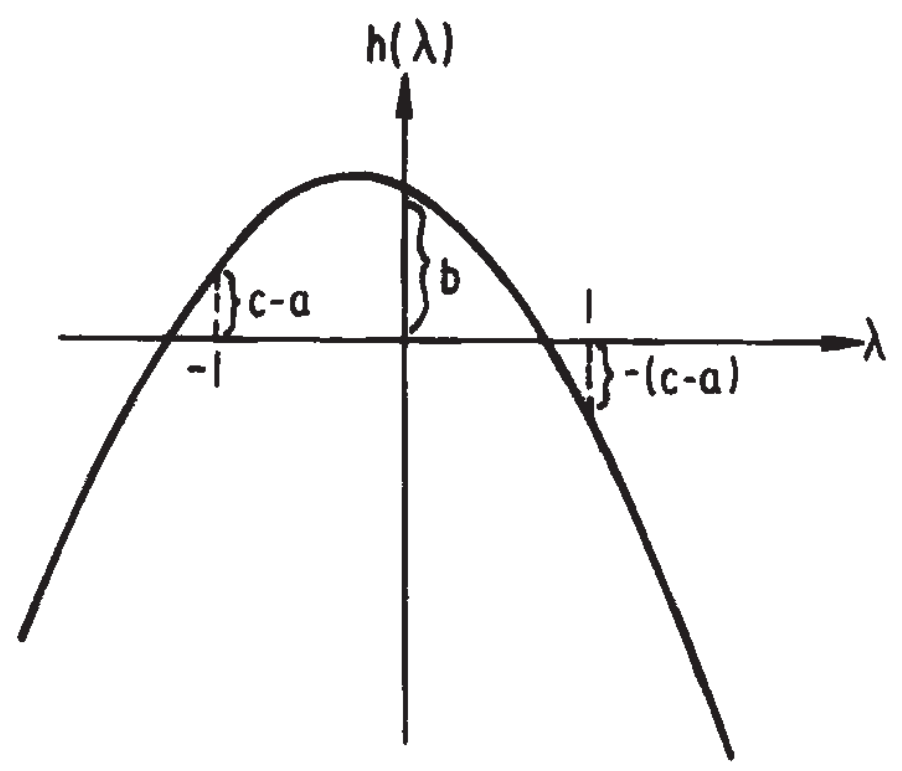

FIg. 5. The curve $h(\lambda)$.

one root, say $\lambda_{1}$, is positive and lies in the interval $0<\lambda_{1}<1$, and $\lambda_{2}$ is negative. Moreover, the slopes are

$$
\begin{aligned}
& h^{\prime}\left(\lambda_{1}\right)<0, \\
& h^{\prime}\left(\lambda_{2}\right)>0 .
\end{aligned}
$$

Next, we examine $f(\lambda)$. It follows from the first of (7.29) that

$$
\begin{gathered}
f(\infty)=\infty, \\
f(-\infty)=-\infty,
\end{gathered}
$$

and that $f(\lambda)$ has only a single real zero at $\lambda=1-\sqrt[3]{2-\beta}$. To evaluate the $f\left(\lambda_{i}\right)$, it is convenient to return to the physical constants, so that

$$
f(\lambda)=-b\left[\frac{a}{b}+(1-\lambda)^{\mathrm{s}}\right] .
$$

Since $b>0, a / b>0$, and $0<\lambda_{1}<1$, and since $\lambda_{2}<0$, one has from (7.35)

$$
\begin{aligned}
& f\left(\lambda_{1}\right)<0, \\
& f\left(\lambda_{2}\right)<0 .
\end{aligned}
$$

Combining (7.33) and (7.36),

$$
\begin{aligned}
& f\left(\lambda_{1}\right) h^{\prime}\left(\lambda_{1}\right)>0, \\
& f\left(\lambda_{2}\right) h^{\prime}\left(\lambda_{2}\right)<0 .
\end{aligned}
$$


Hence, the $x \lambda$-plane has, at least, two singular points on the $\lambda$-axis. One lies on the positive branch between 0 and 1 , and it is a node. The other lies on the negative branch, and it is a saddle.

We shall assume that $\lambda_{1,2}$ are the only zeros* of $h(\lambda)$. Under this assumption we have now demonstrated

Theorem VII-II: When $k=1,3$ and $h(\lambda)$ has only two zeros, all transversals of admissible, autonomous systems pass through the origin of the $x y$ plane with common slope $\theta_{0}^{\prime}$.

To determine the properties of $P$-curves not near the origin, we determine the locus of their inflection points, if any. By differentiating (7.13), one has

$$
\theta^{\prime \prime}=\frac{U_{x \theta}\left(U_{x}^{2}-U_{\theta}^{2}\right)-U_{x} U_{\theta}\left(U_{x x}-U_{\theta \theta}\right)}{U_{x}^{3}} .
$$

This locus will be called the F-curve:

$$
F(x, y) \equiv U_{x y}\left(U_{x}^{2}-U_{y}^{2}\right)-U_{x} U_{y}\left(U_{x x}-U_{y y}\right)=0 .
$$

It follows from (7.39) that $F$-curves pass through, and are symmetric with respect to, the origin.

Next we determine the locus of points such that the tangents to $P$ curves at these points pass through the origin. Evidently, these are the points for which

$$
\frac{d \theta}{d x}=\frac{\theta}{x}
$$

Hence, the locus of these points, called the G-curve, is given by

$$
G(x, y)=y U_{x}-x U_{y}=0 .
$$

$G$-curves also pass through the origin and are symmetric with respect to it. Obviously, $F$ and $G$-curves have the slope $\theta_{0}{ }^{\prime}$ at the origin.

Now, transversals are seen from (7.13) to be themselves symmetric with respect to the origin. Hence, the field of transversals looks as shown in Figure 6. In that diagram, $P_{0}\left(X_{0}, Y_{0}\right)$ is the point where the straight line, passing with slope $\theta_{0}^{\prime}$ through the origin, intersects the $L_{a}$-curve. $P_{1}\left(X_{1}, Y_{1}\right)$ is that where the $G$-curve intersect the $L_{a}$-curve, and $P_{2}\left(X_{2}, Y_{2}\right)$ is that where the $F$-curve intersects the $L_{a}$-curve.

We show now, that at least one $F$-curve and $G$-curve always exist and intersect the $L_{a}$-curve. Since every equipotential curve has, in the first quadrant for instance, always at least one point of stationary distance

- It can happen that $h(\lambda)$ has four real roots when $k=3$. This has been shown to be an exceptional case and is not treated here. [85]. 
from the origin, that quadrant contains at least one $G$-curve. Consider now the transversal issuing from $P_{1}$. It points initially towards the origin. If $P_{0}$ and $P_{1}$ do not coincide, this particular transversal must have an inflection point prior to its arrival at the origin with slope $\theta_{0}{ }^{\prime}$. In that case, the transversal issuing from $P_{0}$ points initially above the origin, and

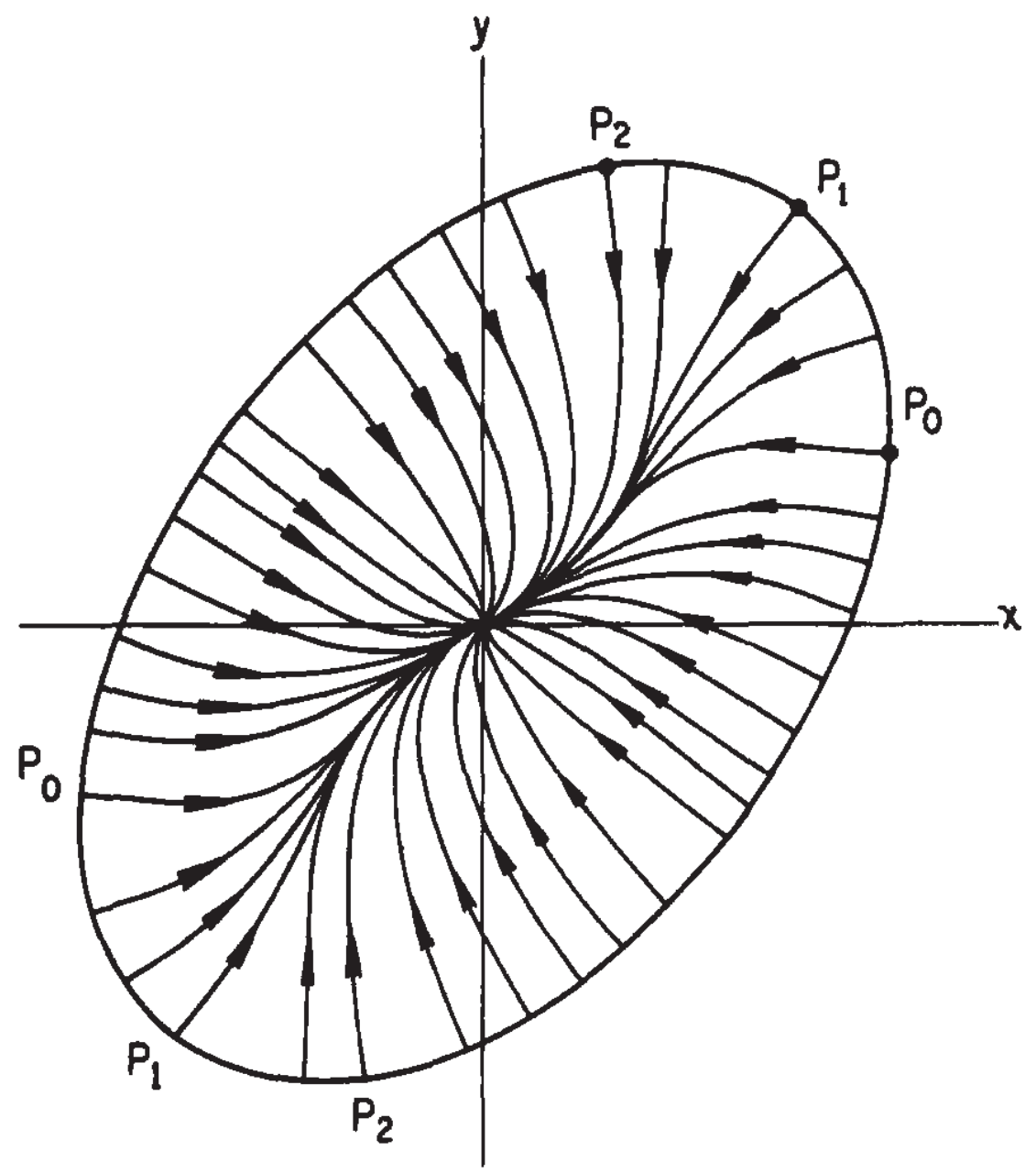

FIG. 6. The directed transversals in nonlinear system with two degree of freedom.

it must also have a point of inflection before arriving with slope $\theta_{0}^{\prime}$ at the origin. Hence, in general, there exist transversals with points of inflection, and there exists at least one $F$-curve in the first quadrant. The transversals will be endowed with a sense of direction by considering the points $P(X, Y)$ which compose the $L_{a}$-curve as their points of issue. These directed transversals in Figure 6 are supplied with arrow heads pointing toward the origin. In this way, the transversals give not only the direction, but also the sense, of the forces acting on the unit mass point of the pseudo-system.

\section{The T-curves [19]}

The trajectories corresponding to motions (at energy level $h$ ) of admissible, autonomous systems fall into two general classes: those which attain the $L_{a}$-surface, and those which do not. By definition, $T$-curves are those integral curves of (3.12) which do intercept the $L_{a}$-surface. Hence, the $T$-curves constitute the class of all trajectories which correspond to motion with a 
rest-point of admissible, autonomous systems. Note that $T$-curves need not be admissible in the sense of Section V.

We shall establish here certain general properties of $T$-curves of systems having two degrees of freedom. All $T$-curves intercept the $L_{a}$-curve, and all $P$-curves intersect it. The $P$-curve which issues from the same point $P(X, Y)$ from which a $T$-curve issues will be called "the transversal associated with that $T$-curve," or simply the associated $P$-curve.

Some of the properties of $T$-curves are easily established by means of the equation of the trajectories (7.6), written in the form

$$
y^{\prime \prime}=-\frac{\left(1+y^{\prime 2}\right)}{2(U+h)} U_{x}\left(y^{\prime}-\theta^{\prime}\right)
$$

it results immediately from a substitution of (7.13) in (7.6).

Because of the definition of $P$-curves and $T$-curves and Theorem $\mathrm{V}-\mathrm{V}$, one has:

Property 1: Every $T$-curve is tangent to its associated $P$-curve on the $L_{a}$-curve.

Less obvious is:

Property 2: The curvature $\kappa_{T}(X, Y)$ of a $T$-curve at $P(X, Y)$ has the same sign, but is less in magnitude than the curvature $\kappa_{P}(X, Y)$ of its associated $P$-curve.

To prove it, one observes that the second derivative $y^{\prime \prime}$ in (7.6) is indeterminate on the $L_{a}$-curve. Evaluating it by l'Hospital's rule

$$
y^{\prime \prime}(X, Y)=\frac{1}{3}\left[\frac{U_{x y}+y^{\prime}\left(U_{y y}-U_{x x}-y^{\prime} U_{x y}\right)}{U_{x}}\right]_{X, Y}
$$

But, by Property 1 and (7.13),

$$
y^{\prime}(X, Y)=\theta^{\prime}(X, Y)=U_{y}(X, Y) / U_{x}(X, Y) .
$$

Consequently,

$$
y^{\prime \prime}(X, Y)=\frac{1}{3}\left[\frac{U_{x y}\left(U_{x}{ }^{2}-U_{y}{ }^{2}\right)-U_{x} U_{y}\left(U_{x x}-U_{y y}\right)}{U_{x}^{3}}\right]_{X, Y},
$$

and, comparing (7.38) and (7.45)

$$
\kappa_{T}(X, Y)=\frac{1}{8} \kappa_{P}(X, Y),
$$

which proves Property 2.

Next, we have:

Property 3: A T-curve which is not a straight line cannot coincide everywhere with its associated $P$-curve.

The proof is evident from (7.42). From that equation, one can also deduce immediately: 
Property 4: If any trajectory (and, hence, any $T$-curve) is tangent to a $P$-curve at any regular point, the trajectory has zero curvature at that point.

This property of trajectories was earlier noted by Kauderer [10].

Property 5: At any regular point, every curved trajectory presents its convex side to the incoming, directed $P$-curves.

This property is a consequence of Theorem VII-I. It merely states that the trajectory of the unit mass point is being turned, by the forces acting on it, in such a direction as to yield to these forces.

Property 6: Every $T$-curve that intersects its associated $P$-curve at a regular point $Q(x, y)$, must have passed through a point of zero curvature on the arc between $P(X, Y)$ and the point of intersection $Q(x, y)$. This property follows from the observation that the $P$-curves constitute a regular field, and the $T$-curves are smooth. Hence, there exists a point on the arc lying between $P(X, Y)$ and $Q(x, y)$ where the $T$-curve is tangent to some $P$-curve. By Property 4, this is a point of zero curvature of the $T$-curve.

Finally, we have:

Property 7: Every trajectory (and, hence, every $T$-curve) either passes through the origin of the $x y$-plane or, else it must have at least one point of tangency with at least one $E$-curve (see the appendix).

To prove it, we observe that the $E$-curves constitute a regular field of simple, closed curves surrounding the origin. Moreover, trajectories are smooth, and by Theorem V-II, they are confined to the closed domain $D_{a}$. It follows from these observations that Property 7 is true.

It is clear that one may consider the origin as the (degenerate) equipotential line of zero energy. In that case, Property 7 might be interpreted as stating that every $T$-curve is tangent at least once to an $E$-curve, and $T$-curves which are tangent to the $E$-curve of zero energy are necessarily simple trajectories and, perhaps, modal lines.

It seems probable, that the above properties of $T$-curves could also be established for systems whose number of degrees of freedom exceeds two.

\section{Special Autonomous Systems}

\section{Smooth Systems}

It is evident from the equations of motion (3.3) of the pseudo-system that all differences between given, autonomous systems consist in differences between their potential functions.

An autonomous system is said to be smooth if its potential function is of the form

$$
0=-\sum_{i=1}^{n} \sum_{j=i+1}^{n} \sum_{k=8,5, \ldots}^{s_{i j}} \frac{a_{i j}^{(k)}}{k+1}\left(\frac{u_{i}}{\sqrt{m_{i}}}-\frac{u_{j}}{\sqrt{m_{j}}}\right)^{k+1}
$$


where the $a_{i j}^{(k)}$ are constants. Then, the spring force of a spring $S_{i j}$ between the masses $m_{i}$ and $m_{j}$ is

$$
\frac{d \widehat{O}_{i j}}{d w}=-\sum_{k=1,3, \ldots}^{r_{i j}} a_{i j}^{(k)} w^{k}
$$

where

$$
w=u_{i}-u_{i}
$$

is the length-change. Hence, all derivatives of every spring force with respect to the length-change exist, which is the reason for the terminology "smooth". While all spring forces of smooth systems are assumed to be finite polynomials, it is not required that these polynomials be all of the same degree. Thus, while the restriction to smooth systems may appear severe from the mathematical point of view, it is mild from the standpoint of physics.

\section{Uniform Systems [9]}

A system is said to be uniform if its potential function is of the form [9]

$$
U=-\sum_{m=1, \mathrm{~s}, \ldots} \frac{a^{(m)}}{m+1} \sum_{i=1}^{n} \sum_{i=i+1}^{n}\left(x_{i}-x_{j}\right)^{m+1} .
$$

One sees from (8.4) that uniform systems are those in which all springs are equal, and all masses are equal (in which case one may, without loss of generality, put the masses equal to unity).

For uniform systems, one can readily demonstrate an interesting and useful property. Let us denote as the potential function of the associated, linear system that whose potential function is [9]

$$
\tilde{O}=-\frac{a^{(1)}}{2} \sum_{i=1}^{n} \sum_{j=i+1}^{n}\left(x_{i}-x_{j}\right)^{2}
$$

where the values of $a^{(1)}$ are the same in (8.4) and (8.5). Then, one has

Theorem VIII-I: The modal lines of an autonomous, uniform system are straight. Their directions coincide with those of the associated linear system.

One proof of this theorem is due to Mawhin [6] who shows that the modal lines of (8.4) are straight, and that their direction is independent of $m$. The importance of this theorem is evident. The modal lines, when $U=\tilde{U}$, are the principal axes of the $n$-dimensional ellipsoids

$$
\tilde{U}+h^{*}=0, \quad\left(0<h^{*} \leqslant h\right)
$$


and the directions of these axes are readily found by means of linear eigenvalue theory. The time-history of the motions can, then, be found subsequently by simple quadratures.

The geometrical interpretation of this result is that the equations

$$
U+h^{*}=0, \quad\left(0<h^{*} \leqslant h\right)
$$

are ovaloids which are "distorted ellipsoids." However, these distortions are such that the ovaloids have the same symmetries with respect to the modal lines as the ellipsoids of (8.6).

\section{Sequential Anchored Systems}

With Haughton [27], we call a system sequential if it consists of a chain of $\boldsymbol{n}$ finite masses in which each mass is coupled by a "smooth" spring (as above) to only its neighbors. It is said to be anchored if the first and $n$th masses are also coupled to a fixed point by "smooth" springs. This is in fact, the model that is usually employed in the theory of linear vibrations of multi-degree-of-freedom systems. The potential function of anchored, sequential systems is of the form

$$
\begin{aligned}
0= & -\sum_{k=1,3, \ldots}\left\{\frac{a_{1}^{(k)}}{k+1}\left(\frac{u_{1}}{\sqrt{m_{1}}}\right)^{k+1}+\frac{a_{n}{ }^{(k)}}{k+1}\left(\frac{u_{n}}{\sqrt{m_{n}}}\right)^{k+1}\right. \\
& \left.+\sum_{i=1}^{n} \frac{a_{i j}^{(k)}}{k+1}\left(\frac{u_{i}}{\sqrt{m_{i}}}-\frac{u_{j}}{\sqrt{m_{j}}}\right)^{k+1}\right\}
\end{aligned}
$$

where $j=i+1$.

Haughton [27], using methods similar to those of Mawhin [6] proves the following remarkable

Theorem VIII-II: If an admissible, anchored, sequential system has straight modal lines, their directions are the same as those of the associated, linear system of potential function

$$
\tilde{U}=-\left\{\frac{a_{1}^{(2)}}{2}\left(\frac{u_{1}}{\sqrt{m_{1}}}\right)^{2}+\frac{a_{n}^{(2)}}{2}\left(\frac{u_{n}}{\sqrt{m_{n}}}\right)^{2}+\sum_{i=1}^{n-1} \frac{a_{i j}^{(2)}}{2}\left(\frac{u_{i}}{\sqrt{m_{i}}}-\frac{u_{i}}{\sqrt{m_{i}}}\right)^{2}\right\}, \quad(j=i+1) .
$$

If, in an anchored, sequential system, all masses are equal and all springs are equal it is said to be anchored, sequential and uniform. In that case, one can readily show that straight modal lines do, indeed, exist; the problem of normal mode vibrations can, then, be completely solved by considering a linear system and by performing one additional quadrature. 


\section{Homogeneous Systems [28]}

An admissible, autonomous system is said to be homogeneous if its potential function is of the form

$$
O=-\sum_{i=1}^{n} \sum_{j=i+1}^{n} \frac{a_{i j}^{(h)}}{k+1}\left|\frac{x_{i}}{\sqrt{m_{i}}}-\frac{x_{j}}{\sqrt{m_{j}}}\right|^{n+1}
$$

where $k$ is a real number in $0<k<\infty$. It derives its name from the fact that the potential function is homogeneous in the $x_{i}$ of degree $k+1$.

It is interesting to form the expression for the spring forces. These forces are given by

$$
\frac{\partial 0}{\partial u_{i}}=-\sum_{j=1}^{n} \frac{a_{i j}^{(k)}}{\sqrt{m_{i}}}\left(u_{i}-u_{j}\right)\left|u_{i}-u_{j}\right|^{k-1}, \quad(i=1, \ldots, n) .
$$

This equation shows that the spring forces are odd functions, and proportional to the $k$ th power, of the length-changes. Clearly, when one puts $k=1$, one recovers the linear system.

Physically, neither masses nor springs need be equal in homogeneous systems. Instead, the springs are nonlinear "in the same way"; i.e., all obey the same simple power law.

It is not difficult to construct a physical system for which $k=3$ [21]. Consider an arrangement of masses and springs as in Figure 1. However, in the present case, the translations $u_{i}$ are not in the direction of the chain, but normal to it. Moreover, every spring is assumed linear and its free length is exactly equal to the distance between the masses interconnected by that spring when the system is in the equilibrium configuration. Clearly, the spring forces are always restoring, or $\bar{O}$ is negative, definite; moreover, the spring forces reverse sign when the displacements do, or 0 is symmetric with respect to the origin. However, infinitesimal displacements $u_{i}$ which are small of the first order produce length-changes in the springs which are small of the second order. Hence, for sufficiently small displacements, $k=3$.

By applying the criterion (6.3) one can readily prove [9]

Theorem VIII-III: The modal lines of admissible, autonomous, homogeneous systems are straight. Their direction is defined by the systems of roots of the transcendental equations (6.4).

\section{Symmetric Systems}

With Mawhin [6] we call a system symmetric if its potential function is of the form 


$$
\begin{aligned}
0= & -\sum_{j=1}^{n} \sum_{m=1,3, \ldots}^{r_{a}} \frac{a_{0}^{(m)}}{m+1} x_{j}^{m+1}-\sum_{j=1}^{n} \sum_{m=1,3, \ldots}^{r_{a}} \frac{a_{m+1}^{(m)}}{m+1} x_{j}^{m+1} \\
& -\sum_{i=1}^{n} \sum_{j=i+1}^{n} \sum_{m=1,3, \ldots}^{r_{c}} \frac{a_{i j}^{(m)}}{m+1}\left(x_{i}-x_{i}\right)^{m+1} .
\end{aligned}
$$

The physical properties of symmetric systems are the following: All masses are equal (hence, assumed equal to unity, without loss of generality), all spring forces are smooth (in the above sense), all anchor springs are equal, and the spring forces in all coupling springs are polynomials of the same degree. This is a generalization of the "symmetric two-degree-of-freedom system" [29] which was called "symmetric" because the system had literally physical symmetry about its centerline. (It should be noted that Mawhin denotes the spring between $m_{0}$ and $m_{1}$ as $S_{0}$, that between $m_{0}$ and $m_{j}$ as $S_{j}$, and that between $m_{0}$ and $m_{n}$ as $S_{n}$.)

If a symmetric system has only two degrees of freedom, one can readily show [29] that straight modal lines exist, and they have the same inclination as those of the associated linear system.

\section{The ateb-Functions [20]}

\section{Their Origin and Importance}

As the name indicates, the ateb-functions are inversions of certain beta functions, much in the same way as elliptic functions are inversions of elliptic integrals. Similar to elliptic functions which satisfy the autonomous Duffing equation, the ateb-functions solve the problem of normal-mode vibrations of homogeneous systems. In common with elliptic functions, the ateb-functions depend on a parameter $n$ as well as an argument. It is interesting to note that Gauss [30] was the first to invert a betafunction for the case $n=2$. His problem arose in the rectification of the lemniscate, and his lemniscate functions were, in fact, the first functions to be defined by the inversion of an integral. Later, Legendre showed [30] that the period of the lemniscate functions was expressible in terms of gamma functions-an observation that has been repeated independently by many authors in recent times $[31,32,10,33]$.

We have shown that the modal lines of homogeneous systems are straight. Hence, they are expressible in the form

$$
x_{i}=c_{i p} x_{1}, \quad(i=2, \ldots, n ; \quad p=1, \ldots, m)
$$

where $m$ is the number of modal lines. If one substitutes (9.1) into the first of the equations of motion

$$
\ddot{x}_{1}=-\sum_{i=1}^{n} \frac{a_{1 j}^{(k)}}{\sqrt{m_{1}}}\left(\frac{x_{1}}{\sqrt{m_{1}}}-\frac{x_{j}}{\sqrt{m_{j}}}\right)\left|\frac{x_{1}}{\sqrt{m_{1}}}-\frac{x_{j}}{\sqrt{m_{j}}}\right|^{k-1}, \quad(0<k<\infty)
$$


of a homogeneous system, one finds

$$
\ddot{x}_{1}+C_{p} x_{1}\left|x_{1}\right|^{k-1}=0, \quad(p=1, \ldots, m)
$$

where

$$
\begin{aligned}
& C_{p}=m_{1}-(k+1) / 2\left[\sum_{j=1}^{n} a_{1 j}^{(k)}\left(1-c_{j p} \mu_{j}\right)\left|1-c_{i p} \mu_{j}\right|^{k-1}\right], \\
& \mu_{j}^{2}=m_{j} / m_{1} .
\end{aligned}
$$

We shall show that (9.3) has periodic solutions of period $T_{p}$, and it follows from (9.1) that, then all $x_{i}(t),(i=2, \ldots, n)$ are also periodic of the same period. Hence, the resulting motion is a normal mode vibration.

We shall integrate $(\mathbf{9 . 3})$ for the Cases I and II where, for

$$
\begin{array}{llll}
\text { Case I and II: } & \dot{x}_{1}=0 \text { when } & x_{1}=X_{1}>0, \\
\text { Case I: } & x_{1}=0 \text { when } t=0, \\
\text { Case II: } & x_{1}=X_{1} \text { when } t=0 .
\end{array}
$$

The conditions for Case II are initial conditions because $x_{1}$ and $\dot{x}_{1}$ are specified at the same instant of time. However, in Case I the velocity and displacement are prescribed at different instants of time. Hence, in Case I, it must be shown a posteriori that, if $x_{1}(0)=0$, then there exists an $\dot{x}_{1}(0)$ such that $x_{1}\left(t_{0}\right)=X_{1},\left(X_{1}, t_{0}>0\right)$.

First, we change (9.3) into the canonical form by means of the transformations [20]

$$
\tau=\left(C_{p} / n\right)^{1 / 2} X_{1}^{n-1} t ; \quad x_{1}=\xi X_{1} ; \quad n=(k+1) / 2
$$

and, because of the definition of $n$ and the bounds on $k$,

$$
n=1 \text { when } k=1 ; \quad 1 / 2<n<\infty .
$$

Under (9.6), the equation (9.3) goes over into

$$
\xi^{\prime \prime}+n \xi|\xi|^{2(n-1)}=0, \quad\left({ }^{\prime}=\frac{d}{d \tau}\right)
$$

and equations (9.5) become for

$$
\begin{array}{llll}
\text { Case I and II: } & \xi^{\prime}=0 & \text { when } & \xi=1, \\
\text { Case I: } & \xi=0 & \text { when } & \tau=0, \\
\text { Case II: } & \xi=1 & \text { when } & \tau=0 .
\end{array}
$$


A first integral of (9.8) satisfying the first of (9.9) is

$$
\xi^{\prime}= \pm\left(1-|\xi|^{2 n}\right)^{1 / 2}
$$

and a second integral becomes, for Case I

$$
\tau= \pm \int_{0}^{\xi}\left(1-|r|^{2 x}\right)^{-1 / 2} d r
$$

and for Case II

$$
\tau= \pm \int_{1}^{\xi}\left(1-|r|^{2 n}\right)^{-1 / 2} d r= \pm\left\{\int_{0}^{\xi}\left(1-|r|^{2 n}\right)^{-1 / 2} d r-\int_{0}^{1}\left(1-|r|^{2 n}\right)^{-1 / 2} d r\right\},
$$

with $0 \leqslant \xi \leqslant 1$. The sign ambiguities in (9.11) and (9.12) are readily resolved with the result that the + sign must be chosen in (9.11) and the - sign in (9.12) [20].

The question whether there exists a real $\tau_{0}>0$ such that $\xi\left(\tau_{0}\right)=1$ is now easily answered by observing that (9.11) exists when $\xi=1$. In fact, as observed by many authors $[31,32,10,33]$ under the change of variable

$$
r^{2 n}=s
$$

the integral (9.11) with upper limit $\xi=1$ becomes

$$
\tau(1)=\frac{1}{2 n} \int_{0}^{1} s^{(1-2 n) / 2 n}(1-s)^{-1 / 2} d s=\frac{1}{2 n} B\left(\frac{1}{2 n}, \frac{1}{2}\right)
$$

where $B\left(1 /(2 n), \frac{1}{2}\right)$ is the complete beta function $B(p, q)$ of parameters $p=1 / 2 n$, $q=\frac{1}{2}$. Similarly,

$$
\tau(\xi)=\frac{1}{2 n} B_{\xi *}\left(\frac{1}{2 n}, \frac{1}{2}\right), \quad \xi^{*}=\xi^{1 / 2 n}
$$

where $B_{\xi^{*}}(p, q)$ is the incomplete beta function ${ }^{\dagger}$ of argument $\xi^{*}$ and parameters $p=1 / 2 n, q=\frac{1}{2}$.

† We use here Pearson's notation [84]. 


\section{The Inversions}

From the above results it follows that the solutions of (9.8) are, for Case I

$$
\tau=\frac{1}{2 n} B_{*}\left(\frac{1}{2 n}, \frac{1}{2}\right)
$$

and for Case II

$$
\tau=\frac{1}{2 n}\left[B\left(\frac{1}{2 n}, \frac{1}{2}\right)-B_{*}\left(\frac{1}{2 n}, \frac{1}{2}\right)\right]
$$

with

$$
\xi^{*}=\xi^{1 / 2 n} \text {. }
$$

But, if $\xi(\tau)$ is periodic, the solutions (9.16) and (9.17) cannot be singlevalued on an interval exceeding one-half period. Hence, it is desirable to invert these solutions. The definitions used in the inversions, and the transformations are summarized in the table below. Cumbersome sign distinction are avoided if " $n$ is regarded to behave like an odd integer." This phrase means that negative quantities, raised to the power $n+p$, where $p$ is either zero or an even integer, remain negative, and negative quantities, raised to the power $n+q$ where $q$ is an odd integer, become positive. The absolute values of these powers are given by

$$
a^{n+p}=|a|^{n+p}
$$

with a similar expression for powers $n+q$.

$$
\begin{array}{c|c}
\text { Case I } & \text { Case II } \\
u_{1}=\int_{0}^{\xi_{2}}\left(1-|r|^{2 n}\right)^{-1 / 2} d r=F_{1}\left(\xi_{1}\right) & u_{2}=-\int_{1}^{\xi_{1}}(1-|r| 2 n)^{-1 / 2} d r=F_{2}\left(\xi_{2}\right) \\
\xi_{1}=\sin ^{1 / n} \varphi_{1} ; \quad r=\sin ^{1 / n} \theta & \xi_{8}=\cos ^{1 / n} \varphi_{2} ; \quad r=\cos ^{1 / n} \theta \\
m u_{1}=\int_{0}^{\varphi_{1}} \sin ^{(1-n) / n} \theta d \theta=G_{1}\left(\varphi_{1}\right) & n u_{2}=\int_{0}^{\varphi_{2}} \cos ^{(1-n) / n} \theta d \theta=G_{2}\left(\varphi_{2}\right) \\
\varphi_{1}=\operatorname{amp} n u_{1} & \varphi_{2}=\operatorname{amp} n u_{2} \\
\xi_{1}=\sin 1 / n\left(\operatorname{amp} n u_{1}\right)=\operatorname{sam}\left(n u_{1}\right) & \xi_{2}=\cos ^{1 / n}\left(\operatorname{amp} n u_{2}\right)=\operatorname{cam}\left(n u_{2}\right)
\end{array}
$$




$$
0 \leqslant \xi_{1}, \xi_{2} \leqslant 1 ; \quad 0 \leqslant \varphi_{1}, \varphi_{2} \leqslant \frac{\pi}{2}
$$

It is seen that the inversions lead to four ateb-functions: Those called amp $n u_{1,2}$ are inversions of $G_{1,2}\left(\varphi_{1,2}\right)$, where $G_{1,2}$ are beta functions, and those called $\operatorname{sam}\left(n u_{1}\right)$ and cam $\left(n u_{2}\right)$ are respectively the inversions of $F_{1,2}\left(\xi_{1,2}\right)$ which are also beta functions.

A certain number of properties of ateb-functions have been determined [20] and are summarized below.

\section{The Amp-Functions}

The amp-functions are odd, or

$$
\operatorname{amp}-n u_{1,2}=-\operatorname{amp} n u_{1,2}
$$

When $n=1$,

$$
\operatorname{amp} u_{1,2}=u_{1,2}
$$

Particular values are given by

$$
\operatorname{amp} n u^{*}=\pi / 2
$$

$$
\operatorname{amp} \mathbf{0}=\mathbf{0} \text {, }
$$

where

$$
u^{*}=u_{1}^{*}=u_{2}^{*}=\frac{1}{2 n} B\left(\frac{1}{2 n}, \frac{1}{2}\right)
$$

They satisfy the relation (also satisfied by the elliptic am-function)

$$
\operatorname{amp} n\left(p u^{*} \pm u_{1,2}\right)=p \frac{\pi}{2} \pm \operatorname{amp} n u_{1,2}
$$

where $p$ is a positive odd integer.

Their first derivatives are

$$
\frac{d}{d u_{1}}\left(\operatorname{amp} m u_{1}\right)=n \operatorname{sam}^{n-1}\left(n u_{1}\right),
$$

$$
\frac{d}{d u_{2}}\left(\operatorname{amp} n u_{2}\right)=n \operatorname{cam}^{n-1}\left(n u_{2}\right) .
$$


Special values of these derivatives are

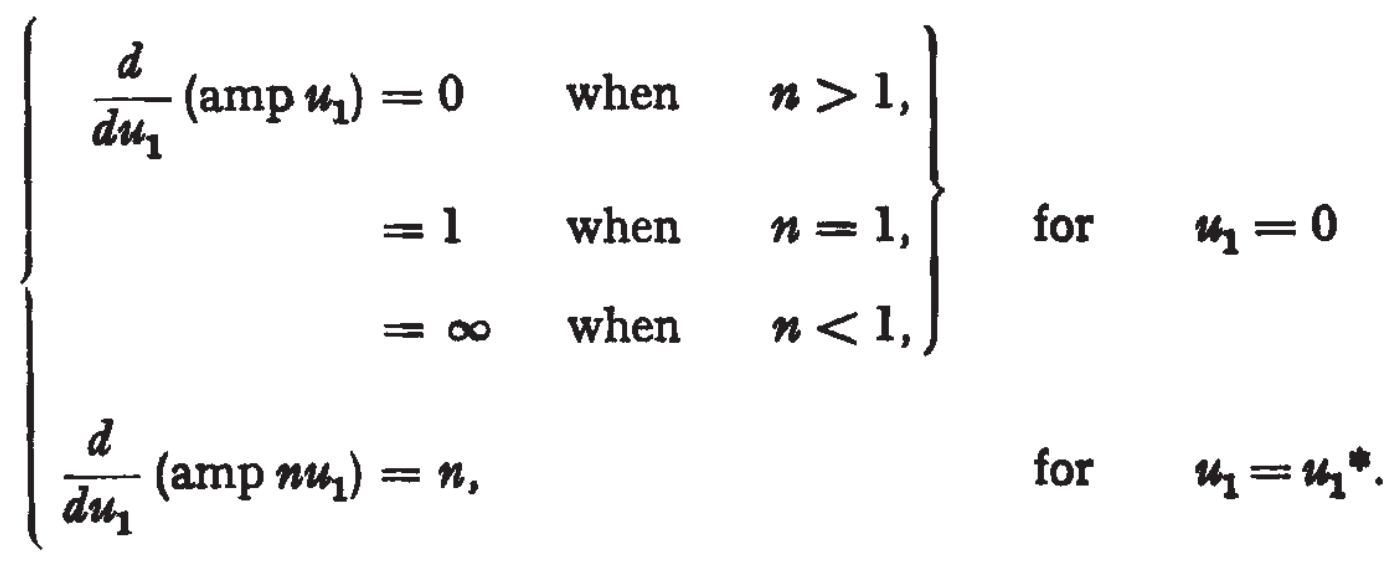

(9.26)

(9.27)

$$
\left\{\begin{array}{rlrl}
\frac{d}{d u_{2}}\left(\operatorname{amp} n u_{2}\right) & =0, & & \text { for } \quad u_{2}=0 \\
\frac{d}{d u_{2}}\left(\operatorname{amp} n u_{2}\right) & =0 \quad \text { when } \quad n>1, \\
& =1 \quad \text { when } \quad n=1, \\
& =\infty \quad \text { when } \quad n<1 .
\end{array}\right\} \text { for } \quad u_{2}=u_{2}^{*}
$$

"amp"-functions look as shown in Figure 7(a) and (b).

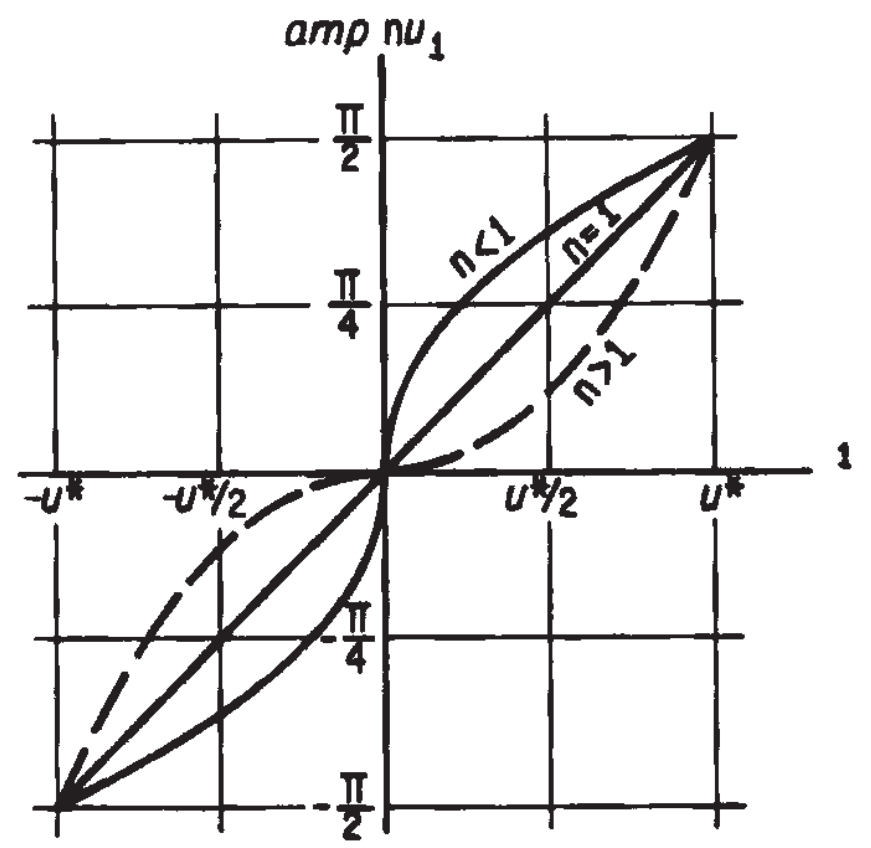

(a)

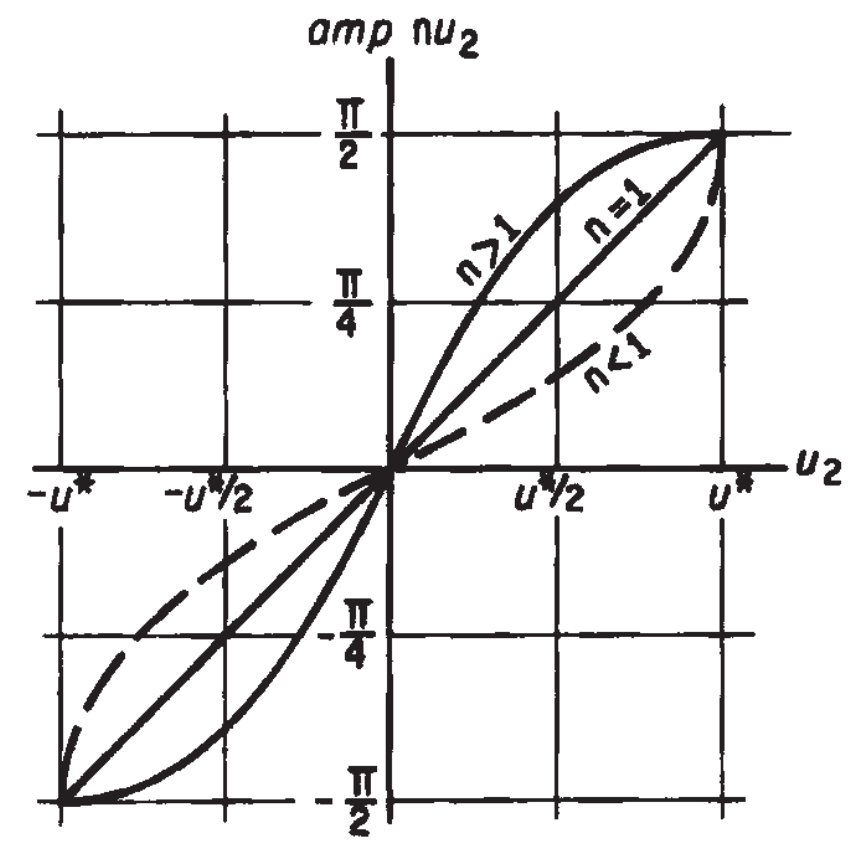

(b)

FIG. 7. The amp-functions. 


\section{The Sam- and Cam-Functions}

The sam- and cam-functions are periodic of the same period; i.e.,

$$
\begin{aligned}
& \operatorname{sam}\left(n u_{1}\right)=\operatorname{sam}\left(n u_{1}+4 n u^{*}\right), \\
& \operatorname{cam}\left(n u_{2}\right)=\operatorname{cam}\left(n u_{2}+4 n u^{*}\right),
\end{aligned}
$$

where $u^{*}$ is defined in $(9.23)$.

One is odd, the other even, or

$$
\begin{aligned}
& \operatorname{sam}\left(-n u_{1}\right)=-\operatorname{sam}\left(n u_{1}\right), \\
& \operatorname{cam}\left(-n u_{2}\right)=\operatorname{cam}\left(n u_{2}\right) .
\end{aligned}
$$

When $n=1$, they become circular functions, and when $n=2$, they become elliptic functions, or

(9.30)

$$
\begin{aligned}
\operatorname{sam}\left(u_{1}\right) & =\sin u_{1}, \\
\operatorname{cam}\left(u_{2}\right) & =\cos u_{2}, \\
\operatorname{sam}\left(2 u_{1}\right) & =\operatorname{sn}\left(2^{-1 / 2}, u_{1}\right), \\
\operatorname{cam}\left(2 u_{2}\right) & =\operatorname{cn}\left(2^{-1 / 2}, u_{2}\right) .
\end{aligned}
$$

If $u_{1}{ }^{0}$ and $u_{2}{ }^{0}$ are those values of $u_{1}$ and $u_{2}$ for which amp $n u_{1}^{0}=$ amp $n u_{2}{ }^{0}$,

$$
\operatorname{sam}^{2 n}\left(n u_{1}^{0}\right)+\operatorname{cam}^{2 n}\left(n u_{2}^{0}\right)=1 \text {, }
$$

thus generalizing a well-known identity of trigonometric functions. The derivatives of these functions are

(9.32)

(9.33)

$$
\begin{aligned}
& \left\{\begin{array}{l}
\frac{d}{d u_{1}^{0}}\left[\operatorname{sam}\left(n u_{1}^{0}\right)\right]=\operatorname{cam}^{n}\left(n u_{2}^{0}\right), \\
\frac{d}{d u_{2}^{0}}\left[\operatorname{cam}\left(n u_{2}^{0}\right)\right]=-\operatorname{sam}^{n}\left(n u_{1}^{0}\right), \\
\frac{d^{2}}{d u_{1}^{2}}\left[\operatorname{sam}\left(n u_{1}\right)\right]=-n \operatorname{sam}^{2 n-1}\left(n u_{1}\right), \\
\frac{d^{2}}{d u_{2}^{2}}\left[\operatorname{cam}\left(n u_{2}\right)\right]=-n \operatorname{cam}^{2 n-1}\left(n u_{2}\right) .
\end{array}\right.
\end{aligned}
$$

The sam- and cam-functions look as shown in Figure 8 and Figure 9 for a variety of values of $n$ in $\frac{1}{2} \leqslant n \leqslant \infty$. 


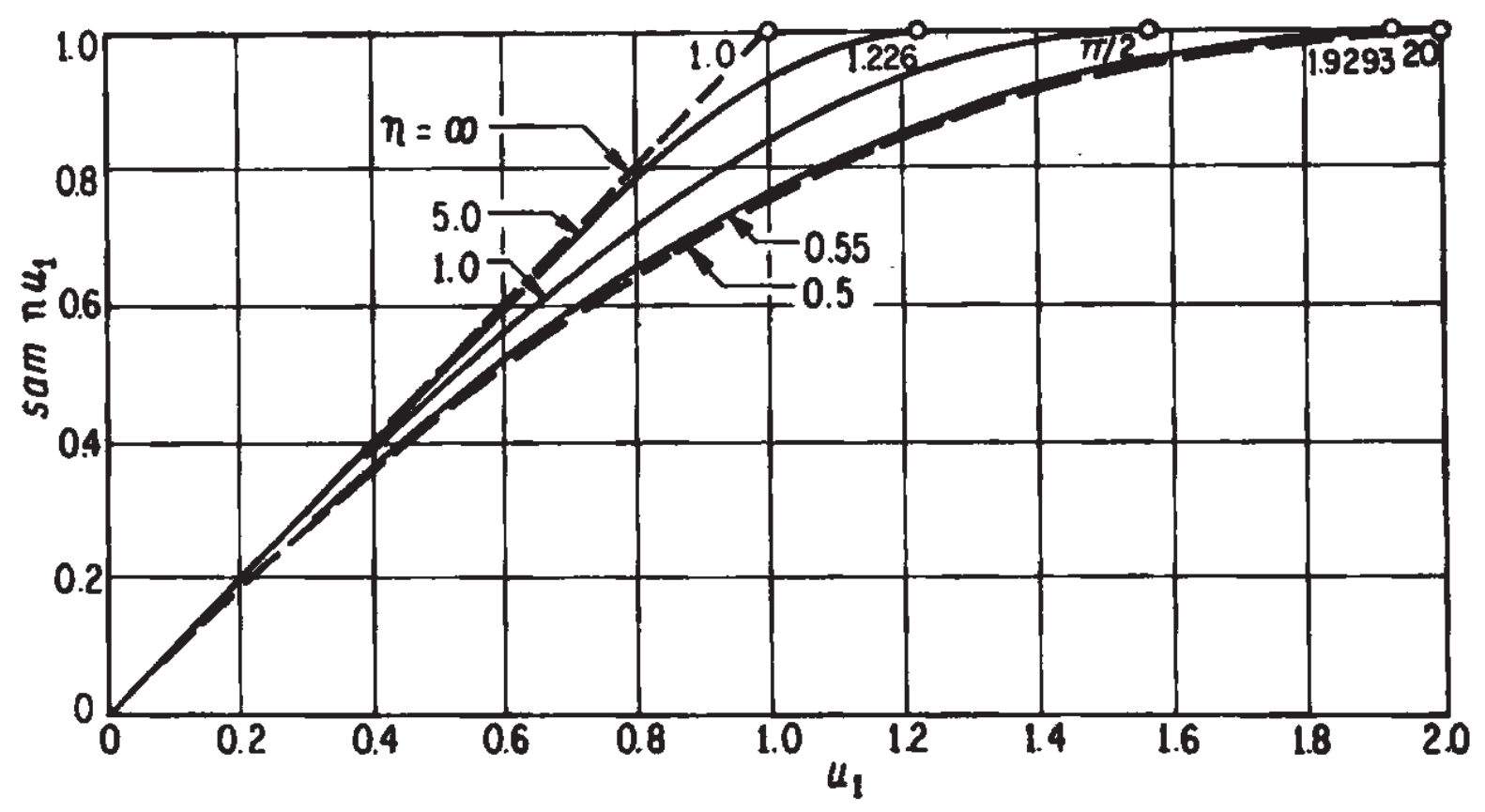

Fig. 8. The sam-function.

For $n=1$, the curves are the trigonometric sine and cosine curve, respectively. For $n=\frac{1}{2}$, they are parabolas, and for $n=\infty$, they consist of segments of straight lines.

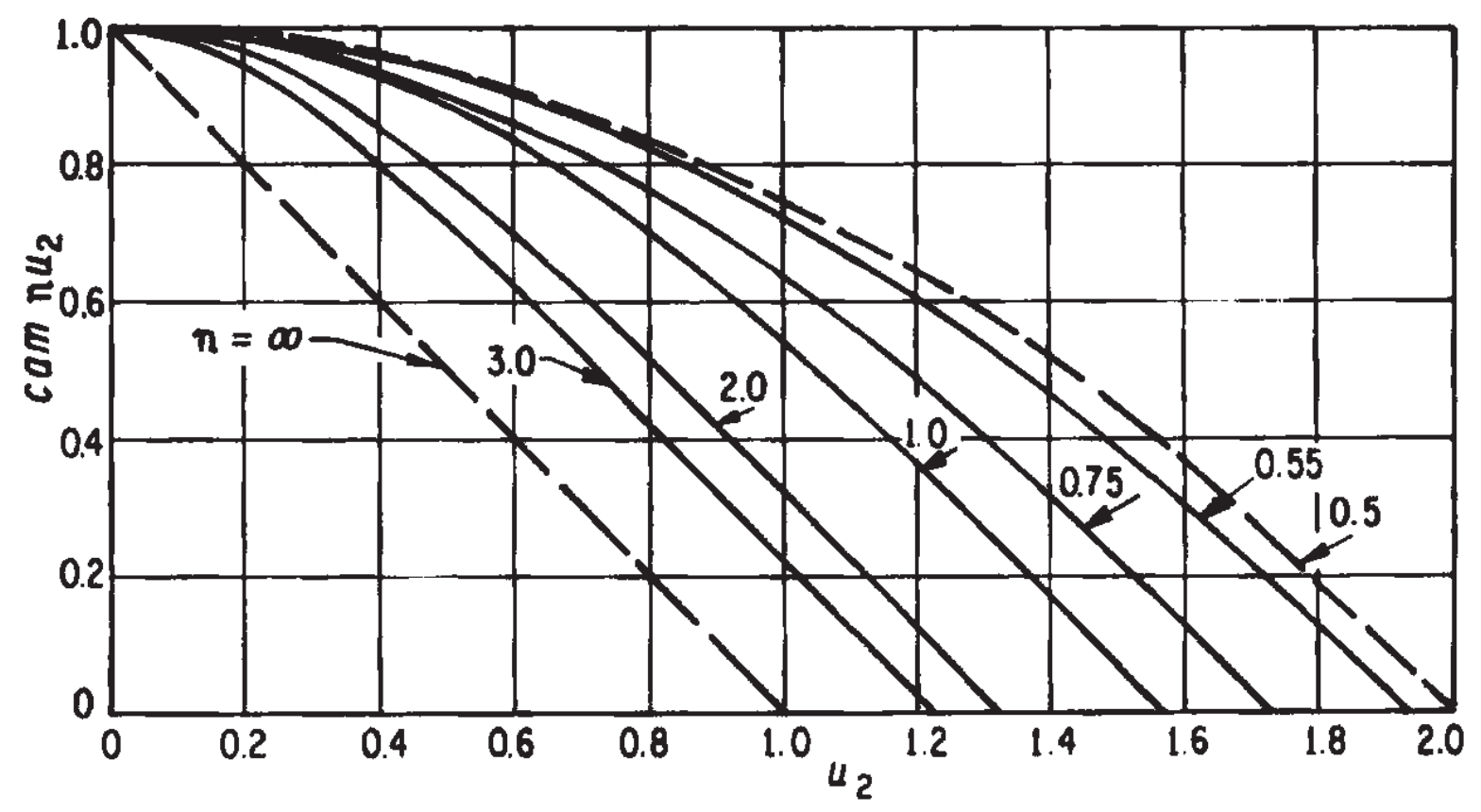

Fig. 9. The cam-function.

It is evident that the solutions of (9.8) are for Case I and II, respectively,

$$
\begin{aligned}
& \xi=\operatorname{sam}(n \tau), \\
& \xi=\operatorname{cam}(n \tau),
\end{aligned}
$$

as one can readily verify by direct substitutions, and by making use of (9.33). 


\section{The Perturbation Potential}

The trajectories of nonsimilar normal-mode vibrations of autonomous, admissible systems are strictly homogeneous curves passing through the origin of the configuration space and intercepting the $L_{a}$-surface, and they are integral curves of the system $M$ defined in (3.12).

At present, there exists no hope of finding the integrals of $M$ in the general case, considering the nonlinear character of the system (3.12). However, as shown above, there exist many strongly nonlinear systems whose modal lines are straight. Systems "neighboring on these" may have curved modal lines; hence, their normal-mode vibrations may be nonsimilar. Here, we shall show how such modal lines may be found by perturbation methods. The results, reported here, are due to Kuo [18]. They are generalizations of the results found earlier in the case of two degrees of freedom [8]. Let

$$
U_{0}=U_{0}\left(x_{1}, \ldots, x_{n} ; m_{1}, \ldots, m_{n} ; a_{1}, \ldots, a_{l}\right)
$$

be the admissible potential function of an autonomous system having at least one straight modal line, defined by

$$
x_{i}^{*}=c_{i} x_{1}, \quad(i=2, \ldots, n) .
$$

In $(10.1)$, the $m_{i},(i=1, \ldots, n)$ are the $n$ masses and the $a_{j},(j=1, \ldots, l)$ are parameters defining the properties of the springs.

We shall examine systems whose potential function neighbors on $U_{0}$; hence, we call $U_{0}$ the potential function of the parent system.

We shall say, "a system neighbors on the parent system" if its potential function is of the form

$$
\hat{O}=U_{0}+\varepsilon \omega_{0}, \quad|\varepsilon| \ll\left|U_{0}\right|,
$$

where $O$ is admissible; consequently, $\omega_{0}$ must also satisfy the admissibility conditions of potential functions. We call $\bar{O}$ the potential function of the perturbed system, and $\omega_{0}$ the perturbation potential. It is evident from (10.1) that the $m_{i}$ and $a_{i}$ are the only parameters subject to perturbations. Therefore, the most general form of the perturbation potential is

$$
\omega_{0}=\sum_{i=1}^{n} \alpha_{i} \frac{\partial U_{0}}{\partial m_{i}}+\sum_{i=1}^{l} \beta_{i} \frac{\partial U_{0}}{\partial a_{i}}
$$

where the masses and spring parameters of the perturbed system are

$$
\begin{aligned}
\bar{m}_{i}=m_{i}+\varepsilon \alpha_{i}, & (i=1, \ldots, n) . \\
\bar{a}_{j}=a_{j}+\varepsilon \beta_{j}, & (j=1, \ldots, l) .
\end{aligned}
$$

While some of the $\alpha_{i}$ and $\beta_{j}$ may be zero, all are fixed, once chosen. 


\section{The Curved Modal Line}

Kuo [8] demonstrates

Theorem X-I: If the parent system

$$
\ddot{x}_{i}=\partial U_{0} / \partial x_{i}, \quad(i=1, \ldots, n)
$$

possesses, at some energy level $h$, a straight modal line, then the perturbed system

$$
\ddot{x}_{i}=\partial \sigma / \partial x_{i}, \quad(i=1, \ldots, n)
$$

moving at the same energy level $h$, possesses a unique modal line in an $\varepsilon$-neighborhood of that of the parent system.

The proof of this theorem is lengthy and will not be reproduced here.

In view of this theorem, the modal line of the perturbed system may be written as

$$
x_{i}\left(x_{1}\right)=c_{i} x_{1}+\varepsilon \xi_{1 i}\left(x_{1}\right)+\varepsilon^{2} \xi_{2 i}\left(x_{1}\right)+\ldots, \quad(i=2, \ldots, n)
$$

and this is valid everywhere except, possibly, on the $L_{a}$-surface where the system $M$ is singular.

Then, substituting (10.8) in (3.12); i.e., in the system $M$, and expanding in powers of $\varepsilon$, it turns out that $\xi_{1 i}$ must satisfy the system of linear, inhomogeneous, second-order equations

$$
\begin{gathered}
2\left(U_{0}^{*}+h\right) \xi_{1 i}^{\prime \prime}+\left[\frac{\partial U_{0}^{*}}{\partial x_{1}} \sum_{k=1}^{n} c_{k}^{2}\right] \xi_{1 i}^{\prime}+\left[\sum_{j=1}^{n}\left(c_{i} \frac{\partial^{2} U_{0}^{*}}{\partial x_{1} \partial x_{j}}-\frac{\partial^{2} U_{0}^{*}}{\partial x_{i} \partial x_{i}}\right) \sum_{k=1}^{n} c_{k}^{2}\right] \xi_{1 i} \\
=\left(\frac{\partial \omega_{0}^{*}}{\partial x_{i}}-c_{i} \frac{\partial \omega_{0}^{*}}{\partial x_{1}}\right) \sum_{k=1}^{n} c_{k}^{2} ; \quad(i=2, \ldots, n)
\end{gathered}
$$

where

$$
U_{0}^{*}=U_{0}\left(x_{1}, x_{2}^{*}\left(x_{1}\right), \ldots, x_{n}^{*}\left(x_{1}\right)\right)
$$

and a similar notation is used for $\omega_{0}^{*}$ and for the partial derivatives of these quantities. Moreover, it turns out that $\xi_{2 i}$ must satisfy a system of equations in which the left-hand side is identical to (10.9) while the righthand side becomes a function the $\xi_{1 i}$. Consequently, one can find $\xi_{2 i}$ if one can solve (10.9), and we need concern ourselves only with $(10.8)$; i.e., with first-order perturbations. 


\section{Integrable Cases}

There exist certain cases in which the solutions of $(\mathbf{1 0 . 9 )}$ is reducible to quadratures $[8,18]$. Where this cannot be done, $(10.9)$ must be integrated by numerical methods.

One of the integrable cases is that of weak coupling where the forces, exerted by the coupling springs under finite displacements, are of order $\varepsilon$; i.e.,

$$
\frac{\partial^{2} U_{0}^{*}}{\partial x_{i} \partial x_{j}}=O(\varepsilon), \quad(i \neq j ; \quad i, j=1, \ldots, n)
$$

Under the assumption of weak coupling, the system (10.9) reduces to

$$
\begin{gathered}
2\left(U_{0}^{*}+h\right) \xi_{i}^{\prime \prime}+\left(\frac{\partial U_{0}{ }^{*}}{\partial x_{1}} \sum_{k=1}^{n} c_{k}{ }^{2}\right) \xi_{i}{ }^{\prime}-\left(\frac{\partial^{2} U_{0}{ }^{*}}{\partial x_{i}{ }^{2}} \sum_{k=1}^{n} c_{k}{ }^{2}\right) \xi_{i} \\
=\left(\frac{\partial \omega_{0}{ }^{*}}{\partial x_{i}}-c_{i} \frac{\partial \omega_{0}{ }^{*}}{\partial x_{1}}\right) \sum_{k=1}^{n} c_{k}{ }^{2}, \quad(i=2, \ldots, n)
\end{gathered}
$$

where the subscript 1 on the $\xi_{i 1}$ has been deleted.

Defining the function

$$
U_{0}^{*}+h=G\left(x_{1}\right),
$$

it can be shown that the system (10.12) becomes the self-adjoint system

$$
2\left(G \xi_{i}^{\prime}\right)^{\prime}-\left(G^{\prime} \xi_{i}\right)^{\prime}=-\Omega_{i}\left(x_{1}\right), \quad(i=2, \ldots, n)
$$

where

$$
\Omega_{i}\left(x_{1}\right)=\sum_{k=1}^{n} c_{k}^{2} \int_{0}^{x_{1}}\left(c_{i} \frac{\partial \omega_{0}^{*}}{\partial x_{1}}-\frac{\partial \omega_{0}^{*}}{\partial x_{i}}\right) d x_{1}, \quad(i=2, \ldots, n) .
$$

The general solution of $(10.14)$ is

$$
\xi_{i}=\frac{1}{2} \sqrt{G\left(x_{1}\right)} \int_{0}^{x_{1}} \frac{A_{i}-\Omega_{i}(u)}{G(u)^{3 / 2}} d u+B_{i} \sqrt{\left(G\left(\overline{x_{1}}\right)\right.}
$$

where $A_{i}$ and $B_{i}$ are constants of integration.

Inasmuch as the modal line of the perturbed system must pass through the origin, one can readily show that $B_{i}=0,(i=2, \ldots, n)$. However, the only other condition on modal lines-that they intercept the $L_{a}$-surfacedoes not fix the values of the $A_{i}$. Nevertheless, it has been shown for systems 
with two degrees of freedom [8], and by Kuo [18] for those with any finite number of degrees of freedom, that the $A_{i}=0,(i=2, \ldots, n)$ as well, when coupling is weak. The arguments leading to this result are lengthy and not simple, even in systems with only two degrees of freedom, because the system of equations $M$ is singular on the $L_{a}$-surface. Therefore, we shall not reproduce them here. However, the result leads directly to

Theorem X-II: The slope at the origin of the modal line of the perturbed system is the same as that of its weakly coupled parent system, on which it neighbors.

It follows that the modal line of the perturbed system is given, to first order in $\varepsilon$, by

$$
x_{i}\left(x_{1}\right)=c_{i} x_{1}+\varepsilon \xi_{i}\left(x_{1}\right), \quad(i=2, \ldots, n)
$$

where

$$
\xi_{i}=-\frac{1}{2} \sqrt{G\left(x_{1}\right)} \int_{0}^{z_{1}} \frac{\Omega_{i}(u)}{G(u)^{3 / 2}} d u,
$$

and the $\Omega_{i}$ are defined in (10.15).

It is evident that the modal line of the perturbed system is curved in the case considered here because the modal line of the perturbed system has, at the origin, the same slope as has the straight modal line of the parent system, but everywhere else, these modal lines do not coincide. Hence, the corresponding normal-mode vibration is nonsimilar.

A number of examples, involving perturbations of mass and/or spring parameters have been computed $[8,18]$. In one of these [8], results of first-order perturbation theory have been compared with the actual behavior of the physical system. Even though, in that particular case, $|\varepsilon|$ is much larger than contemplated in perturbation theory, the agreement is excellent.

\section{General Properties}

It was observed in Theorem V-VI that every modal line, straight or curved, intercepts the $L_{a}$-surface orthogonally. One can also show readily by means of (3.12) that a curved modal line intersects no equipotential surface orthogonally, other than the $L_{a}$-surface. Moreover, Theorem V-V states that straight modal lines intersect every equipotential surface orthogonally. From these observations it follows that a straight modal line is invariant under changes of energy level $h$, but a curved modal line is not. In other words, if the modal line in a given mode of motion is curved, a new modal line must be computed for each value of $h$. 
Consider now (4.4) with $f_{1}(t) \equiv 0$; i.e., the equation determining the period of a normal-mode vibration. It is well known that the period $T$, given in (4.9), is independent of $X_{1}$ if (4.4) is linear, but it does depend on $X_{1}$ if (4.4) is nonlinear. Summarizing these results leads to an interesting gradation of normal-mode vibrations. In it, we replace the words "modal line" by "mode."

(i) In linear systems, the normal-mode vibrations are always similar; the normal-mode coordinates decouple the equations of motion for arbitrary motions; mode and period of the normal-mode vibration are independent of the energy level of the motion. Linear combinations of solutions are also solutions.

(ii) If the system is nonlinear, the normal-mode coordinates decouple the equations of motion for that mode only. If the normal-mode vibration is similar, the mode is independent of the energy level, but the period is not. Linear combinations of solutions are no longer solutions.

(iii) If the system is nonlinear and the normal-mode vibration is nonsimilar, mode as well as period depend on the energy level; otherwise, the conclusions are the same as in (ii) above.

\section{Exact Solutions to Steady-State Forced Vibrations [35]}

\section{Introductory Remarks}

Steady-state forced vibrations can only arise in nonautonomous systems. They are periodic motions of certain physical systems that are acted upon by time-dependent, equiperiodic forces. We employ

Definition III: Steady-state forced vibrations of an admissible, nonautonomous system are vibrations-in-unison having as their least period that of the exciting forces.

In all that follows, we shall assume (as is usually done in the linear problem) that only one of the masses, say $m_{1}$, is subjected to an admissible forcing function. Hence, the system $H$ is

$$
\begin{aligned}
& \ddot{x}_{1}=U_{x_{1}}\left(x_{1}, \ldots, x_{n}\right)+f_{1}(t), \\
& \ddot{x}_{i}=U_{x_{i}}\left(x_{1}, \ldots, x_{n}\right), \quad(i=2, \ldots, n),
\end{aligned}
$$

and the system $M$ is

$$
\begin{aligned}
& 2\left[U\left(x_{1}, \ldots, x_{n}\right)+h+F_{1}\left(x_{1}, X_{1}\right)\right] x_{i}{ }^{\prime \prime} \\
& \quad+\sum_{k=1}^{n} x_{k}{ }^{\prime 2}\left\{x_{i}{ }^{\prime}\left[U_{x_{1}}+f_{1}\left(t\left(x_{1}\right)\right)\right]-U_{x_{j}}\right\}=0, \quad(j=2, \ldots, n)
\end{aligned}
$$

where primes denote differentiation with respect to $x_{1}$. 
Among the systems represented by (11.1) and (11.2) there exists an entire class whose steady-state forced vibrations can be determined without approximation. It is clear that the linear system, for instance, belongs to that class. We shall show that exact solutions of the steady-state forced vibration problem can be found for the entire class of homogeneous systems under suitable excitation; among them is the linear system under simple harmonic excitation.

The technically most interesting information regarding steady-state forced vibrations is contained in the so-called "resonance curves" well known from linear theory. These are the families of curves in the frequencyamplitude planes which relate, for prescribed amplitudes of the forcing functions, the amplitudes $X_{i}$ of the motions in the various degrees of freedom to the frequency $\omega$ of the exciting force. In the steady-state vibrations of nonlinear systems these response curves $X_{i}(\omega),(i=1, \ldots, n)$ play a similar role. We shall show that these curves are integrals of equations of the form [35]

$$
\frac{d X_{i}}{d \omega}=\frac{P_{i}\left(X_{i}, \omega\right)}{Q_{i}\left(X_{i}, \omega\right)}, \quad(i=1, \ldots, n),
$$

and that the $X_{i} \omega$-planes contain certain singular points. The number, location, and character of the singular points of (11.3) can then be used to determine the geometrical character of the response curves. In particular, one can ascertain where (if at all) resonance occurs, and at what frequencies (if at all) the phenomenon of "tuned dynamic vibration absorption" takes place.

\section{Homogeneous Systems}

The steady-state response of the linear system to periodic excitation is normally discussed for the case when the excitation is simple harmonic $[36,37]$. In fact, the response curves cease to have meaning under general periodic excitation. Since the steady-state response of the linear system to simple harmonic excitation is, again, simple harmonic, it turns out that in that case,

$$
x_{i}(t) / f(t) \equiv \text { const., } \quad(i=1, \ldots, n) .
$$

We shall show that the linear system under simple harmonic excitation is merely a special case of the homogeneous system under the excitation $\left[\mathcal{F}_{1}(t)\right]^{k}$ where

$$
f_{1}(t)=P_{0} \operatorname{cam}(n \Omega t),
$$


and the steady-state response of this class is of the form

$$
x_{i}(t)=X_{i} \operatorname{cam}(n \Omega t), \quad(i=1, \ldots, n) ;
$$

hence, (11.5) and (11.6) are such that (11.4) is satisfied for all $k$ in $1 \leqslant k<\infty$ (or $1 \leqslant n<\infty$ ). We shall make this demonstration only for a system having two degrees of freedom [35], but we indicate the results of generalizing to any number of degrees of freedom [12].

Let the degrees of freedom be

$$
u_{1}=u, \quad u_{2}=v
$$

and write the forcing function in the form

$$
f_{1}(t)=P_{0}^{1 / k} \operatorname{cam}\left(n \sqrt{\frac{D \lambda^{2}}{n}} t\right)
$$

where

$$
D=(c X)^{k-1},
$$

and $c$ is a constant. Form (11.8) has a certain advantage over (11.5). In (11.8), the quantity $\lambda$ plays a role similar to that of the circular frequency of simple harmonic excitation, but it is not the frequency of excitation when $k>1$. Hence, $\lambda$ will be called the generalized circular frequency.

Then, the equations of motion of the physical system are

$$
\begin{aligned}
& m_{1} \ddot{u}+a_{1} u^{k}+a_{2}(u-v)^{k}=P_{0} \operatorname{cam}^{k}\left(n \sqrt{\left(D \lambda^{2} / n\right)} t\right), \\
& m_{2} \ddot{v}+a_{3} v^{k}-a_{2}(u-v)^{k}=0,
\end{aligned}
$$

those of the pseudo-system are

$$
\ddot{x}+\frac{a_{1}}{\sqrt{m_{1}}}\left(\frac{x}{\sqrt{m_{1}}}\right)^{k}+\frac{a_{2}}{\sqrt{m_{1}}}\left(\frac{x}{\sqrt{m_{1}}}-\frac{y}{\sqrt{m_{2}}}\right)^{k}=\frac{P_{0}}{\sqrt{m_{1}}} \operatorname{cam}^{k}\left(n \sqrt{\frac{D \lambda^{2}}{n}} t\right),
$$

$$
\ddot{y}+\frac{a_{3}}{\sqrt{m_{2}}}\left(\frac{y}{\sqrt{m_{2}}}\right)^{k}-\frac{a_{2}}{\sqrt{m_{2}}}\left(\frac{x}{\sqrt{m_{1}}}-\frac{y}{\sqrt{m_{2}}}\right)^{k}=0,
$$

and the equation of the trajectories is

$$
\begin{aligned}
2\{- & \frac{1}{k+1}\left[a_{1}\left(\frac{x}{\sqrt{m_{1}}}\right)^{k+1}+a_{3}\left(\frac{y}{\sqrt{m_{2}}}\right)^{k+1}+a_{2}\left(\frac{x}{\sqrt{m_{1}}}-\frac{y}{\sqrt{m_{2}}}\right)^{k+1}\right] \\
& \left.+\int_{x}^{x} \frac{P_{0}}{\sqrt{m_{1}}} \operatorname{cam}^{k}\left(n \sqrt{\frac{D \lambda^{2}}{n}} t(x)\right) d x+h\right\} y^{\prime \prime}+\left(1+y^{\prime 2}\right)\left\{y ^ { \prime } \left[-\frac{a_{1}}{\sqrt{m_{1}}}\left(\frac{x}{\sqrt{m_{1}}}\right)^{k}\right.\right.
\end{aligned}
$$




$$
\begin{aligned}
& \left.\left.+\frac{a_{2}}{\sqrt{m_{1}}}\left(\frac{x}{\sqrt{m_{1}}}-\frac{y}{\sqrt{m_{2}}}\right)^{k}+\frac{P_{0}}{\sqrt{m_{1}}} \operatorname{cam}^{k}(n] \sqrt{\frac{D \lambda^{2}}{n}} t(x)\right)\right] \\
& \left.-\left[\frac{a_{3}}{\sqrt{m_{2}}}\left(\frac{y}{\sqrt{m_{2}}}\right)^{k}+\frac{a_{2}}{\sqrt{m_{2}}}\left(\frac{x}{\sqrt{m_{1}}}-\frac{y}{\sqrt{m_{2}}}\right)^{k}\right]\right\}=0 .
\end{aligned}
$$

Simple substitution of

$$
x(t)=X \operatorname{cam}\left(n \sqrt{\frac{\overline{D \lambda^{2}}}{n} t}\right),
$$

$$
y=c x
$$

into (11.11) and (11.12) (and making use of the formulas (9.33) for the derivatives of cam-functions) shows that (11.13) are indeed the solutions, and the constant $c$ in the second of (11.13) is the same as that in (11.9).

But it follows from (11.13) that, in the case considered, $x(t)$ and $y(t)$ are cam-functions of the same argument. Hence, the steady-state vibrations are similar, or the trajectories satisfying (11.12) are straight lines.

If we define

$$
Y=c X
$$

the equations (11.11) and (11.12), with (11.13) and (11.14) substituted in them, are three equations in the three unknowns $X, Y$ and $c$, and they can be solved explicitly for these quantities.

One finds

$$
\begin{aligned}
c & =a_{2}^{1 / k} /\left\{a_{2}{ }^{1 / k}+\left(a_{3}-m_{2}^{(k+1) / 2} \lambda^{2}\right)^{1 / k}\right\}, \\
X^{k} & =\left\{P_{0} m_{1}^{k / 2}\left[a_{2}^{1 / k}+\left(a_{3}-m_{2}^{(k+1) / 2} \lambda^{2}\right)^{1 / k}\right]^{k}\right\} / H, \\
Y^{k} & =\left\{P_{0} a_{2} m_{2}^{k / 2}\right\} / H
\end{aligned}
$$

where

$$
\begin{aligned}
H= & a_{1}\left[a_{2}^{1 / k}+\left(a_{3}-m_{2}^{(k+1) / 2} \lambda^{2}\right)^{1 / k}\right]^{k}+a_{2}\left(a_{2}+a_{3}-m_{2}^{(k+1) / 2} \lambda^{2}\right) \\
& -\left(m_{2} / m_{1}\right)^{(k-1) / 2} m_{1}^{(k+1) / 2} a_{2}^{(k-1) / k}\left[a_{2}^{1 / k}+\left(a_{3}-m_{2}^{(k+1) / 2} \lambda^{2}\right)^{1 / k}\right]-a_{2}^{2} .
\end{aligned}
$$

If the system has more than two degrees of freedom, say $m$, the equations of motion of the pseudo-system are 


$$
\begin{aligned}
& \left.\ddot{x}_{1}=-\frac{a_{01}}{\sqrt{m_{1}}}\left(\frac{x_{1}}{\sqrt{m_{1}}}\right)^{k}-\sum_{j=2}^{n} \frac{a_{1 j}}{\sqrt{m_{1}}}\left(\frac{x_{1}}{\sqrt{m_{1}}}-\frac{x_{j}}{\sqrt{m_{j}}}\right)^{k}+\frac{P_{0}}{\sqrt{m_{1}}} \operatorname{cam}^{k}(n] \sqrt{\frac{D \lambda^{2}}{n}} t\right), \\
& \ddot{x}_{j}=-\frac{a_{j 0}}{\sqrt{m_{j}}}\left(\frac{x_{j}}{\sqrt{m_{j}}}\right)^{k}-\sum_{l=1}^{m} \frac{a_{j l}}{\sqrt{m_{j}}}\left(\frac{x_{j}}{\sqrt{m_{j}}}-\frac{x_{l}}{\sqrt{m_{l}}}\right)^{k}, \quad(j=2, \ldots, m),
\end{aligned}
$$

and substitution shows that

$$
\begin{gathered}
x_{1}=X_{1} \operatorname{cam}\left(n \sqrt{\frac{D \lambda^{2}}{n} t}\right) \\
x_{j}(t) / x_{1}(t) \equiv c_{j}=X_{j} / X_{1}, \quad(j=2, \ldots, m)
\end{gathered}
$$

satisfy this system as well as the equations of the trajectory which are not reproduced here. Now, there are $m$ quantities $X_{j}$ and $m-1$ quantities $c_{i}$ which constitute the unknowns, and there are $m$ equations of motion and $m-1$ equations of the trajectory. Hence, the problem is determinate [12].

It is now advantageous, as done in the linear forced vibration problem, to introduce non-dimensional quantities as follows:

$$
\begin{aligned}
\sqrt{m_{1}} \bar{x} & =x /\left(P_{0} / a_{1}\right)^{k}, \quad \sqrt{m_{1}} \bar{X}=X /\left(P_{0} / a_{1}\right)^{k}, \\
\sqrt{m_{2}} \bar{y} & =y /\left(P_{0} / a_{1}\right)^{k}, \quad \sqrt{m_{2}} \bar{Y}=Y /\left(P_{0} / a_{1}\right)^{k}, \\
\mu_{i j}^{2} & =m_{i} / m_{j}, \quad \alpha_{i j}=a_{i} / a_{j}, \quad \mu_{21} \bar{c}=c, \\
\gamma & =\lambda^{2} /\left[a_{2} / m_{2}^{(k+1) / 2}\right]
\end{aligned}
$$

where it should be remembered that $\bar{X}$ and $\bar{Y}$ are nondimensional amplitudes, and $\gamma$ is a nondimensional generalized frequency ratio. With the introduction of these quantities, (11.15) become

$$
\begin{aligned}
\bar{c} & =[G(\gamma)]^{-1}, \\
\bar{X}^{k} & =[G(\gamma)]^{k} / H(\gamma), \\
\bar{Y}^{k} & =[H(\gamma)]^{-1}
\end{aligned}
$$

where

$$
\begin{aligned}
& G(\gamma)=1+\left(\alpha_{32}-\gamma\right)^{1 / k}, \\
& H(\gamma)=[G(\gamma)]^{k}+\alpha_{21}\left(1+\alpha_{32}-\gamma\right)-\alpha_{21} \gamma \mu_{12}^{2} G(\gamma)-\alpha_{21}
\end{aligned}
$$


One must now find the locus of the endpoints of the trajectories, i.e., the $L$-surface, which is, here, a curve. The $L$-curves are found as follows: the first of (11.20) is solved for $\gamma$ as a function of $\bar{c}$. This is substituted in the second, thus eliminating $\gamma$, and resulting in an equation of the form

$$
X^{k}=\theta(\bar{c}) .
$$

If one substitutes in that equation

$$
\bar{c}=\bar{Y} / \bar{X},
$$

one finds an equation in $\bar{X}$ and $\bar{Y}$; this is the equation of one of the loci of end-points of the trajectories. The second locus is found by substituting $-\bar{X}$ for $\bar{X}$; then, the equations of the two loci become [35]

$$
\bar{X}^{k} \bar{Y}+\alpha_{21}(\bar{X}-\bar{Y})^{k}\left(\bar{Y}+\mu_{12}^{2} \bar{X}\right)-\alpha_{21} \alpha_{32} \mu_{12}^{2} \bar{X} \bar{Y}^{k} \mp \bar{Y}=0 .
$$

From these equations one can readily deduce the following properties of the $L$-curves:

(i) they intersect the $\bar{y}$-axis at $\bar{y}=0$ and at $\bar{y}= \pm \alpha_{12}^{-1 / k}$;

(ii) they intersect the $\bar{x}$-axis at the origin only;

(iii) in the linear system $(k=1)$, they are hyperbolas;

(iv) as $|\bar{X}|$ and $|\bar{Y}| \rightarrow \infty, \bar{Y} \mid \bar{X} \rightarrow$ constants equal to the slope of the modal lines of the autonomous system $[\mathbf{3 5}]$.

The last property proves the following

Theorem XI-I: As the generalized frequency ratio $\gamma \rightarrow \gamma_{1,2}$, where $\gamma_{1,2}$ are the generalized frequency ratios of normal-mode vibrations of the autonomous system, the nondimensional amplitudes $|\bar{X}|,|\bar{Y}| \rightarrow \infty$. Hence, the system goes into resonance in the neighborhood of free normal-mode vibrations.

The $L$-curves and trajectories of steady-state vibrations have been computed for the nonlinear problem

$$
\mu_{12}^{2}=2, \quad \alpha_{21}=1 / 2, \quad \alpha_{32}=3, \quad k=3
$$

and for the associated linear problem in which all parameters have the values given in (11.25), except that $k=1$. The results in the $\bar{x} \bar{y}$-plane are shown for these two examples in Figures 10(a) and (b), respectively.

For an understanding of the general theory, to be presented in the next section, it is helpful to interpret these diagrams. In general, the trajectories of steady-state forced vibrations are, here, segments of straight lines 


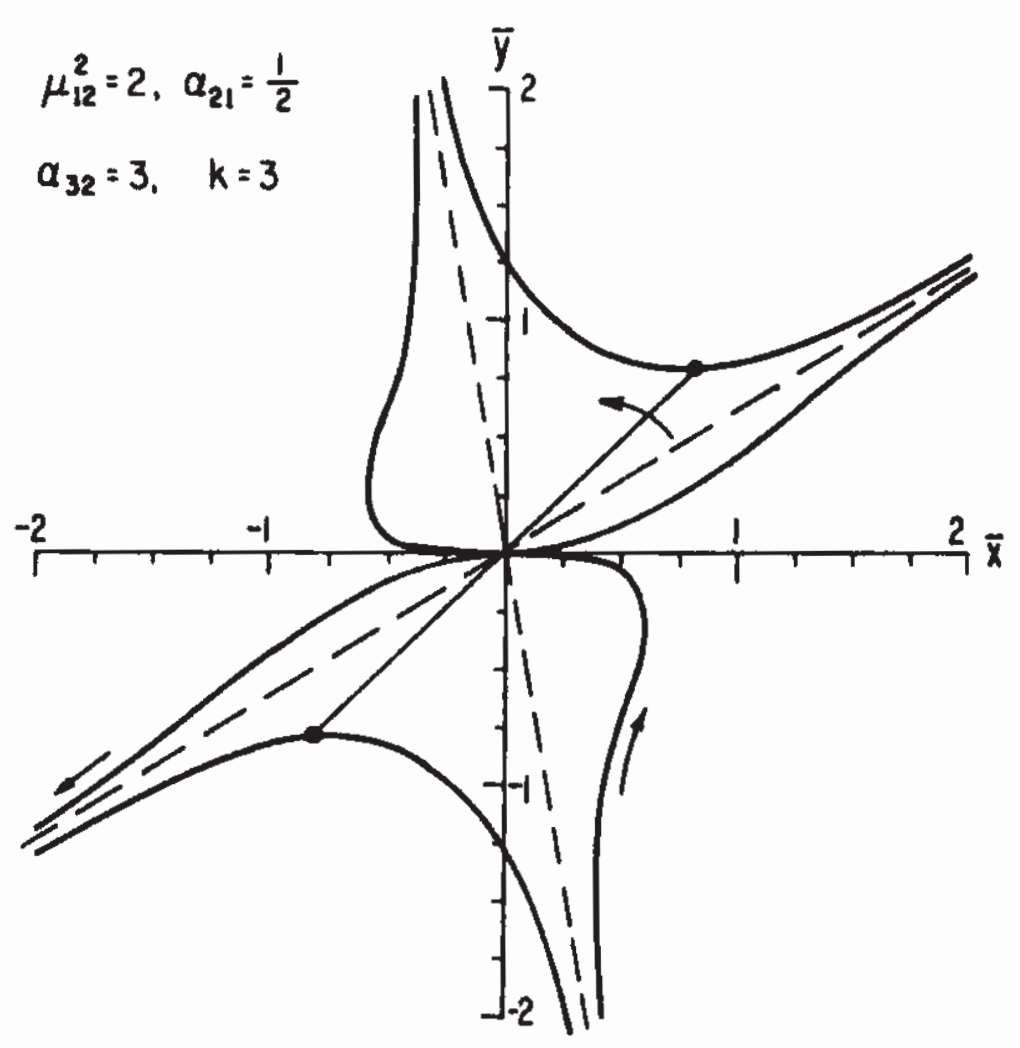

(a)

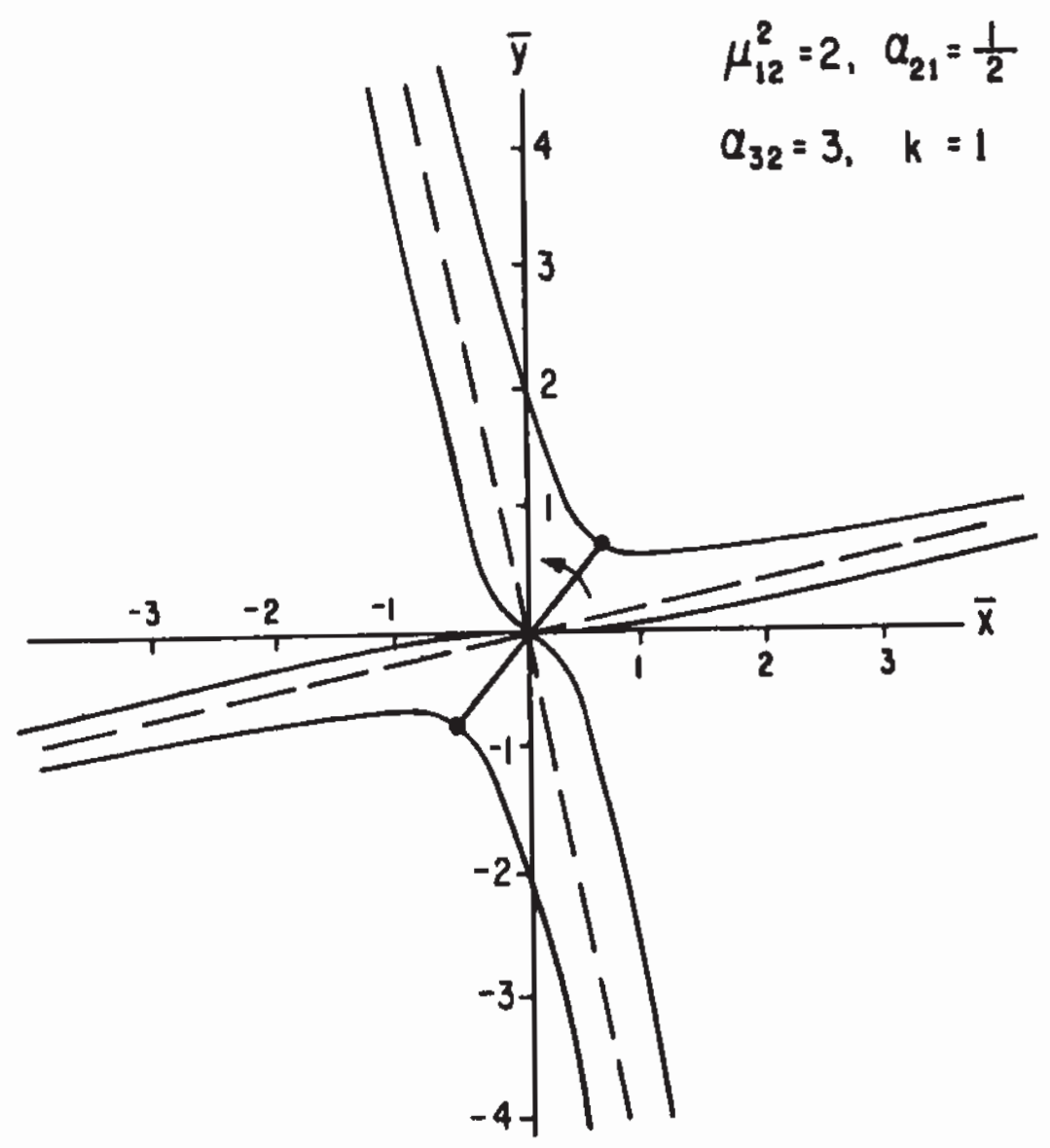

(b)

Fig. 10. Trajectory of steady-state forced vibration in (a) nonlinear and (b) corresponding linear systems. 
that pass through the origin and terminate on the $L$-curves. Their slope is a function of the frequency $\gamma$ and, in fact, their slope $\bar{c}(\gamma)$ is given in the first of (11.20).

Assume that $\gamma$, i.e., essentially the frequency of excitation, is small, so that $\bar{c}$ is less than the slope of the modal line in the first and third quadrant (shown dotted). As $\gamma$ is increased, the trajectory begins to rotate in a counterclockwise direction and becomes longer, tending to infinity as the slope $\bar{c}(\gamma)$ approaches that of the modal line. As $\gamma$ is further increased, the trajectory continues to rotate, but it becomes now shorter until $\bar{c}=\infty$. This occurs where $G(\gamma)=0$ and, at this frequency, there is bounded $\bar{y}$-motion, but no $\bar{x}$-motion. In other words, the $\bar{x}$-motion vanishes identically because $m_{2}$ acts as a tuned dynamic vibration absorber for $m_{1}$. A further increase in $\gamma$ will further rotate the trajectory, and the motion becomes out-of-phase because the slope $\bar{c}<0$. At first, the trajectory will lengthen until the motion becomes, again, unbounded when $\gamma=\gamma_{2}$. However, further increases in $\gamma$ will reduce the amplitudes of the steady-state forced response, and these amplitudes tend to zero as $\gamma \rightarrow \infty$.

\section{The Response Curves [35]}

The differential equations, satisfied by the response curves, are found from the second and third of (11.20); they are

$$
\begin{aligned}
& \frac{d \bar{X}}{d \gamma}=\frac{\bar{X} F(\gamma)}{k G(\gamma) H(\gamma)}, \\
& \frac{d \bar{Y}}{d \gamma}=-\frac{\bar{Y} H^{\prime}(\gamma)}{k H(\gamma)}
\end{aligned}
$$

where

$$
F(\gamma)=k G^{\prime}(\gamma) H(\gamma)-G(\gamma) H^{\prime}(\gamma),
$$

and $G$ and $H$ are defined in (11.21).

Both of (11.26) are seen to have singular points on the $\gamma$-axes at the zeros of $H(\gamma)$. The first of them has additional singular points on the $\gamma$-axis at the zeros of $G(\gamma)$.

Now, the generalized frequency ratios of normal-mode vibrations of the autonomous system are, in general, functions of the amplitudes $\bar{X}$ and $\bar{Y}$, and they tend to certain limits as the amplitudes tend to zero. It can be shown $[12,13]$ that their limiting values coincide with the zeros of $H(\gamma)$. In other words, the equation

$$
H(\gamma)=0
$$


can be shown to play the part of the so-called "frequency equation" of the autonomous system. Hence, both equations (11.26) have singular points at the natural frequencies of normal-mode vibrations at vanishingly small amplitudes.

Denote the zeros of $H(\gamma)$ by $\gamma_{n}$. Then expanding the first of (11.26) in the neighborhood of $\left(\bar{X}=0, \gamma=\gamma_{n}\right)$ one finds

$$
\frac{d \bar{X}}{d \gamma_{n}}=-\frac{\bar{X}}{\gamma_{n} k}+\ldots
$$

because

$$
F\left(\gamma_{n}\right)=-G\left(\gamma_{n}\right) H^{\prime}\left(\gamma_{n}\right)
$$

Expanding the second of (11.26), one has directly

$$
\frac{d \bar{Y}}{d \gamma_{n}}=-\frac{\bar{Y}}{\gamma_{n} k}+\ldots
$$

The integrals of (11.29) and (11.31) are

$$
\bar{X} \gamma_{n}{ }^{k}=\text { const., } \quad \bar{Y} \gamma_{n}{ }^{k}=\text { const. }
$$

These are equations of hyperbolas; hence, the singular points on the $\gamma$-axes at the zeros of $H(\gamma)$ are for both (11.26) saddles.

Let the zeros of $G(\gamma)$ be denoted by $\gamma_{m}$. Then, expanding the first of (11.26) in the neighborhoods of $\left(X=0, \gamma=\gamma_{m}\right)$ one finds

$$
\frac{d X}{d \gamma_{m}}=\frac{X}{\gamma_{m}}+\ldots
$$

because

$$
F\left(\gamma_{m}\right)=k G^{\prime}\left(\gamma_{m}\right) H\left(\gamma_{m}\right)
$$

The integrals of (11.33) are the straight lines

$$
\bar{X} / \gamma_{m}=\text { const. }
$$

Hence, the singular points of the first of (11.26) on the $\gamma$-axis at the zeros of $G(\gamma)$ are star points (nodes).

Now, it follows from the definition (11.21) that $G(\gamma)$ has only one real zero at

$$
\gamma_{m}=\gamma^{*}=1+\alpha_{32} .
$$

Hence, we have [35] 


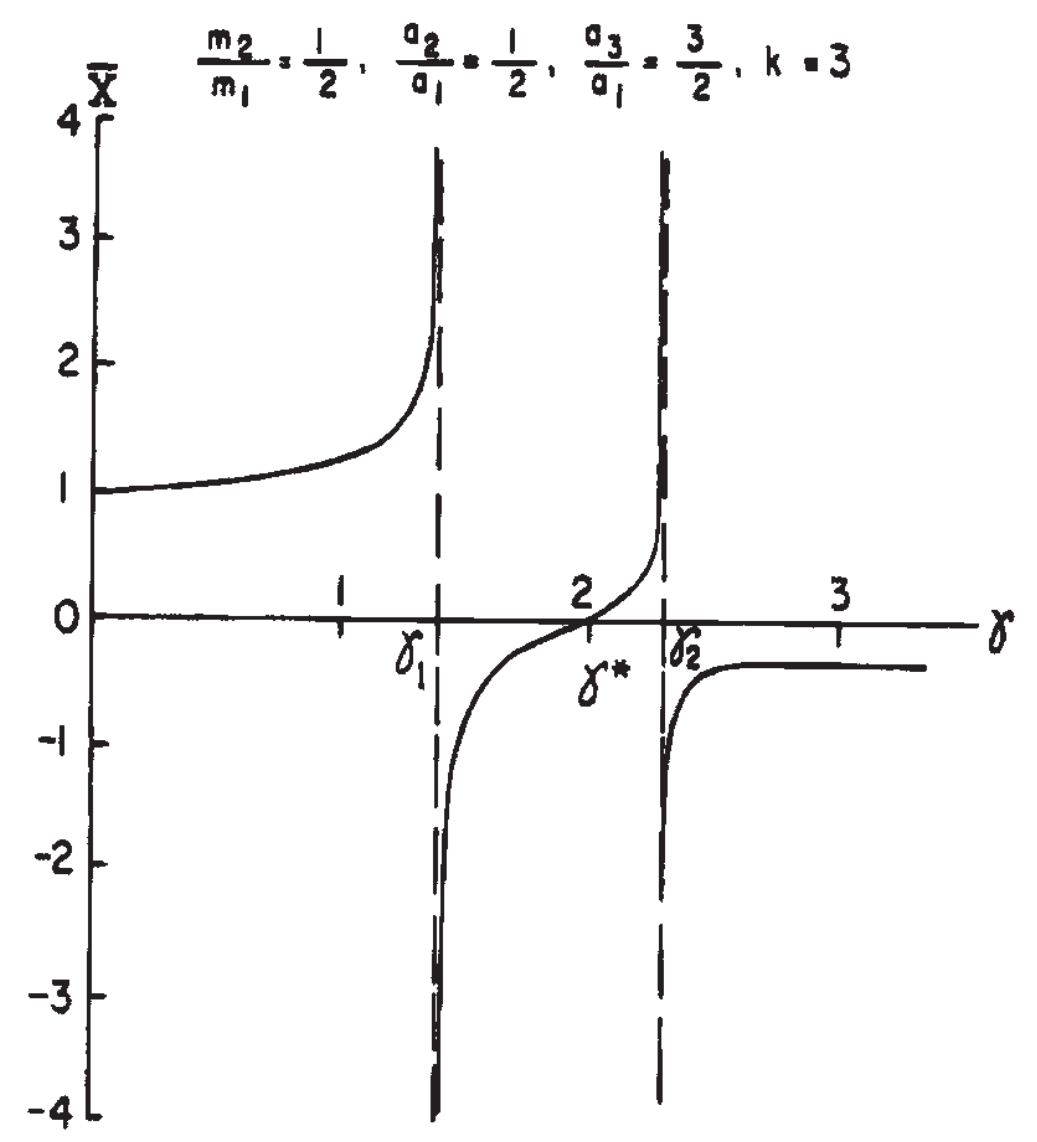

(a)

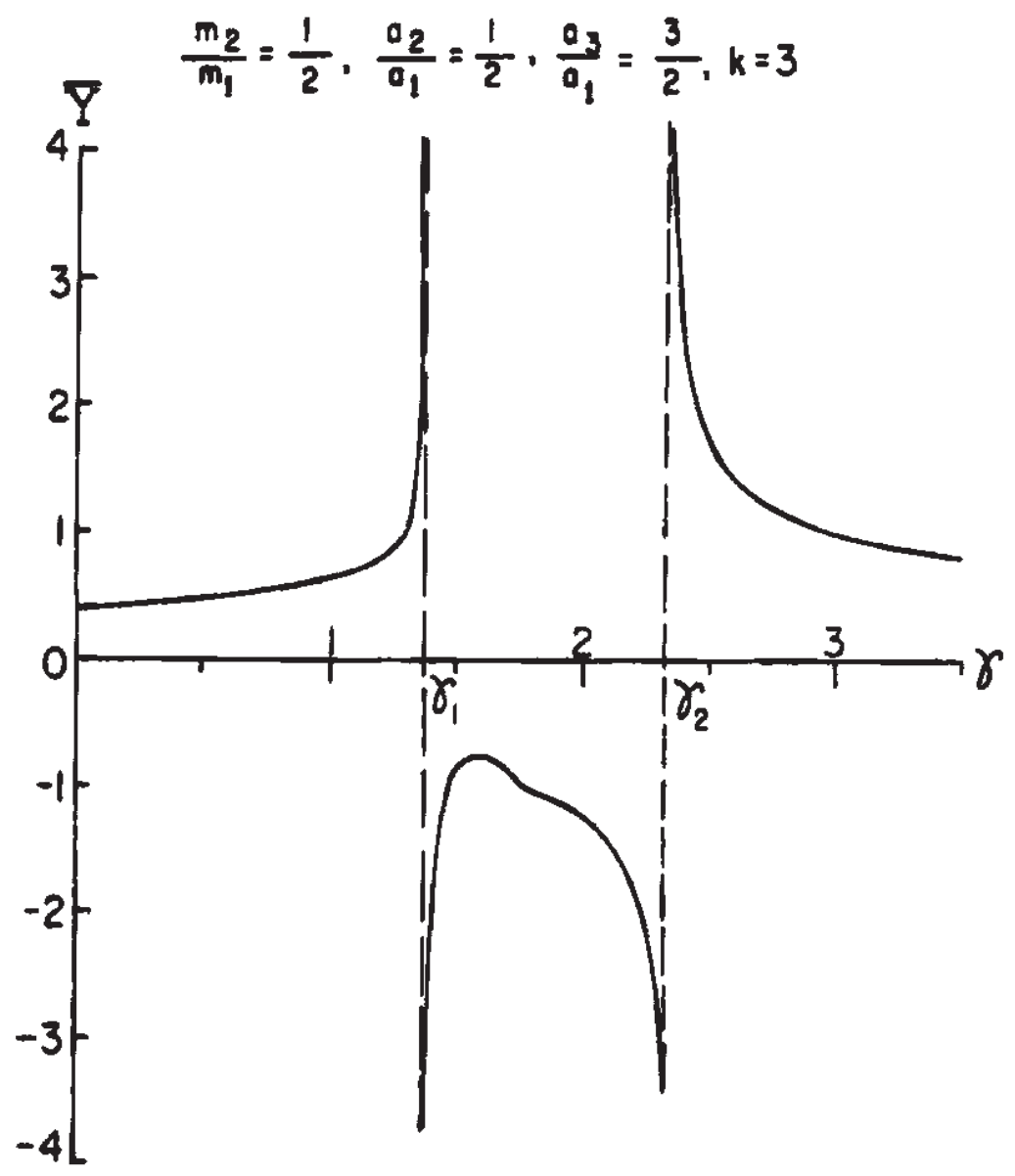

(b)

FrG. 11. Frequency-amplitude curves in (a) $\bar{X} \gamma$-plane and (b) $\bar{Y} \gamma$-plane. 


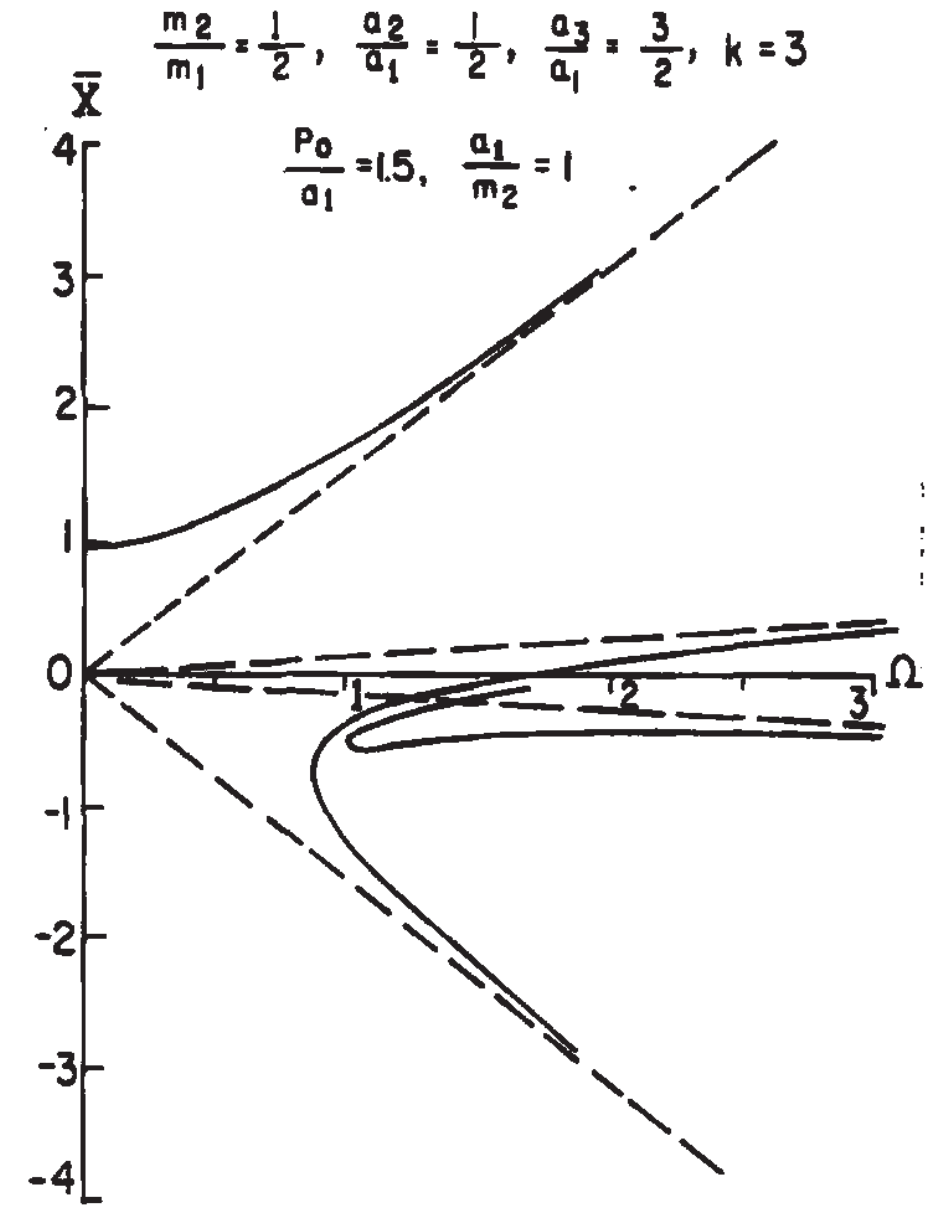

(a)

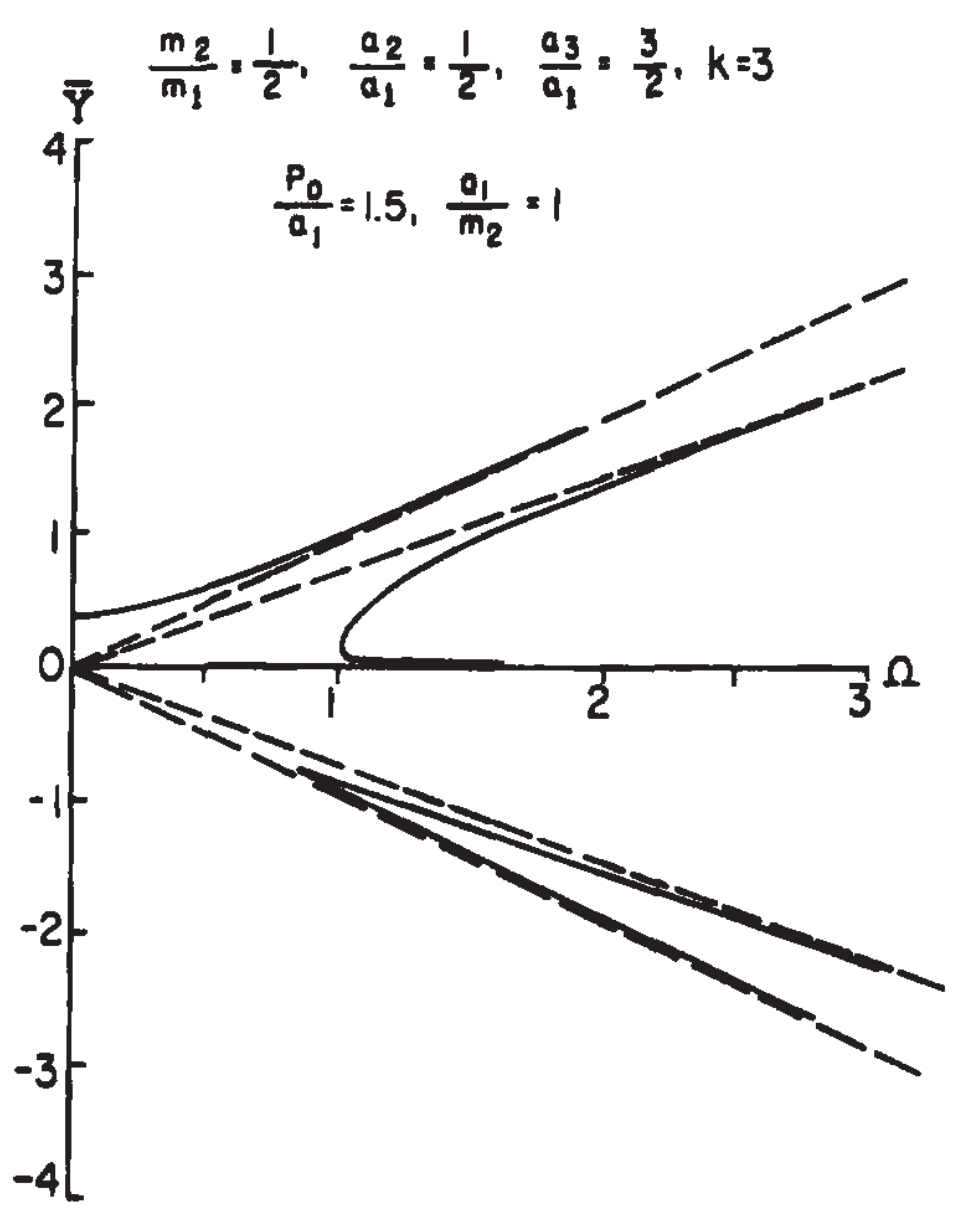

(b)

FIG. 12. Frequency-amplitude curves in physical $\bar{X} \Omega$-plane (a) and $\bar{Y} \Omega$-plane (b).

Theorem XI-II: The system (11.11) is such that, for any $k$ in $1 \leqslant k<\infty$, there exists a single nondimensional generalized frequency ratio $\gamma^{*}$ of the exciting force such that the mass $m_{2}$ acts as a tuned dynamic vibration absorber for the mass $m_{1}$ that is being excited.

Response curves in the $\bar{X} \gamma$ and $\bar{Y} \gamma$-planes for the values of the parameter given in (11.25) are shown in Figures $11(a)$ and (b), respectively.

It remains to map the $\bar{X} \gamma$ and $\bar{Y} \gamma$-planes on the $\bar{X} \Omega$ and $\bar{Y} \Omega$-planes, where the frequency $\Omega$ of the forcing function is defined through (11.5). It follows from (9.23) and (11.19) that this mapping is given by [35]

$$
\Omega=\pi \sqrt{n}\left\{\left[\mu_{12}^{2} \bar{c}(\gamma)\left(P_{0} / a_{1}\right)^{1 / k} X(\gamma)\right]^{k-1} \frac{a_{2}}{m_{2}} \gamma\right\}^{1 / 2}\left[B\left(\frac{1}{2 n}, \frac{1}{2}\right)\right]^{-1},
$$

and by a similar formula involving $\bar{Y}$ which is found by replacing $\bar{X}$ in (11.37) by $\bar{Y} / \bar{c}$. The results of this mapping for the example of Figure 11 is shown in Figures 12(a) and (b). 
XII. Steady-State Forced Vibrations by Approximate Methods

\section{Description of the Method [12]}

The problems of steady-state forced vibrations, considered here, are those of systems that neighbor on systems of known vibrations-in-unison. Two cases may arise: either the vibrations-in-unison of the unperturbed (parent) system are themselves steady-state forced vibrations of the parent system, or the vibrations-in-unison of the parent system are normal-mode vibrations of that system. It is clear that in the first case the amplitude of the forcing function need not be small, but in the second case, that amplitude must be small in absolute value because the nonautonomous system now neighbors on the autonomous one. In either of these two cases, the problem of finding steady-state forced vibrations can be reduced to the perturbation problem discussed in the section on nonsimilar normal-mode vibrations. The results reported here are, largely, due to Kinney [12].

We consider a parent system of potential function

$$
U=U\left(x_{1}, \ldots, x_{n}\right)
$$

for which at least one vibration-in-unison is known. Let the trajectory in configuration space of this vibration-in-unison be given by the (known) functions

$$
x_{i}=x_{i}^{*}\left(x_{1}\right), \quad(j=2, \ldots, n) .
$$

Now, the only vibrations-in-unison that have been deduced are either straight lines, or they are curved in the sense treated in the section on nonsimilar vibrations. Hence, they are always given by

$$
x_{j}^{*}\left(x_{1}\right)=c_{j} x_{1}+O\left(\varepsilon_{0}\right)
$$

where $\left|\varepsilon_{0}\right|$ is small, and when the trajectory is straight, the terms of order $\varepsilon_{0}$ are absent in (12.3).

The time-history of $x_{1}$ of the known vibration-in-unison is the (known) function

$$
x_{1}=x_{1}^{*}(t),
$$

and the time-histories of the other $x_{i}$ are found by substituting (12.4) in (12.3). Since the motion is a vibration-in-unison, (12.4) possesses on the interval $\left[0, X_{1}\right]$ (where $X_{1}$ is defined by $\dot{x}_{1}\left(X_{1}\right)=0$ ) the inverse

$$
t=t^{*}\left(x_{1}\right) \text {. }
$$

We shall, first, suppose that the parent system is nonautonomous, or the unit mass of the unperturbed pseudo-system is subjected to a force in the $f_{1}$-direction given by

$$
f_{1}=f(t)=f(t+T)
$$


where $T$ is the only period of $f$. Hence, the circular frequency of the periodic force is

$$
\omega=2 \pi / T
$$

We consider a neighboring, or perturbed system of potential function

$$
\tilde{U}=U\left(x_{1}, \ldots, x_{n}\right)+\varepsilon \psi\left(x_{1}, \ldots, x_{n}\right)
$$

where $\psi\left(x_{1}, \ldots, x_{n}\right)$ is a given perturbation potential which satisfies the admissibility conditions, and

$$
\varepsilon \psi=O(\varepsilon)
$$

everywhere where $\psi$ is defined. Then, the trajectories of the perturbed system are the $x_{j}\left(x_{1}\right),(j=2, \ldots, n)$ which satisfy

$$
2\left[U+\varepsilon \psi+F\left(x_{1}, X_{1}\right)+h\right] x_{i}^{\prime \prime}+\sum_{k=1}^{n} x_{k}{ }^{\prime 2}\left\{x_{j}{ }^{\prime}\left[U_{x_{1}}+\varepsilon \psi_{x_{1}}+f\left(\left(x_{1}\right)\right)\right]\right.
$$

$$
\left.-\left(U_{x_{j}}+\varepsilon \psi_{x_{j}}\right)\right\}=0, \quad(j=2, \ldots, n),
$$

and this equation is regular everywhere. Hence, one may construct the solutions

$$
x_{j}\left(x_{1}\right)=x_{j}\left(x_{1}, \varepsilon\right)=c_{j} x_{1}+\varepsilon \xi_{j}\left(x_{1}\right)+\ldots
$$

In a similar way,

$$
\begin{gathered}
t\left(x_{1}\right)=t\left(x_{1}, \varepsilon\right)=t_{0}\left(x_{1}\right)+\varepsilon t_{1}\left(x_{1}\right)+\ldots \\
t\left(x_{1}, 0\right)=t_{0}\left(x_{1}\right)=t^{*}\left(x_{1}\right)
\end{gathered}
$$

i.e., $t^{*}\left(x_{1}\right)$ is the zeroth order approximation of $t\left(x_{1}\right)$.

If one now uses an iteration scheme with respect to $t\left(x_{1}\right)$, combined with conventional perturbation techniques for the $x_{j}\left(x_{1}\right)$, the problem of determining the steady-state forced vibrations reduces to that of (10.9), treated in the section on nonsimilar normal-mode vibrations. The procedure consists in replacing $t\left(x_{1}\right)$ by $t_{0}\left(x_{1}\right)$ in (12.10) and then using conventional perturbation methods; these result in a linear nonhomogeneous system of equations which the $\xi_{j}\left(x_{1}\right)$ must satisfy. Now, the solutions of that system are functions of $t_{0}\left(x_{1}\right)$, and this will be indicated by using an appropriate superscript on the $\xi_{j}$. Using the notation

$$
Q^{*}\left(x_{1}\right)=Q\left(x_{1}, c_{2} x_{1}, \ldots, c_{n} x_{1}\right)
$$

for $U$ and $\psi$ and their partial derivatives, and using the definition of $F\left(x_{1}, X_{1}\right)$ given in (3.10), the equations of the $\xi_{j}$ turn out to be 


$$
\begin{aligned}
& 2\left[U^{*}+\int_{X_{1}}^{x_{1}} f\left(t_{0}\left(x_{1}\right)\right) d x_{1}+h\right] \xi_{j}^{(0)^{\prime \prime}}+\sum_{k=1}^{n} c_{k}{ }^{2}\left\{\left[U_{x_{1}}^{*}+f\left(t_{0}\left(x_{1}\right)\right)\right] \xi_{j}(0)^{\prime}\right. \\
& \left.+\sum_{l=2}^{n}\left[\left(c_{j} U_{x_{1} x_{l}}^{*}-U_{x_{j} x_{l}}^{*}\right) \xi_{l}^{(0)}\right]\right\}=\sum_{k=1}^{n} c_{k}^{2}\left(\psi_{x_{j}}^{*}-c_{j} \psi_{x_{1}}^{*}\right), \quad(j=2, \ldots, n) .
\end{aligned}
$$

But, this system of equations is of the form (10.9), and questions of its integration are discussed in the section on nonsimilar normal-mode vibrations, and also by Kinney [12] for different examples.

Once the integrals $\xi_{j}{ }^{(0)}\left(x_{1}\right)$ have been found the functions (12.11)

$$
x_{j}\left(x_{1}\right)=c_{j} x_{1}+\varepsilon \xi_{j}{ }^{(0)}\left(x_{1}\right), \quad(j=2, \ldots, n)
$$

are substituted in the first of the equations of motion; that equation can then be integrated by quadratures for the conditions

$$
\begin{array}{lll}
\dot{x}_{1}=0 & \text { when } & x_{1}=X_{1}, \\
x_{1}=X_{1} & \text { when } & t=0 .
\end{array}
$$

This results in a function

$$
x_{1}=x_{1}\left(t, X_{1}\right)
$$

and its inverse

$$
t=t_{1}\left(x_{1}\right)
$$

This latter is the first approximation of $t\left(x_{1}\right)$ (as compared to the zeroth approximation), and it may now be substituted in (12.10), and the entire procedure is repeated. Kinney [12] shows that it is more convenient to introduce the transformation

$$
\tau=\omega t
$$

and he has computed a number of interesting examples, including that of the tuned dynamic vibration absorber of weakly nonlinear two-degree-offreedom systems.

If the parent system is autonomous, one simply deletes the terms in (12.10) involving $f\left(t\left(x_{1}\right)\right)$ and $F\left(x_{1}, X_{1}\right)$, and one replaces $\psi\left(x_{1}, \ldots, x_{n}\right)$ by $g\left(t\left(x_{1}\right)\right)$ where $g(t)$ is periodic of period $T$. In view of (12.9), this latter step implies that the forcing function has small amplitude, and the result will be that of steady-state forced vibrations in the neighborhood of the vibrationsin-unison of the autonomous nonlinear system. It may be interesting to interpret the results of forced steady-state vibrations neighboring on the 
vibrations-in-unison of a parent system. This interpretation will be made in terms of trajectories in the configuration space, and of response curves in the frequency-amplitude planes. As an example, we consider a parent system that is linearizable within the meaning of $(2.11)$.

Consider the $n$-space and an equipotential surface indicated in Figure 13 together with the modal lines; in this diagram, the modal lines are surrounded by $\varepsilon$-tubes. Then, the desired trajectories of the steady-state

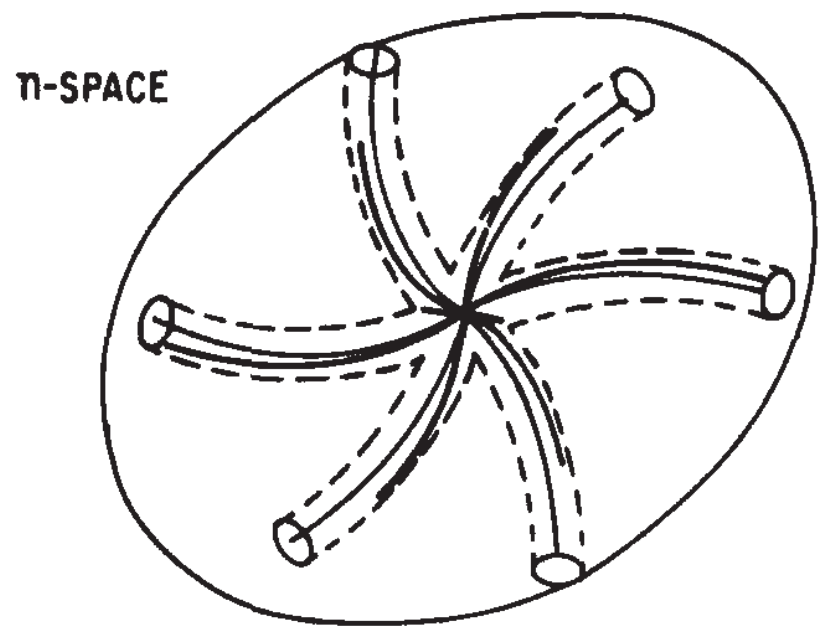

FIg. 13. Trajectories of free and forced vibrations in nonlinear $n$-degree-of-freedom system.

forced vibration pass through the origin and are monotone because they are those of a vibration-in-unison; moreover, they must lie wholly in the $\varepsilon$-tubes because of (12.11). Consequently, the $L$-surface which is the locus of the endpoints of these trajectories, also lies inside the $\varepsilon$-tube.

The direction with which the trajectories pass through the origin is a function of the frequency $\omega$ of the exciting force as explained in connection with Figure 10. If that frequency lies near that of a normal-mode vibration, the trajectory lies near a modal line and is long. If not, the trajectory is, generally, short, and the amplitudes of the forced vibration are of $O(\varepsilon)$. In this way, one understands readily the occurrence of resonance.

It is also instructive to translate this information into the frequencyamplitude planes, shown in Figure 14. The dotted lines, composed of the $\omega$-axes and the backbone curves $[\mathbf{2 9}, \mathbf{3 8}]$, are those of every vibration-inunison of the autonomous system. In other words, if the autonomous system does not movein normal-mode vibrations, it is at rest with respect to admissible motions. (Because of Definition I, every vibration-in-unison of the autonomous system is a normal-mode vibration.)

Hence, we may surround the $\omega$-axes and the backbone curves by $\varepsilon$-tubes as shown in the diagram, and the forced steady-state vibrations of the nonautonomous neighboring system must be represented by response curves lying inside these $\varepsilon$-tubes. One such response curve, for a prescribed, small force-amplitude is shown. It is evident, then, that the intersections of 
$\omega$-axes and backbone curves are saddle points. In addition to these, there may be star points as well, if the steady-state forced vibrations are similar. If they are not, one can easily show that the only singular points in the

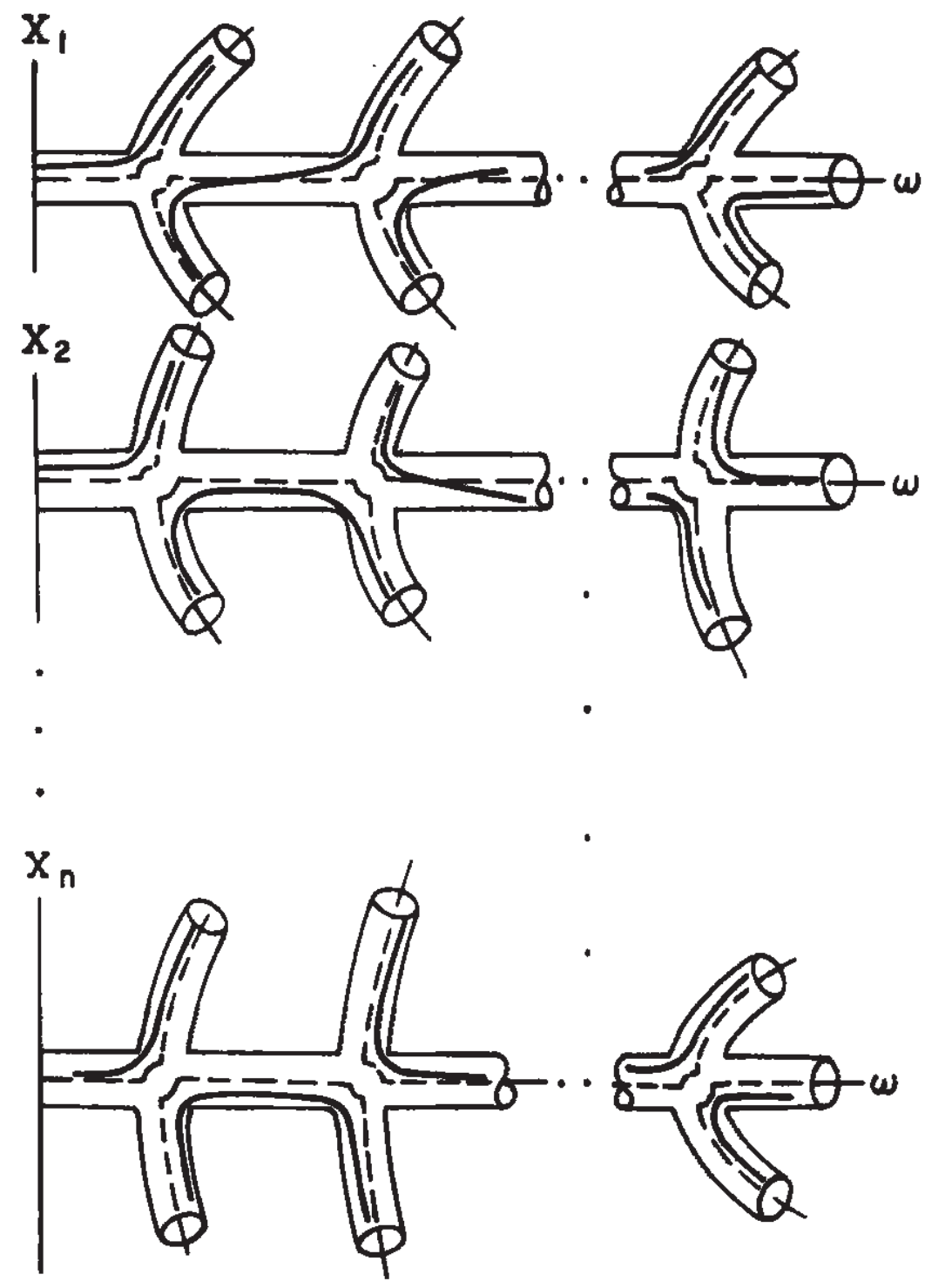

Fig. 14. Frequency-amplitude curves in nonlinear n-degree-of-freedom systems.

frequency-amplitude planes occur at the intersection of backbone curves with the $\omega$-axes, and all are saddles.

\section{Stability}

\section{Definitions}

Two definitions seem particularly useful in the examination of the stability of vibrations-in-unison: "stability in the sense of Liapunov," or L-stability and "stability in the sense of Poincare" or P-stability [39]. To define the former, let

$$
\left.\begin{array}{rl}
x_{i} & =x_{i}^{*}(t), \\
x_{i}^{*}(0) & =X_{i}, \quad \dot{x}_{i}^{*}(0)=0,
\end{array}\right\} \quad(i=1, \ldots, n),
$$


denote the set of solutions of a vibration-in-unison, satisfying the system $H$. Further, let

$$
x_{i}=x_{i}(t), \quad(i=1, \ldots, n),
$$

denote any set of solutions of the system $H$. Then, the set $x_{i}^{*}(t)$ is called $L$-stable if, for every $\varepsilon$, with $0<\varepsilon \ll X_{i},(i=1, \ldots, n)$, there exists a $\delta(\varepsilon)$, with $0<\delta(\varepsilon) \leqslant \varepsilon$, such that

$$
\left|x_{i}(t)-x_{i}^{*}(t)\right|<\varepsilon
$$

whenever

$$
\left|x_{i}(0)-x_{i}^{*}(0)\right|<\delta(\varepsilon) .
$$

To define $P$-stability, let

$$
\left.\begin{array}{rl}
x_{i} & =x_{i}^{*}\left(x_{1}\right), \\
x_{i}\left(X_{1}\right) & =X_{i},
\end{array}\right\} \quad(i=2, \ldots, n),
$$

denote the set of solutions of a trajectory corresponding to a vibration-inunison, and satisfying the system $M$. Further, let

$$
x_{i}=x_{i}\left(x_{1}\right), \quad(i=2, \ldots, n)
$$

denote any set of solutions of the system $M$. Then, the set $x_{i}^{*}\left(x_{1}\right)$ is called $P$-stable if, for every $\varepsilon$ with $0<\varepsilon \ll X_{i},(i=1, \ldots, n)$, there exists a $\delta(\varepsilon)$ with $0<\delta(\varepsilon) \leqslant \varepsilon$ such that

$$
\left|x_{i}\left(x_{1}\right)-x_{i}^{*}\left(x_{1}\right)\right|<\varepsilon
$$

whenever

$$
\left|x_{i}\left(X_{1}\right)-x_{i}^{*}\left(X_{1}\right)\right|<\delta(\varepsilon) .
$$

The interpretations of these two definitions of stability are clear. Let the space having coordinates $\left(x_{1}, \ldots, x_{n}, t\right)$ be called the event-space. Then $x_{i}^{*}(t),(i=1, \ldots, n)$ is a curve in the event-space. If any solution $x_{i}(t)$, having a single point inside an $\varepsilon$-tube about $x_{i}^{*}(t)$ remains within it everywhere, the solution is $L$-stable. Similarly, $x_{i}^{*}\left(x_{1}\right)$ is a curve in the configuration space. If any solution $x_{i}\left(x_{1}\right)$, having a single point inside an $\varepsilon$-tube about $x_{i}^{*}\left(x_{1}\right)$ remains within it everywhere, the solution is $P$-stable.* It is evident, therefore, that every $L$-stable solution is also $P$-stable, but the converse need not be true. Moreover, every solution that is $P$-unstable, is also $L$-unstable but, again, the converse need not be true.

- This type of stability is frequently called orbital stability [4]. 
In both definitions of stability it is required that $|\varepsilon|$ be small. Consequently it is sufficient to consider the equations of the first variation of $H$ with respect to $x_{i}^{*}(t)$; i.e.,

$$
\ddot{\xi}_{i}=\sum_{i=1}^{n} \xi_{i} \frac{\partial^{2}}{\partial \xi_{i} \partial \xi_{j}}\left[U\left(x_{1}^{*}(t), \ldots, x_{n}^{*}(t)\right)\right], \quad(i=1, \ldots, n) .
$$

Clearly, this is a system of linear equations with periodic coefficients, and stability in any sense requires that all characteristic exponents of (13.9) have real negative parts. Now, the number of characteristic exponents depends on whether the system $H$ is an explicit function of $t$, or not; i.e., whether the system $H$ is autonomous or not [26]. Hence, the two cases of normal-mode vibrations and of steady-state forced vibrations must be discussed separately.

\section{Stability of Normal-Mode Vibrations}

It is simple to deduce [21]

Theorem XIII-I: If an admissible potential function $U$ is not a quadratic form, the normal-mode vibrations are never $L$-stable.

This result is an immediate consequence of the well-known fact that the periodic solutions (if they exist) of autonomous, nonlinear second-order equations are not isochronous. It follows from this theorem that normalmode vibrations of nonlinear systems are, at most, $P$-stable, and their stability properties depend in general, not only on the magnitudes of the mass and spring parameters, but on the energy level of the motion as well.

The problem of discussing the behavior of the $\xi_{i}$ in (13.9) is greatly simplified when that system of coupled equations can be decoupled. If the system (13.9) is written in the matrix form

$$
\ddot{\xi}+B(t) \xi=0
$$

it turns out that the matrix $B(t)$ is of the form

$$
B(t)=B_{0}+\sum_{i=1}^{m} f_{i}(t) B_{i}
$$

where $B_{0}$ and the $B_{i}$ are square matrices with constant elements, and the $f_{i}(t)$ are periodic scalar functions of time. Then, if a transformation matrix $T$ exists such that

$$
T^{-1} B(t) T=\Lambda(t)
$$


where $\Lambda(t)$ is a diagonal matrix, the transformed system (13.9) is decoupled. Hsu has shown $[40]$ that the conditions, necessary and sufficient for the existence of such a transformation matrix $T$ is, that the $B_{i},(i=0, \ldots, m)$ be all commutative and that at least one of them have distinct eigenvalues.

\section{Homogeneous Systems}

It is simple to demonstrate [7]

Theorem XIII-II: The system of variational equations of admissible, homogeneous systems with respect to any normal-mode vibration can always be decoupled.

To prove it, one shows that, in homogeneous systems, (13.11) can always be reduced to

$$
B(t)=A \sum_{i=1}^{m} f_{i}(t)
$$

where $A$ is a constant, square matrix. Thus, Hsu's criterion is trivially satisfied.

The normal-mode vibrations of homogeneous systems have the further (unusual) property that their stability does not depend on the energy level of the motion. In this respect, they are similar to linear systems, the latter being stable at all amplitudes. If a normal-mode vibration of a homogeneous system is stable (unstable) at any one amplitude, it is stable (unstable) at all amplitudes [9]. Whether a given normal mode vibration of a given homogeneous system is stable or not depends only on the mass and spring parameters of the system, and on the particular normal mode examined, but not on the energy level.

As an example, consider a homogeneous system having the degrees of freedom $u$ and $v$. Then, the equations of motion may be written in the form suggested by Loude [41]:

$$
\begin{aligned}
& m_{1} \ddot{u}=-a \psi(u)-A \psi(u-v), \\
& m_{2} \ddot{v}=-b \psi(v)+A \psi(u-v)
\end{aligned}
$$

where

$$
\psi(w) \equiv w|w|^{k-1} .
$$

If the trajectory of a given normal mode is

$$
v=c u
$$


i.e.,

$$
\begin{aligned}
& u=\varphi(t, X), \\
& v=c \varphi(t, X),
\end{aligned}
$$

one can show that the variational equations have solutions that satisfy [41]

$$
\eta=B \xi, \quad\left(\xi_{1}=\xi, \quad \xi_{2}=\eta\right)
$$

where $B$ takes on one or the other of the two values

$$
\begin{aligned}
& B_{1}=c, \\
& B_{2}=-m_{1} / c m_{2} .
\end{aligned}
$$

The variational equations reduce to

$$
\ddot{\xi}+D_{1,2} \psi^{\prime}(\varphi) \xi=0
$$

where $D_{1,2}$ are constants defined by

$$
\begin{aligned}
& D_{1}=\frac{a}{m_{1}}+\frac{A}{m_{1}}(1-c)|1-c|^{k-1}, \\
& D_{2}=\frac{a}{m_{1}}+\frac{A}{m_{1}}\left(1+\frac{m_{1}}{c m_{2}}\right)|1-c|^{k-1} .
\end{aligned}
$$

It is easy to show that these equations are independent of the amplitude $X$. To do this, we note that the motion in normal modes is an ateb-function. (The arguments which follow are the same, whether we deal with sam- or cam-functions; thus, only one of these need be considered.) From (9.6) and (9.34),

$$
\begin{aligned}
& x=X \operatorname{cam}(n \tau), \\
& \tau=(c / n)^{1 / 2} X^{n-1} t .
\end{aligned}
$$

But, the independent variable in $(13.20)$ is $t$, not $\tau$. Now, one has from (13.22)

$$
\frac{d^{2}}{d t^{2}}=E X^{k-1} \frac{d^{2}}{d \tau^{2}}
$$

where $E$ is a constant not containing $X$, and from the definition (13.15) of the $\psi$-function together with (13.22),

$$
\psi^{\prime}(\varphi)=X^{k-1} \psi^{\prime}(\operatorname{cam}(n \tau)) .
$$


Hence, (13.20) in terms of $\tau$, becomes

$$
\frac{d^{2} \xi}{d \tau^{2}}+D_{1,2} \psi^{\prime}(\operatorname{cam}(n \tau)) \xi=0
$$

where $D_{1,2}=D_{1,2} / E$. It follows that the two equations (13.25) do not contain the amplitude $X$.

The discussion of the characteristic exponents of the equations (13.25) is difficult because they are Hill equations in which the periodic coefficients are cam-functions. The detailed stability properties of such Hill equations are not known.

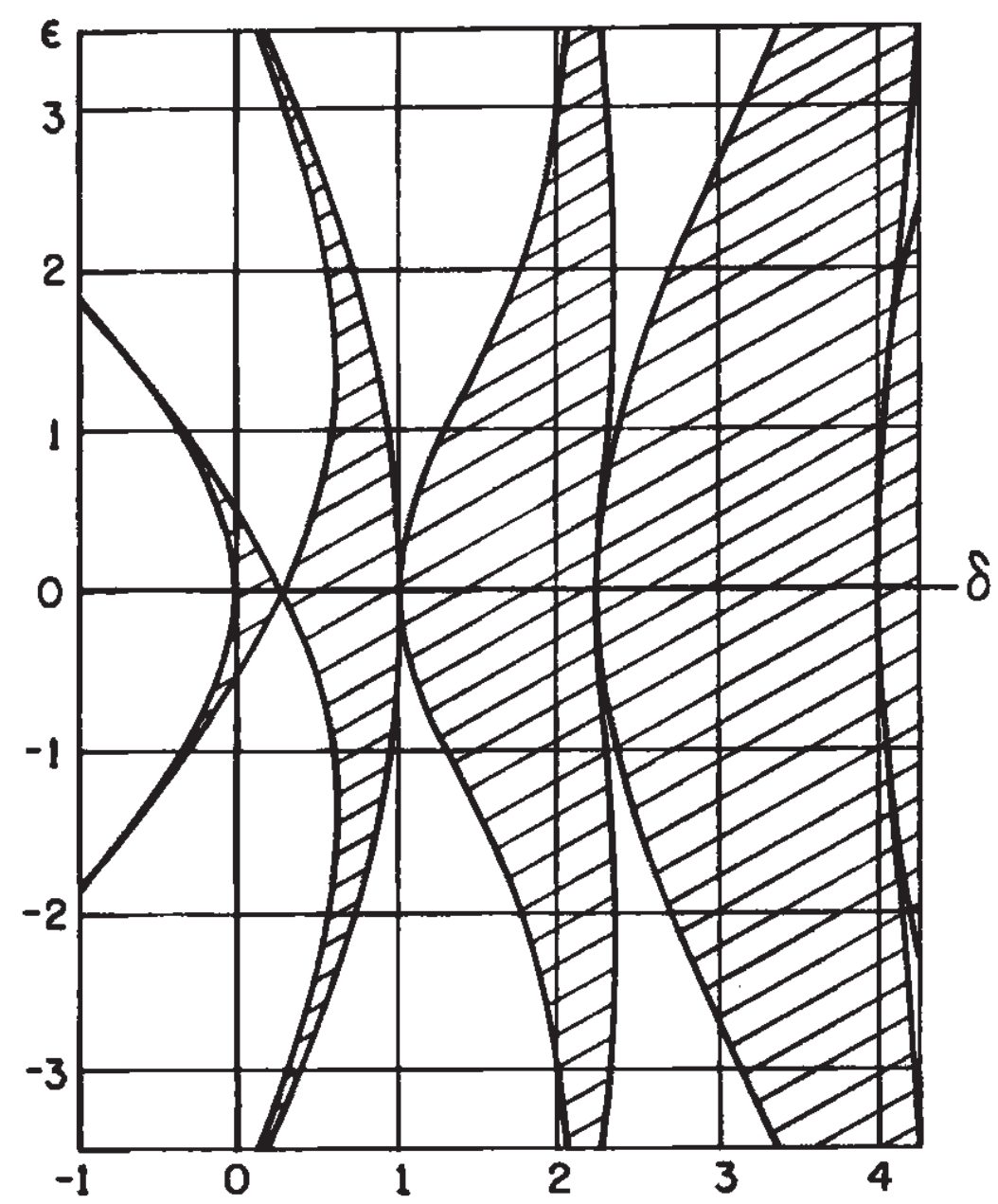

Fig. 15. Strutt chart.

However, one can gain some insight into the stability of normal-mode vibrations of homogeneous systems by replacing the cam-function in (13.25) by a cosine-function. Proceeding in this manner is, in fact, equivalent to expanding the periodic coefficient into Fourier series and retaining only the first term of the expansion. This is usually done in examining the details of stability of vibratory motions of nonlinear systems [4]. It has the consequence that equations (13.25) become Mathieu equations whose stability properties are well known $[4,42]$. 
One finds, in place of (13.25), two Mathieu equations of the form

$$
\xi^{\prime \prime}+\left(\delta_{1,2}+\varepsilon_{1,2} \cos \tau\right) \xi=0
$$

and for stability of the normal-mode vibration, it is necessary, that both these equations "indicate stability."

The stability study is carried out by means of the Strutt chart [4]. This chart consists of a set of curves in the $\varepsilon \delta$-plane separating stable regions (shaded) from unstable ones; it is shown in Figure 15. If the values $P_{1}\left(\delta_{1}, \varepsilon_{1}\right)$ and $P_{2}\left(\delta_{2}, \varepsilon_{2}\right)$ define two points $P_{1}$ and $P_{2}$ in the $\varepsilon \delta$-plane which lie in the interior, or on the boundary, of a stable region, the motion is stable. If one or both of these points lie in the interior of an unstable region, the motion is unstable. It turns out that the values of the parameters $\varepsilon, \delta$ in one of the equations (13.26) is such that one of the points $P(\varepsilon, \delta)$ always lies on a boundary between stable and unstable regions. This is known to lead, at most, to $P$-stability [4], thus verifying Theorem XIII-I. The other point may lie anywhere in the $\varepsilon \delta$-plane.

The results of the computation for

$$
B=B_{1}=c
$$

are given below for that one of the two Mathieu equations for which $P(\varepsilon, \delta)$ does not automatically lie on a boundary.

That equation is

$$
\xi^{\prime \prime}+(\delta+\varepsilon \cos \tau) \xi=0
$$

where [9]

(13.29)

$$
\left\{\begin{array}{l}
\delta=\alpha \delta / 4, \quad \varepsilon=\alpha \bar{\varepsilon} / 4, \\
\delta=2^{-(k-2)}\left\{\frac{1}{2} \frac{\Gamma(k)}{\left[\Gamma\left(\frac{k+1}{2}\right)\right]^{2}}\right\}, \\
\bar{\varepsilon}=2^{-(k-2)}\left\{\frac{1}{2} \frac{\Gamma(k)}{\Gamma\left(\frac{k+3}{2}\right) \Gamma\left(\frac{k-1}{2}\right)}\right\}, \\
\alpha=\frac{2}{\pi^{2}} \frac{\beta}{c} \frac{k+1}{2}\left\{\frac{\Gamma\left(\frac{1}{k+1}\right) \Gamma\left(\frac{1}{2}\right)}{\Gamma\left(\frac{1}{k+1}+\frac{1}{2}\right)}\right\}^{2}, \\
\beta=\frac{k}{m_{1}}\left[a+A|1-c|^{k-1}(1-c)\right] .
\end{array}\right.
$$


For any given system parameters, the formulas (13.29) can be utilized to determine the location of $P(\delta, \varepsilon)$. Similar results are found, when

$$
B=B_{2}=-m_{1} / c m_{2} .
$$

In either case, it turns out that the point $P(\varepsilon, \delta)$ lies on a straight line in the $\varepsilon \delta$-plane which passes through the origin, which lies always in the right half-plane, and whose slope is a function of $k$ only. When $k=1$, this slope is zero so that the point lies then on the (positive) $\delta$-axis which consists entirely of stable points. Thus, linear systems are always stable. When $k>1$, the slope of the straight line is positive, and when $0<k<1$, the slope is negative. The location of $P(\delta, \varepsilon)$ on the straight line is a function of the system parameters [28].

\section{Symmetric System with Two Degrees of Freedom [9]}

As a second example permitting decoupling of the variational equations, consider the symmetric two-degree-of-freedom system (not homogeneous) [9]. The equations of the pseudo-system are

$$
\begin{aligned}
& \ddot{x}=U_{x}(x, y), \\
& \ddot{y}=U_{y}(x, y),
\end{aligned}
$$

and the trajectories of the normal modes are

$$
y=y_{i}^{*}(x)=x ; \quad y=y_{0}^{*}(x)=-x
$$

where the subscripts $i$ and $o$ indicate an in-phase mode and an out-of-phase mode, respectively. Using the notation

$$
Q\left(x^{*}(t), y^{*}(t)\right)=Q^{*}
$$

for $U$ and its derivatives, it is easily shown that, in symmetric systems, one has always

$$
U_{x x}^{*}=U_{y y}^{*}
$$

for both, the $i$ and $o$-modes.

The coupled variational equations are

$$
\begin{aligned}
& \ddot{\xi}-U_{x x}^{*} \xi-U_{x y}^{*} \eta=0, \\
& \ddot{\eta}-U_{x y}^{*} \xi-U_{y y}^{*} \eta=0 .
\end{aligned}
$$

Under the transformations

$$
\begin{aligned}
& \zeta_{1}=\eta+\xi, \\
& \zeta_{2}=\eta-\xi,
\end{aligned}
$$


the equations (13.35) become, in virtue of (13.34),

$$
\begin{aligned}
& \ddot{\zeta}_{1}-\left(U_{x z}^{*}+U_{x y}^{*}\right) \zeta_{1}=0, \\
& \ddot{\zeta}_{2}-\left(U_{y y}^{*}+U_{x y}^{*}\right) \zeta_{2}=0,
\end{aligned}
$$

and these are, evidently, decoupled.

As a specific example, consider the system ${ }^{\dagger}$

$$
U=U^{(L)}+U^{(N)}
$$

where

$$
\begin{aligned}
& U^{(L)}=-\frac{1}{2} a_{1}\left(x^{2}+y^{2}\right)-\frac{1}{2} A_{1}(x-y)^{2}, \\
& U^{(M)}=-\frac{1}{1} a_{3}\left(x^{4}+y^{4}\right)-1 A_{3}(x-y)^{4} .
\end{aligned}
$$

We now introduce the following notation, already indicated, in part, in (13.33): when $U$ or its partial derivatives are evaluated along a modal line, that quantity is marked with *. When, its value along such a modal line is computed at the values $X, y(X)=Y$, the quantity is marked with **. If the modal line in question is that of the in-phase mode, the quantity is supplied with an $i$-subscript, if it is that of the out-of-phase mode, the quantity has an 0 -subscript. If no subscript is supplied the quantity is the same in the two modes.

With this notation we define:

$$
\begin{aligned}
& P^{(L)}=\frac{1}{2}\left[\left(U_{x x}^{(L)}\right)^{* *}+\left(U_{y y}^{(L)}\right)^{* *}\right], \\
& P_{i, 0}^{(N)}=\frac{1}{2}\left[\left(U_{x x}^{(N)}\right)_{i, 0}^{* *}+\left(U_{y y}^{(N)}\right)_{i, 0}^{* *}\right], \\
& Q^{(L)}=\left(U_{x y}^{(L)}\right)^{* *}, \\
& Q_{i, 0}^{(N)}=\left(U_{x y}^{(N)}\right)_{i, 0}^{* *} .
\end{aligned}
$$

Note that the quantities arising from the linear part of the potential function are the only ones independent of the mode.

It will be remembered from the earlier example that the nonlinear vibrations $x^{*}(t), y^{*}(t)$ will be replaced, in the variational equations, by their zeroth approximation; i.e., by simple harmonic functions. It is entirely consistent with this approximation, to replace the frequencies of the normalmode vibrations by their Duffing approximations [4]. These are, in terms of the quantities defined in (13.40)

t The superscripts $(L)$ and $(N)$ indicate the terms in $U$ which give rise, respectively, to linear and to nonlinear terms in the equations of motion. 


$$
\begin{aligned}
& \omega_{i}^{2}=-\left(P^{(L)}+Q^{(L)}\right)-1\left(P_{i}^{(N)}+Q_{i}^{(N)}\right), \\
& \omega_{0}^{2}=-\left(P^{(L)}-Q^{(L)}\right)-\frac{1}{(}\left(P_{0}^{(N)}-Q_{0}^{(N)}\right) .
\end{aligned}
$$

If one utilizes

$$
\begin{aligned}
\cos ^{2} \omega_{i, 0} t & =\frac{1}{2}+\frac{1}{2} \cos 2 \omega_{i, 0} t_{,} \\
2 \omega_{i, 0} t & =\tau_{i, 0},
\end{aligned}
$$

the equations of the first variation become

$$
\zeta_{1,2}^{\prime \prime}+\left(\delta_{1,2}^{i, 0}+\varepsilon_{1,2}^{i, 0} \cos \tau_{i, 0}\right) \zeta_{1,2}=0
$$

where primes denote differentiation with respect to $\tau_{i, 0}$, corresponding super- and subscripts must be used, and

$$
\delta_{1,2}^{i, 0}=-\frac{1}{4 \omega_{i, 0}^{2}}\left[\left(P^{(L)} \pm Q^{(L)}\right)+\frac{1}{2}\left(P_{i, 0}^{(N)} \pm Q_{i, 0}^{(N)}\right)\right]
$$

$$
\varepsilon_{1,2}^{i, 0}=-\frac{1}{4 \omega_{i, 0}^{2}}\left[\frac{1}{2}\left(P_{i, 0}^{(N)} \pm Q_{i, 0}^{(N)}\right)\right]
$$

or, combining them,

$$
\delta_{1,2}^{i, 0}=-\frac{1}{4 \omega_{i, 0}^{2}}\left(P^{(L)} \pm Q^{(L)}\right)+\varepsilon_{1,2}^{i, 0}
$$

In (13.44) and (13.45), the + signs are used with the subscript 1 , and the - signs with the subscript 2.

Evidently then, (13.43) represents four equations, those for two modes, and two equations for each mode. As in the earlier example, one of the two equations in each mode defines points in the Strutt chart which lie always on the boundary between stable and unstable regions of the $\varepsilon \delta$-plane. Hence, it is the other two equations which determine the stability; these are the combinations $(1,0)$ and $(2, i)$.

In applying the foregoing formulas to the example of potential function (13.38) and (13.39), it is convenient to introduce the nondimensional quantities

$$
\begin{array}{rlrl}
\alpha_{1} & =A_{1} / a_{1} ; & \alpha_{8} & =A_{8} / a_{8} ; \\
v_{i}^{2} & =\omega_{i}^{2} / a_{1} ; & v_{0}^{2}=\omega_{0}^{2} / a_{1} ; \\
E_{i}=\frac{3}{4} \frac{a_{3}}{a_{1}} X_{i}^{2} ; & E_{0}=\frac{3}{4} \frac{a_{8}}{a_{1}} X_{0}^{2} .
\end{array}
$$


In terms of these quantities, the parameters become, for the $i$-mode,

$$
\delta_{2}^{i}=\frac{1+2 \alpha_{1}}{4\left(1+E_{i}\right)}+\varepsilon_{2}^{i}
$$

$$
\varepsilon_{2}^{i}=\frac{1}{2} \frac{E_{i}}{1+E_{i}}
$$

and a similar result in the 0 -mode. In both cases, the parameters $\varepsilon, \delta$ depend on $E$; i.e., on the amplitude of the motion.

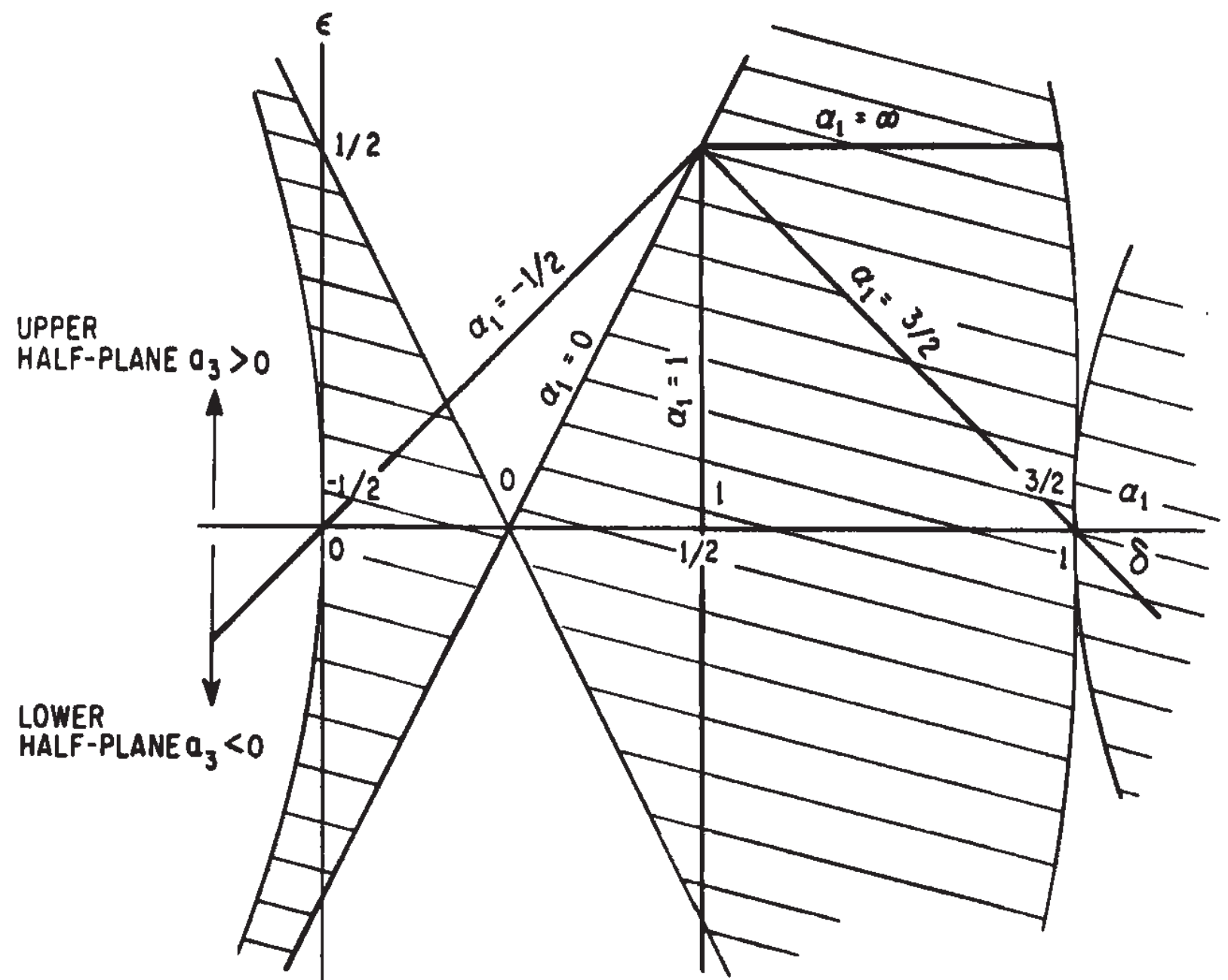

FIG. 16. Stability of in-phase mode of nonlinear, symmetric system with two degrees of freedom.

The results for the $i$-mode are shown in Figure 16 and are as follows:

(i) The point on the Strutt chart, deciding on the stability of the $i$ mode lies on a straight line passing through the point $\left(\frac{1}{2}, \frac{1}{2}\right)$, and intersecting the positive $\delta$-axis at a point that depends on $\alpha_{1}$ only. This straight line is called a stability characteristic.

(ii) The portion of the stability characteristic lying in the upper halfplane applies when $a_{3}>0$, that in the lower half-plane when $a_{3}<0$. 
(iii) The location of the point on a stability characteristic is a function of the amplitude; it lies on the $\delta$-axis for zero amplitude, and its distance from the $\delta$-axis increases with amplitude. Hence, for small amplitudes, the point deciding on the stability lies in the neighborhood of the $\delta$-axis, and that neighborhood consists almost everywhere of stable points. One may conclude that, in general, normal mode vibrations of small amplitude are stable.

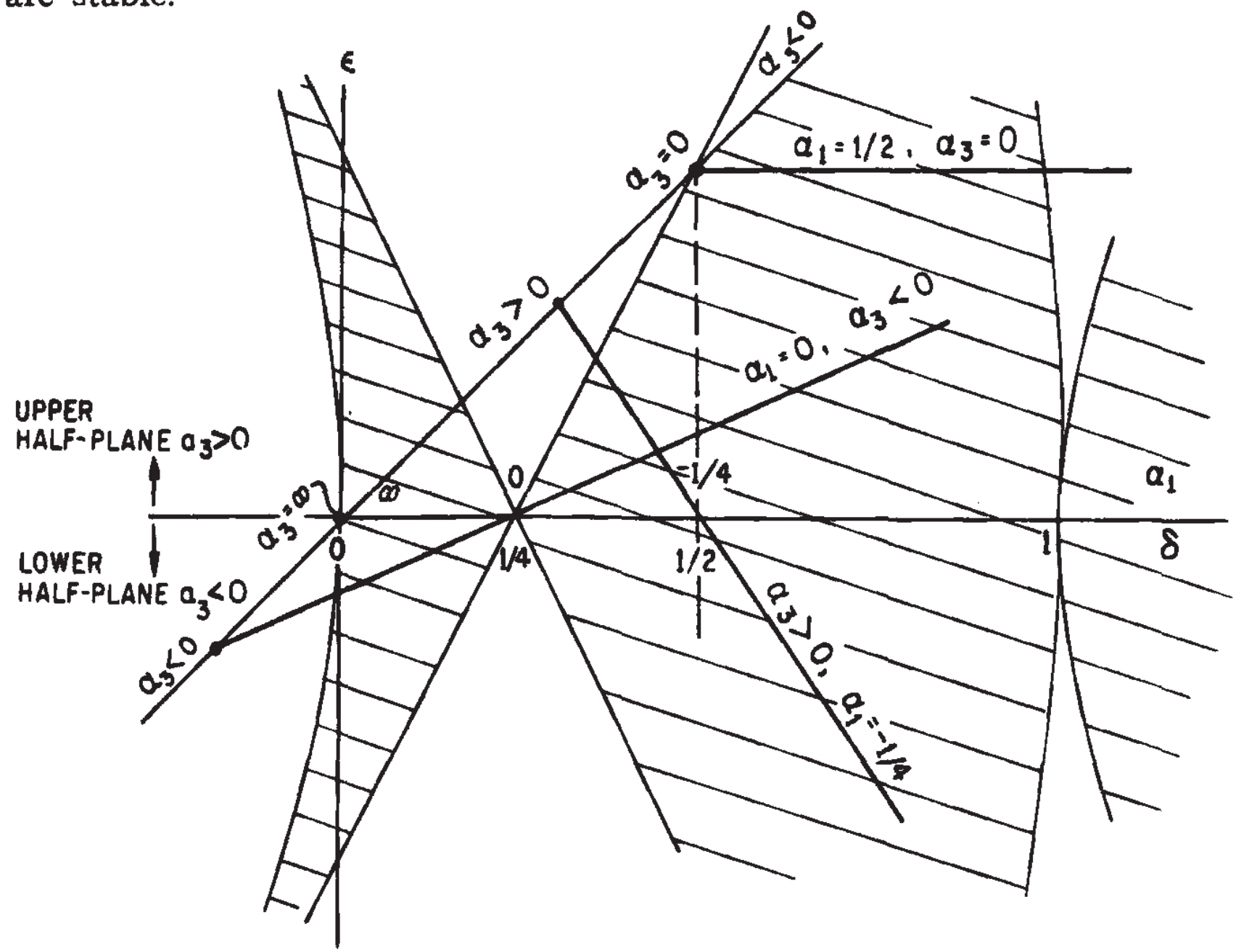

FIG. 17. Stability of out-of-phase mode of nonlinear, symmetric system with two degrees of freedom.

The results for the o-mode are shown in Figure 17. They differ in some respects from those for the $i$-mode.

(i) There exist, again, straight stability characteristics. These terminate on a point lying on a straight line of unit slope, passing through the origin. The point on this line, at which the stability characteristics terminate, depends on $\alpha_{3}$ only. Further, the stability characteristics intersect the positive $\delta$-axis at a point whose value depends on $\alpha_{1}$ only. Thus, knowledge of $\alpha_{1}$ and $\alpha_{3}$ determines the stability characteristic and the amplitude of the motion determines the point on the stability characteristic. Items (ii) and (iii), listed above for the $i$-mode also apply to the 0 -mode. From these results, knowledge of $\alpha_{1}, \alpha_{3}, a_{3}$ and the amplitude of the motion permits the determination of its stability. 
We shall consider here the stability of a nonsimilar normal-mode vibration of an autonomous system that neighbors on a parent system of potential function

$$
U=U\left(x_{1}, \ldots, x_{n}\right)
$$

of equations of motion

$$
\ddot{x}_{i}=U_{x_{i}}\left(x_{1}, \ldots, x_{n}\right), \quad(i=1, \ldots, n)
$$

and having a similar normal-mode vibration

$$
x_{i}=x_{i}^{*}(t), \quad(i=1, \ldots, n) .
$$

Let the perturbed system have the admissible potential function

$$
0=U\left(x_{1}, \ldots, x_{n}\right)+\varepsilon \omega\left(x_{1}, \ldots, x_{n}\right), \quad(|\varepsilon| \ll|U|) .
$$

The equations of motion of the perturbed system are

$$
\ddot{x}_{i}=U_{x_{i}}\left(x_{1}, \ldots, x_{n}\right)+\varepsilon \omega_{x_{i}}\left(x_{1}, \ldots, x_{n}\right), \quad(i=1, \ldots, n),
$$

and it has been shown in a previous section that this system has a normalmode vibration (in general nonsimilar) of the form

$$
\bar{x}_{i}(t)=x_{i}^{*}(t)+\varepsilon \xi_{i}(t)+\ldots, \quad(i=1, \ldots, n),
$$

Then, one can easily demonstrate [8].

Theorem XIII-III: The stability of the normal-mode vibration (13.53) of the perturbed system is the same as the stability of the similar normal-mode vibration (13.50) of the parent system.

For the proof, one forms the equations of the first variation of (13.62) with respect to the solution (13.53). It turns out that these are precisely the same equations as those of the first variation of the equations of motion of the parent system with respect to the solutions (13.50), which proves the theorem. Hence, if the variational equations of the parent system can be decoupled, the stability problem of normal-mode vibrations of neighboring systems is solved.

\section{Forced Vibrations}

In general, the variational equations of the equations of motion with respect to a vibration-in-unison cannot be decoupled, even when the trajectories of the motion in configuration space are straight. In these cases, Hsu has developed criteria for determining the stability $[43,44]$; his results 
are based on the application of the method of Strubel [39] in a slightly modified form. A comprehensible summary of Hsu's methods and results is too lengthy to be reproduced here. However, we shall apply his method to a particular case of steady-state forced vibrations in which the variational equations cannot be decoupled; this example is due to Kinney [12].

We consider the nonautonomous, admissible system

$$
\begin{aligned}
& \ddot{x}_{1}=\hat{O}_{x_{1}}\left(x_{1}, \ldots, x_{n}\right)+f(\omega t), \\
& \ddot{x}_{j}=\sigma_{x_{j}}\left(x_{1}, \ldots, x_{n}\right) ; \quad(j=2, \ldots, n),
\end{aligned}
$$

where

$$
0=U\left(x_{1}, \ldots, x_{n}\right)+\varepsilon \psi\left(x_{1}, \ldots, x_{n}\right)
$$

and

$$
U=U\left(x_{1}, \ldots, x_{n}\right)
$$

is a negative quadratic form. In other words, the parent system is a linear admissible, nonautonomous system in which the mass $m_{1}$ is subjected to periodic forcing of period

$$
T=2 \pi / \omega .
$$

We inquire whether a vibration-in-unison of the perturbed system, given by

$$
x_{i}=x_{i}^{*}(t), \quad(i=1, \ldots, n)
$$

is stable.

The equations of the first variation of (13.54) with respect to (13.68) are

$$
\ddot{z}_{i}=\sum_{k=1}^{n}\left[\left(\frac{\partial^{2} U}{\partial x_{i} \partial x_{k}}\right)^{*}+\varepsilon\left(\frac{\partial^{2} \psi}{\partial x_{i} \partial x_{k}}\right)^{*}\right] z_{i}, \quad(i=1, \ldots, n),
$$

where use was made of notation (13.33) with respect to the second derivatives of $U$ and $\psi$. Since $U$ is a quadratic form, the $\partial^{2} U / \partial x_{i} \partial x_{k}$ are constants for all $i$ and $k$.

We write (13.59) in the matrix form

$$
\ddot{z}+\left[B_{0}+\varepsilon B(t)\right] z=0
$$

where $z$ is a column matrix, $B_{0}$ is a constant square matrix,

$$
B(t)=\sum_{s=1,2, \ldots}^{S} B_{s} \cos s \omega t,
$$

and the $B_{s}$ are constant square matrices. 
It will be noticed that, here, we have not made the assumption that the solutions (13.58) are to be treated as simple harmonic for purposes of a stability analysis. In fact, that assumption is unnecessary here because the approach through Mathieu equations is not used.

It is now supposed that $B_{0}$ and the $B_{s}$ do not satisfy Hsu's criterion [40] and that, in consequence, (13.59) cannot be decoupled.

We introduce the transformation

$$
z=T \xi
$$

which is such that $T$ is a constant matrix that diagonalizes $B_{\mathbf{0}}$. Under it, (13.60) goes over into a matrix equation of the form

$$
\ddot{\xi}+\left(\Omega+\varepsilon \sum_{s=1}^{s} D_{s} \cos s \omega t\right) \xi=0
$$

where $\Omega$ is a diagonal matrix with elements

$$
\omega_{1}^{2} \leqslant \omega_{2}^{2} \leqslant \ldots \leqslant \omega_{n}^{2},
$$

and the

$$
D_{s}=\left[d_{i j}^{(s)}\right], \quad(s=1, \ldots, S)
$$

are constant matrices.

Then, Hsu's results show that instability occurs when, and only when, the following inequalities are satisfied:

$$
\begin{gathered}
{\left[s \omega-\left(\omega_{k}+\omega_{j}\right)\right]^{2}<\frac{\varepsilon^{2}}{4} \frac{d_{k j}^{(s)} d_{j k}^{(s)}}{\omega_{k} \omega_{j}},} \\
{\left[s \omega-\left(2 \omega_{k}\right)\right]^{2}<\frac{\varepsilon^{2}}{4} \frac{\left(d_{k k}^{(s)}\right)^{2}}{\omega_{k}^{2}}} \\
{\left[s \omega-\left(\omega_{j}-\omega_{k}\right)\right]^{2}<-\frac{\varepsilon^{2}}{4} \frac{d_{k j}^{(s)} d_{j k}^{(s)}}{\omega_{k} \omega_{j}}}
\end{gathered}
$$

Now, the functional relation between the frequency $\omega$ of the exciting function and the amplitude $X_{1}$ of $x_{1}{ }^{*}(t)$ is known. Hence, the inequalities (13.66) determine the amplitudes $X_{1}$ (if any) for which the motion is unstable.

Kinney [12] has applied this theory to the weakly nonlinear problem of the dynamic vibration absorber. The analysis is too detailed and tedious to be reproduced here because of the involved form of the matrix elements $d_{i j}^{(s)}$. His result for that problem is:

The portions of the response curves (in the frequency-amplitude planes) which have negative slope correspond to unstable motion. All other portions of these curves correspond to stable motion. 
On the Existence of Simple Trajectories in Admissible $n$-Degree-of-Freedom Systems*

In this appendix we employ

Definition A-I: An integral of the autonomous system $H(3.3)$ or of the system $M(3.12)$ is said to be a simple trajectory in configuration space if and only if:

(i) it passes through the origin;

(ii) it reaches the bounding, or maximum equipotential surface (5.8);

(iii) it has no tangency with any equipotential surface (5.12) except at the origin and the bounding surface.

The last restriction was not imposed by Kuo [18] who introduced the concept of the simple trajectory. But the lack of tangency with any equipotential surfaces $U+h^{*}=0,0<h^{*}<h$ is a property of modal lines. In fact, every modal line is a simple trajectory, and every simple trajectory which satisfies (i) to (iii) as well as

$$
\frac{d x_{i}}{d x_{1}} \neq 0, \quad(i=1, \ldots, n)
$$

for all $x_{1}$, is a modal line.

With the above definition we prove in this appendix the central

Theorem A-I: Every admissible autonomous system possesses at least one simple trajectory.

Before presenting the proof of this theorem it may be helpful to sketch its essential ideas; these are very similar to those used in the two-dimensional problem [19].

We consider trajectories in configuration space which pass through (or issue from) a point $P_{0}$ on the bounding surface; such trajectories are called $T$-curves. We then demonstrate that every $T$-curve passes through an infinity of isolated distinct points $Q_{i}(i=1,2, \ldots)$, corresponding to configurations of stationary potential energy with respect to neighboring points on $T$. The corresponding levels of potential energy are denoted by $h_{j}$, and the transit times through the $Q_{j}$ by $t_{j}$. We show, further, that the $t_{j}$ and $h_{i}$ are uniquely determined by the initial point $P_{0}$, and both are differentiable with respect to $P_{0}$. Next, we demonstrate that there exists at least one $T=T^{*}$, issuing from a point $P_{0}^{*}$ for which the ordered $Q_{i}(i=1,2, \ldots)$ lie alternately at the origin and at $P_{0}^{*}$. Finally, we show that $T^{*}$ satisfies condition (iii) above; this completes the proof.

- This appendix is based in large part on work by J. K. Kuo [18] and by C. H. Pak. 
We use the following notation:

$q=\left(q_{1}, \ldots, q_{n}\right)$ is an $n$-dimensional physical displacement vector;

$\dot{q}=\left(\dot{q}_{1}, \ldots, \dot{q}_{n}\right)$ is an $n$-dimensional physical velocity vector;

$K=1 / 2 \sum_{i=1}^{n} m_{i} \dot{q}_{i}^{2}$ is the kinetic energy of the physical system;

$-\hat{O}=-\hat{O}(q) \quad$ is the potential energy stored in the springs;

$h=K-O \quad$ is the total energy of the physical system;

$\nabla U=\operatorname{grad} U \quad$ is an $n$-dimensional vector.

We assume that 0 satisfies the following conditions, called "admissibility conditions":

(i) $\delta(q)=\delta(-q) \quad$ is of class $C^{2}$;

(ii) $-0 \quad$ is positive definite;

(iii) $\nabla O=0 \quad$ only at $q=0$.

(iv) Trajectories are the vectors $q(t)$ which satisfy the equations of motion,

$$
m_{i} \ddot{q}_{i}=\frac{\partial}{\partial q_{i}} O(q), \quad(i=1, \ldots, n)
$$

where the $m_{i}$ are real, positive constants. The equations of motion transform under

$$
x_{i}=\sqrt{m_{i}} q_{i}
$$

into

$$
\ddot{x}_{i}=\frac{\partial}{\partial x_{i}} U(x)=U_{, i}
$$

or, in vector notation,

$$
\ddot{x}=\nabla U \text {. }
$$

With Kuo [18], we transform (A.3) into the system of $2 n$ first-order equations

$$
\dot{y}_{i}=f_{i}(y)
$$

where

$$
\begin{array}{ll}
y_{i}=x_{i}, & y_{i+n}=\dot{x}_{i}, \\
f_{i}=y_{n+i}, & f_{i+n}=U_{n} .
\end{array}
$$


Since the system is admissible, the functions $f_{i}$ are of class $C^{1}$, or the Jacobian $J$ is continuous, where

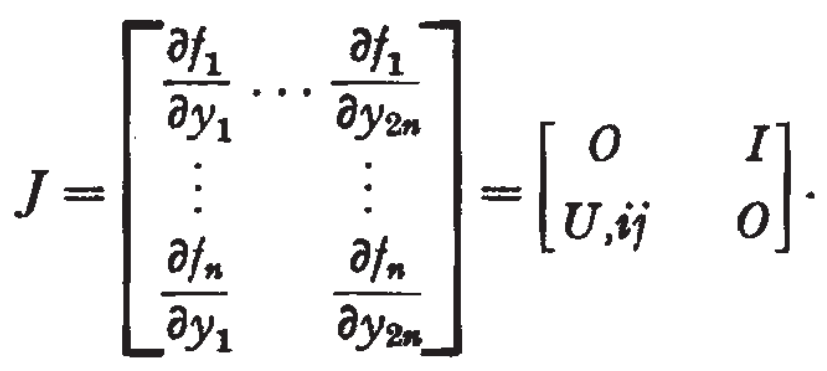

In consequence, (A.4) has a unique solution under specified initial conditions: $y=Y$ when $t=\tau$; that solution is

$$
y=y(t, \tau, Y), \quad-\infty<t<+\infty .
$$

Conversely, if the initial condition $Y=y$ at $\tau=t$ is given, one has uniquely

$$
Y=Y(\tau, t, y), \quad-\infty<\tau<+\infty .
$$

Finally, since $J$ is of class $C^{1}$, the functions $y$ and $Y$ in (A.6) and (A.7) are once differentiable with respect to all arguments. If one regards (A.6) as a mapping which, for fixed $t$, maps the initial point $Y$ onto $y$, and (A.7) as the inverse of this mapping, one notes that this mapping and its inverse are continuous, or (A.4) is a topological mapping in the phase space. Clearly, the energy integral of (A.4) exists and is

$$
-U+\frac{1}{2}\|\dot{x}\|^{2}=h=\text { const }
$$

where \|\| denotes the Euclidean distance function, $h \geqslant 0$ is the total energy of the system, and it is bounded. The configuration of maximum potential energy is

$$
U+h=0 .
$$

This is the maximum equipotential surface and it is the bounding surface for all possible trajectories of admissible systems (Theorem V-II).

All trajectories satisfy

Lemma A-I: No integral curve of (A.4) terminates at a point.

Lemma A-II: If an integral curve of (A.4) passes through $(X, X)$ at $t=0$ and it reaches the bounding surface at $t=t_{0}$, then it passes through $(X,-X)$ at $t=2 t_{0}$.

The proofs of these lemmas are simple and will only be indicated. To prove the first, one supposes to the contrary that a trajectory does terminate at a point $x$ when $t=\tau_{0}$. Then, for all $t>\tau_{0}, \dot{x}=\ddot{x}=\ldots=0$. But, when $\dot{x}=0$, the configuration is that of maximum potential energy, and when $\ddot{x}=0, x$ is at the origin. Since the origin does not lie on the bounding surface under our admissibility condition, Lemma A-I is true. 
For proof of the second lemma one need only observe that trajectories which attain the bounding surface intercept that surface orthogonally (Theorem V-V). Hence, the initial conditions (position and slope) are defined; then, in view of the uniqueness of (A.6), the trajectory must retrace itself.

It is now convenient to rewrite the energy integral in terms of the initial energy when the initial point is not on the bounding surface:

$$
-U(x)+\frac{1}{2}\|\dot{x}\|^{2}=-U(X)+\frac{1}{2}\|\dot{X}\|^{2}
$$

where $x$ and $\dot{x}$ as functions of the initial values $X$ and $\dot{X}$ are given in (A.6) because $y$ and $Y$ correspond, respectively, to $(x, \dot{x})$ and $(X, \hat{X})$. Differentiating (A.10) with respect to $X$ and $\dot{X}$, we have:

(A.11)

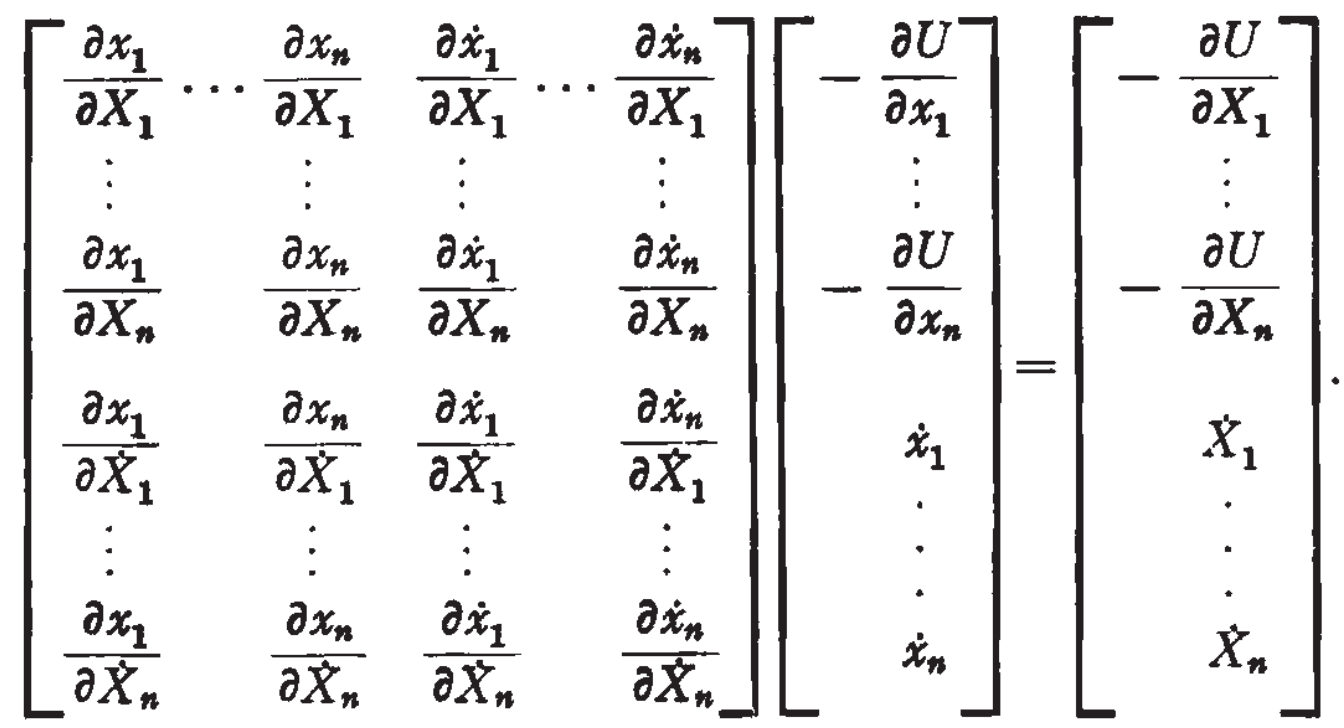

We shall denote by $A(t)$ the $2 n \times 2 n$ matrix on the left-hand side of (A.11), or

$$
A(t) \equiv\left[\begin{array}{ccc}
\frac{\partial x_{1}}{\partial X_{1}} & \cdots & \frac{\partial \dot{x}_{n}}{\partial X_{1}} \\
\vdots & & \vdots \\
\frac{\partial x_{1}}{\partial \dot{X}_{n}} & & \frac{\partial \dot{x}_{n}}{\partial \dot{X}_{n}}
\end{array}\right],
$$

and we shall demonstrate the existence and regularity of $A(t)$, because these are essential for the proof of the central theorem. For instance, the transpose $A$ * may be found from

$$
\frac{d A^{*}(t)}{d t}=J(x) A^{*}(t)
$$

with the initial condition $A^{*}(0)=I$ where $I$ is the identity matrix. Since $J$ is of class $C^{1}$, the existence, uniqueness, and continuity of $A(t)$ is assured. But $J$ is a function of the configuration $x$; hence the solution of (A.13) depends on the initial configuration $X$, and we write it as 


$$
A=A(t, X)
$$

Let us consider $A$ as a mapping in the phase space which, for fixed $t$ and $X$ maps the point $(-\nabla U, \dot{x})$ onto the initial point $\left(-\nabla_{0} U, \dot{X}\right)$ where $\nabla_{0} U$ denotes $\operatorname{grad} U$ at $X$. Then, $A$ has the important property, formulated in

Lemma A-III: For fixed $t$ and $X$, the mapping $A$ is volume-preserving; i.e., the determinant of $A$ is unity. Consequently, a unique inverse of $A$ exists.

To prove this lemma, we make use of the Jacobi-Liouville formula

$$
\operatorname{det}\left|A^{*}(t)\right|=\exp \int_{0}^{t} \operatorname{tr} J(s) d s
$$

where $J$ is defined in (A.5), and $\operatorname{tr} J$ denotes the trace of $J$. Now, the elements in the main diagonal of $J$ are all zero. Therefore, $\operatorname{det} A$ is unity and the inverse $A^{-1}$ exists. Thus, we may write (A.11) as

$$
\left[\begin{array}{c}
-\nabla U \\
\dot{x}
\end{array}\right]=A^{-1}(t)\left[\begin{array}{c}
-\nabla_{0} U \\
\dot{X}
\end{array}\right],
$$

and $A^{-1}$ is a one-to-one mapping in the phase space which maps the point $\left(-\nabla_{0} U, \dot{X}\right)$, for fixed $t$ and $X$, onto a point $(-\nabla U, \dot{x})$.

In the following, we shall only consider trajectories originating from an initial point $P_{0}$ lying on the bounding surface; i.e., at $x=X, \dot{x}=0$. These are called $T$-curves (see Section VII.2); they correspond to motions with a rest-point. We shall now prove

Theorem A-II: Along every T-curve there is a countable infinity of isolated points $Q_{1}, Q_{2}, \ldots$, at which the configuration is one of stationary potential energy with respect to points on $T$ neighboring on $Q_{1}, Q_{2}, \ldots$.

The proof of this theorem is lengthy, and we shall only sketch it. At every $Q_{i},(j=1,2, \ldots)$ one has

$$
\frac{\partial U}{\partial t}=0
$$

and the theorem holds if (A.17) has a countable infinity of isolated $t_{j}$, $(j=1,2, \ldots)$.

First, we note that (A.17) may be rewritten in the form

$$
\sum_{i=1}^{n} \frac{\partial U}{\partial x_{i}} \dot{x}_{i}=\langle\nabla U, \dot{x}\rangle=0
$$


which shows that the gradient $\nabla U$ is orthogonal to the velocity vector $\dot{x}$ at all $Q_{j}$ or, at the $Q_{j}$, the velocity vector lies in a tangent plane (actually the intersection of the tangent surfaces) to an equipotential surface.

Now, $T$-curves are either tangent to equipotential surfaces at finite times $t_{j}$ or, if they are not, they tend to an equipotential surface as $t \rightarrow \infty$. To prove Theorem A-II, we need only show that the latter cannot arise. We suppose that a trajectory (not necessarily a $T$-curve) lies in an equipotential surface. Such a trajectory either is a closed orbit, or it tends asymptotically and monotonically to a closed orbit. This latter is true because the transversals (see VII-1) are, by definition, orthogonal to the equipotential surfaces; thus, no trajectory, lying in an equipotential surface, can have curvature components in a tangent plane (as above) to that surface.

Next, we observe that, if there exists a closed trajectory in an equipotential surface, it must lie in a two-dimensional plane which contains the origin. This is true because such an orbit must be symmetric with respect to the origin by virtue of Theorem V-III. Thus, if a $T$-curve tends to a closed orbit which lies in an equipotential surface it will, after a finite time, have a position and slope which differs from that of the closed orbit by as little as we please. Since that $T$-curve satisfies the system $M$ of (3.12), the closed orbit must satisfy (3.12) within $O(\varepsilon)$. But it can be shown that the only two-dimensional orbit lying in an equipotential surface which satisfies (3.12) is a circle. That demonstration is based on the observation that $\|\dot{x}\|^{2}=$ const for any trajectory in an equipotential surface. Finally, we show that equipotential surfaces of admissible systems do not contain circles in a plane containing the origin, because such equipotential surfaces occur only in systems which are not elastically coupled throughout; i.e., which satisfy (2.4). Thus, no trajectory satisfying (3.12) can be asymptotic to an equipotential surface; consequently, we have proved that $\partial U / \partial t$ vanishes for bounded $t_{j}$. By the same arguments as above, no arc of finite length of a trajectory can coincide with an equipotential surface because, if it did, the arc would have to be that of a circle about the origin, and no equipotential surface contains such arcs. This proves Theorem A-II.

Let us denote by $h_{j}$ the energy levels of the $Q_{j}$, and let us write

$$
t_{j}=t_{j}(X)
$$

because it has been shown that the $t_{j}$ depend on the initial point $X$.

We wish to show that every $h_{j}$ is continuous and differentiable with respect to the initial point $X$. The differential of $U$ is

$$
d U=U_{t} d t+\sum_{\alpha=1}^{n} U_{X_{\alpha}} d X_{\alpha}
$$

for variations in transit time through a given energy level, and for variations in the initial point. The existence of this differential is assured by the prop- 
erties of $U$ and the uniqueness of (A.6). Consider now one of the $h_{j}$, say $h_{r}$. Its differential is the same as (A.20) except that $U_{t}=0$ by definition. Hence,

$$
d h_{r}=\sum_{\alpha, \beta}^{n} \frac{\partial U}{\partial x_{\alpha}} \frac{\partial x_{\alpha}}{\partial X_{\beta}} d X_{\beta}
$$

Thus, the $h_{j}$ are continuous and differentiable with respect to the initial point $P_{0}(X)$.

To prove the existence of a simple trajectory we extremize $h_{j}(X)$ subject to the equation of constraint

$$
U(X)+h=0 .
$$

In other words, we determine the stationary values of the potential energy for a trajectory and we insure by the constraint equation (A.22) that this trajectory is a $T$-curve. Thus, we define a function

$$
g(X)=h_{j}(X)-\lambda U(X)
$$

where $\lambda$ is a Lagrangian multiplier. Since we wish to deal here with the phase space we may write, instead of (A.23)

$$
g(X, X)=h_{j}(X)+O\left(\sum_{i=1}^{n}{\dot{x_{i}}}^{2}\right)-\lambda\left[U(X)+\sum_{i=1}^{n} \dot{X}_{i}^{2}\right]
$$

with $\dot{X}=0$. Then, the necessary conditions for the extremum are

$$
\frac{\partial g}{\partial X_{\alpha}}=0, \quad \frac{\partial g}{\partial \dot{X}_{\alpha}}=0, \quad(\alpha=1, \ldots, n)
$$

and, in view of (A.21), we have at $t=t_{j}$

(A.26)

$$
\left\{\begin{array}{l}
\frac{\partial h_{j}}{\partial X_{\alpha}}=\sum_{\beta=1}^{n} \frac{\partial U}{\partial x_{\beta}} \frac{\partial x_{\beta}}{\partial X_{\alpha}}, \\
\frac{\partial h_{j}}{\partial \dot{X}_{\alpha}}=\sum_{\beta=1}^{n} \frac{\partial U}{\partial x_{\beta}} \frac{\partial x_{\beta}}{\partial \dot{X}_{\alpha}} .
\end{array}\right.
$$

Then, we may rewrite (A.25) as

$$
A\left(t_{j}, X\right)\left[\begin{array}{c}
-\nabla U \\
0
\end{array}\right]=\lambda\left[\begin{array}{c}
\nabla_{0} U \\
0
\end{array}\right]
$$

where $A(t, X)$ has been defined in (A.12). But, if we write (A.16) at $t=t_{i}$ we have

$$
A\left(t_{j}, X\right)\left[\begin{array}{c}
-\nabla U \\
\dot{x}
\end{array}\right]=\left[\begin{array}{c}
-\nabla_{0} U \\
0
\end{array}\right]
$$


or, combining the last two,

$$
A\left(t_{j}, X\right)\left[\begin{array}{cc}
(\lambda-1) & \nabla U \\
-\lambda & \dot{x}
\end{array}\right]=0 .
$$

But, since $\operatorname{det} A=1$, the only possible solutions to (A.27) are

$$
\lambda=0 \quad \text { and } \quad \nabla U=0 \text {; }
$$

$$
\lambda=1 \quad \text { and } \quad \dot{x}=0 \text {. }
$$

The first of these states that $U_{t}=0$ at the origin since $\nabla U=0$ at the origin only, and the second, that $U_{t}=0$ on the bounding surface because that surface is defined by $\dot{x}=0$. Hence, a $T$-curve $T^{*}$ exists which connects an initial point on the bounding surface with the origin. To prove Theorem A-I, it remains to show that the $T^{*}$-curve is a simple trajectory.

Let $P_{0}^{*}$ be the initial point of $T^{*}$ on the bounding surface. Let its points of first, second, etc. tangency with equipotential surfaces be $Q_{1}, Q_{2}, \ldots$.

Now, suppose that $Q_{1}$ is not at the origin. Then, in view of the above demonstration, $Q_{1}$ is on the bounding surface. In that case, $T^{*}$ moves at first toward configurations of lower potential energy and then toward configurations of higher potential energy. Thus, there is a point $R$ between $P_{0}{ }^{*}$ and $Q_{1}$ at which $U_{t}=0$. But this contradicts the fact that $Q_{1}$ is the first point along $T^{*}$ where $U_{t}=0$. Hence, $Q_{1}$ is at the origin. This completes the proof.

\section{ACKNOWLEDGMENT}

This work was supported, in part, by a grant from the National Science Foundation.

\section{Reterences}

1. Minorski, N., "Nonlinear Oscillations," Van Nostrand, N. J., 1962.

2. Synge, On the geometry of dynamics, Phil. Trans. A, 226, 31-106 (1926).

3. Rauschrr, M., Steady oscillations of systems with nonlinear and unsymmetrical elasticity, Journ. App. Mech. 5, A169-A177 (1938).

4. Stoker, J. J., "Nonlinear Vibrations," Interscience Publ., New York, 1950.

5. Ledoux, P., Personal communication, Liège, 1964.

6. Mnwhin, J., Oscillations en modes normaux de systèmes dynamiques nonlinéaires à plusieurs degrés de liberté, Bull. Soc. Royale Sciences Liége, 9-10, 540-557 (1964).

7. Rosenberg, R. M., On normal mode vibrations, Proc. Camb. Phil. Soc., 60, 595-611 (1964). 
8. Rosenberg, R. M. and KUo, J, K., Nonsimilar normal mode vibrations of nonlinear systems having two degrees of freedom, Trans. ASME, Journ. Appl. Mech., 81, E, 2, 283-290 (1964).

9. RosenberG, R. M. and Hsu, C. S., On the geometrization of normal vibrations of nonlinear systems having many degrees of freedom, in Proc. IUTAM Symp. on Nonl. Vibr., Ukrainian Academy of Sciences, Kiev, 1, 380-415 (1963).

10. KaUderer, H., Nichtlineare Mechanik, Springer, Göttingen, 1958.

11. Rosenberg, R. M., The normal modes of nonlinear n-degree-of-freedom systems, Trans. ASME, Journ. Appl. Mech., 29, E, 1, 7-14 (1962).

12. KINNEY, W. D., On the geometrization of the forced oscillations of nonlinear systems having many degrees of freedom, Ph. D. Thesis, Univ. of Calif., Berkeley, 1905.

13. KINNEy, W. D. and Rosenberg, R. M., On steady-state forced vibrations in strongly nonlinear systems having two degrees of freedom (Les vibrations forcées dans les systèmes nonlinéaires), Proc. CNRS Int. Coll. 148, 351-373 (1965).

14. DarbouX, G., "Théorie Générale des Surfaces," vol. 2, pp. 438-509, Gauthier-Villar, Paris, 1889.

15. HaAG, J., "Oscillatory Motions" (Translation of "Les Mouvements Vibratoires"), vol. 1, pp. 35-39, Wadsworth Publ. Co., 1962.

16. Rosenberg, R. M., La géometrie de la dynamique et les vibrations des systèmes non-linéaires, L'Onde Electrique 48, 433, 425-434 (1963).

17. Courant, R. and Hilbert, D., "Methoden der mathematischen Physik“, vol. 1, pp. 240-245, Springer, Berlin, 1931.

18. KUo, J. K., On non-similar normal mode vibrations of nonlinear systems having many degrees of freedom, Ph. D. Thesis, Univ. of Calif., Berkeley, 1964.

19. RosenberG, R. M., On the existence of normal mode vibrations of nonlinear systems with two degrees of freedom, Quart. Appl. Math., 22, 3, 217-234 (1964).

20. Rosenberg, R. M., The ateb(h)-functions and their properties, Quart. Appl. Math. Q1, 6, 37-47 (1963).

21. Rosenberg, R. M., "Les vibrations non-linéaires de systèmes à plusieurs degrés de liberté," Centre de Rech. Phys., Marseille, 1964.

22. Sideriades, L. and Argemi, J., Sur la singularité multiple des systèmes dynamiques plans, Comp. Rend., Acad. Scie. 253, 2037-2039, Gauthier-Villar, Paris (1961).

23. Argemi, J. and Sideriadés, L., Sur la singularité multiple définie par les formes homogènes des systèmes dynamiques plans, Comp. Rend. 254, 4135-4136. Paris (1962).

24. Rosenberg, R. M., On motions with a rest point and the existence of normal mode vibrations in nonlinear systems, in "Proc. Eleventh. Int. Congr. Appl. Mech., Munich" 1964, (to appear).

25. Maharem, N., On homogeneous systems and the stability of their normal modes, Ph. D. Thesis, Univ, of Calif., Berkeley, 1965.

26. Codpington, E. A. and Levinson, N., "Theory of Ordinary Differential Equations," McGraw-Hill, New York, 1955.

27. HaUghton, K. E., Similar motion of multi-degree-of-freedom vibrating systems with nonsymmetric springs, $\mathrm{Ph}$. D. Thesis, Univ. of Calif., Berkeley, 1964.

28. Rosenberg, R. M., On normal vibrations of a general class of nonlinear dual-mode systems, Trans. ASME, Journ. Appl. Mech., 28, E, 2, 275-283 (1961).

29. Rosenberg, R. M. and Atkinson, C. P., On the natural modes and their stability in nonlinear two-degree-of-freedom systems, Trans. ASME, Journ. Appl. Mech., 26, E, 3, 377-385 (1959). 
30. Whittaker, E. T. and Watson, G. N., "Modern Analysis," Cambridge Univ. Press, London and New York, p. 524, 1927.

31. Misshkes, A. D., On the exactness of approximate methods of analyzing small nonlinear vibrations of one degree of freedom, in "Dynamic Problems" (coll. papers) vol. 1, p. 139, publishers, Riga, 1953 (in Russian).

32. McDufF, J. N. and CuRreri, J. R., "Vibration Control," McGraw-Hill, New York, p. $72,1958$.

33. Landau, L. D. and Lifshitz, E. M., "Mechanics," Pergamon Press and AddisonWesley, London, Reading, Mass., p. 27, 1960.

34. Pearson, K., "Tables of Incomplete Beta Functions," Cambridge Univ. Press, London and New York, 1927.

35. Rosenberg, R. M., On a geometrical method in nonlinear vibrations (Les vibrations forcées dans les systèmes non-linéaires), Proc. CNRS Int. Coll., 148, 69-79 (1965).

36. Trmoshenko, S., "Vibration Problems in Engineering," Van Nostrand, Princeton, N. J., 1955.

37. Den Hartog, J. P., "Mechanical Vibrations," Third ed., McGraw-Hill, New York, 1947.

38. KLOTtER, K., Steady-state vibrations in systems having arbitrary restoring and arbitrary damping forces, in "Proc. Symp. Nonl. Circuit Anal. 234-257, Poly. Inst. Brooklyn" (1953).

39. Struble, R. A., "Nonlinear Differential Equations," McGraw-Hill, New York, 1962.

40. Hsu, C. S., On a restricted class of coupled Hill's equations and some applications, Trans. ASME, Journ. Appl. Mech., 28, E, 4, 551-556 (1961).

41. Lovide, W., Personal communication (1961).

42. Maclachlan, N. W., "Ordinary Non-Linear Differential Equations in Engineering and Physical Sciences," Clarendon Press, Oxford, 1950.

43. Hsu, C. S., On the parametric excitation of a dynamic system having multiple degrees of freedom, Trans. ASME, Journ. Appl. Mech., 80, E, 3, 367-372, (1963).

44. Hsu, C. S., On the stability of periodic solutions of nonlinear dynamical systems under forcing, Proc. CNRS Int. Coll., (Les vibrations forcées dans les systemes non-linéaires), 148, 125-141 (1965). 